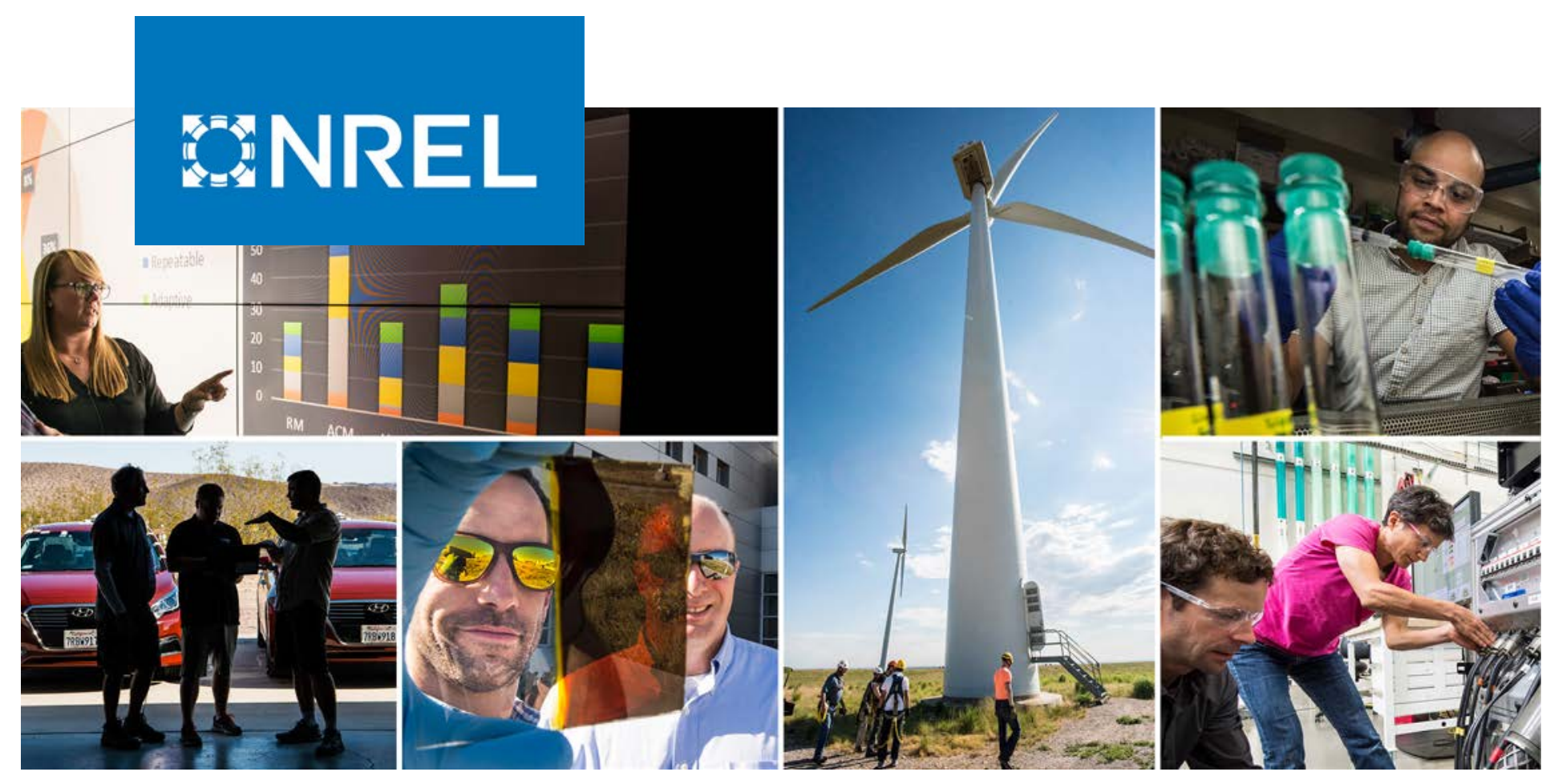

\title{
Big Adaptive Rotor Phase I Final Report
}

Nick Johnson, ${ }^{1}$ Josh Paquette, ${ }^{2}$ Pietro Bortolotti, ${ }^{1}$

Nicole Mendoza, ${ }^{1}$ Mark Bolinger, ${ }^{3}$ Ernesto Camarena, ${ }^{2}$

Evan Anderson, ${ }^{2}$ and Brandon Ennis ${ }^{2}$

1 National Renewable Energy Laboratory

2 Sandia National Laboratories

3 Lawrence Berkeley National Laboratory

NREL is a national laboratory of the U.S. Department of Energy

Office of Energy Efficiency \& Renewable Energy

Operated by the Alliance for Sustainable Energy, LLC

This report is available at no cost from the National Renewable Energy Laboratory (NREL) at www.nrel.gov/publications.

\section{Technical Report}

NREL/TP-5000-79855

December 2021 


\section{GNREL}

\section{Big Adaptive Rotor Phase I Final Report}

Nick Johnson, ${ }^{1}$ Josh Paquette ${ }^{2}$ Pietro Bortolotti, ${ }^{1}$

Nicole Mendoza, ${ }^{1}$ Mark Bolinger, ${ }^{3}$ Ernesto Camarena, ${ }^{2}$

Evan Anderson, ${ }^{2}$ and Brandon Ennis ${ }^{2}$

1 National Renewable Energy Laboratory

2 Sandia National Laboratories

3 Lawrence Berkeley National Laboratory

\section{Suggested Citation}

Johnson, Nick, Josh Paquette, Pietro Bortolotti, Nicola Mendoza, Mark Bolinger, Ernesto Camarena, Evan Anderson, and Brandon Ennis. 2021. Big Adaptive Rotor Phase I Final Report. Golden, CO: National Renewable Energy Laboratory. NREL/TP-5000-79855.

https://www.nrel.gov/docs/fy220sti/79855.pdf.

NREL is a national laboratory of the U.S. Department of Energy Office of Energy Efficiency \& Renewable Energy Operated by the Alliance for Sustainable Energy, LLC

This report is available at no cost from the National Renewable Energy Laboratory (NREL) at www.nrel.gov/publications.

Contract No. DE-AC36-08GO28308
Technical Report

NREL/TP-5000-79855

December 2021

National Renewable Energy Laboratory 15013 Denver West Parkway Golden, CO 80401

303-275-3000 • www.nrel.gov 


\section{NOTICE}

This work was authored in part by the National Renewable Energy Laboratory, operated by Alliance for Sustainable Energy, LLC, for the U.S. Department of Energy (DOE) under Contract No. DE-AC36-08GO28308. Funding provided by the U.S. Department of Energy Office of Energy Efficiency and Renewable Energy Wind Energy Technologies Office. The views expressed herein do not necessarily represent the views of the DOE or the U.S. Government.

This report is available at no cost from the National Renewable Energy Laboratory (NREL) at www.nrel.gov/publications.

U.S. Department of Energy (DOE) reports produced after 1991 and a growing number of pre-1991 documents are available free via www.OSTI.gov.

Cover Photos by Dennis Schroeder: (clockwise, left to right) NREL 51934, NREL 45897, NREL 42160, NREL 45891, NREL 48097, NREL 46526.

NREL prints on paper that contains recycled content. 


\section{Acknowledgments}

The National Renewable Energy Laboratory acknowledges the U.S. Department of Energy Wind Energy Technologies Office for supporting this work under the Office of Energy Efficiency and Renewable Energy's technology research and development activities. Sandia National

Laboratories, Oak Ridge National Laboratory, and Lawrence Berkeley National Laboratory were partners on this document. Sandia National Laboratories is a multimission laboratory managed and operated by National Technology \& Engineering Solutions of Sandia, LLC, a wholly owned subsidiary of Honeywell International Inc., for the U.S. Department of Energy's National Nuclear Security Administration under contract DE-NA0003525 


\section{List of Acronyms}

\begin{tabular}{|c|c|}
\hline AEP & annual energy production \\
\hline ANBA & ANisotropic Beam Analysis \\
\hline BAR & Big Adaptive Rotor \\
\hline BAR-DRC & BAR - downwind - rail transport - carbon-fiber spar caps \\
\hline BAR-DRG & BAR - downwind - rail transport - glass-fiber spar caps \\
\hline BAR-UAG & BAR - upwind - air transport - glass-fiber spar caps \\
\hline BAR-URC & BAR - upwind - rail transport - carbon-fiber spar caps \\
\hline BAR-USC & BAR - upwind - segmented - carbon-fiber spar caps \\
\hline BEM & blade element momentum \\
\hline BOS & balance of system \\
\hline CAISO & California Independent System Operator \\
\hline CapEx & capital expenditure \\
\hline CART2 & Controls Advanced Research Turbine \\
\hline $\mathrm{CF}$ & capacity factor \\
\hline CFD & computational fluid dynamics \\
\hline $\mathrm{DAC}$ & distributed aerodynamic control \\
\hline DOE & U.S. Department of Energy \\
\hline ERCOT & Electric Reliability Council of Texas \\
\hline FEM & finite element model \\
\hline FSI & fluid-structure interaction \\
\hline IEA & International Energy Agency \\
\hline IEC & International Electrotechnical Commission \\
\hline ISO & Independent System Operator \\
\hline $\mathrm{kW}$ & kilowatt \\
\hline LBNL & Lawrence Berkeley National Laboratory \\
\hline $\mathrm{LCOE}$ & levelized cost of energy \\
\hline LTA & lighter than air \\
\hline LWST & low-wind-speed turbine \\
\hline $\mathrm{M}$ & meter \\
\hline MISO & Midcontinent Independent System Operator \\
\hline MW & megawatt \\
\hline MWh & megawatt-hour \\
\hline MWST & moderate-wind-speed turbine \\
\hline NREL & National Renewable Energy Laboratory \\
\hline NuMAD & Numerical Manufacturing And Design Tool \\
\hline NYISO & New York Independent System Operator \\
\hline O\&M & operation and maintenance \\
\hline OLAF & cOnvecting LAgrangian Filaments \\
\hline PIRT & phenomena identification and ranking table \\
\hline PJM & Pennsylvania, Jersey, Maryland Power Pool \\
\hline PPA & power purchase agreement \\
\hline $\mathrm{R} \& \mathrm{D}$ & research and development \\
\hline $\mathrm{ROSCO}$ & Reference OpenSource Controller \\
\hline Sandia & Sandia National Laboratories \\
\hline SONATA & Structural Optimization aNd AeroelasTic Analysis \\
\hline
\end{tabular}


SP

SPP

SwiFT

TCC

TRL

VABS

WEIS

WISDEM specific power

Southwest Power Pool

Scaled Wind Farm Technology

turbine capital cost

technology readiness level

Variational Asymptotic Beam Sectional Analysis

Wind Energy with Integrated Servo-control

Wind-Plant Integrated System Design \& Engineering Model 


\section{Executive Summary}

The Big Adaptive Rotor (BAR) project was initiated by the U.S. Department of Energy (DOE) in 2018 with the goal of identifying novel technologies that can enable large $(>100$ meter $[\mathrm{m}])$ blades for low-specific-power wind turbines. Five distinct tasks were completed to achieve this goal:

1. Assessed the trends, impacts, and value of low-specific-power wind turbines

2. Developed a wind turbine blade cost-reduction road map study

3. Completed research-and-development opportunity screening

4. Performed detailed design and analysis

5. Assessed low-cost carbon fiber.

These tasks were completed by the national laboratory team consisting of Sandia National Laboratories (Sandia), the National Renewable Energy Laboratory (NREL), and Lawrence Berkeley National Laboratory. The objectives of Task 1 were to assess the historical trends of low-specific-power land-based deployments and to assess the impact and value of low-specificpower wind turbines. Lawrence Berkeley National Laboratory and NREL produced two publications on these topics and showed that there is a distinct trend toward low-specific-power turbines and that their presence coincides with reduced levelized cost of energy (LCOE). Scenarios for which large low-specific-power turbines are the preferred configuration for landbased deployments were also detailed under this task, and a compelling case was made that continued growth and lower specific-power configurations could be market leaders in the future. Additionally, Task 1 conducted analysis on the market value of low-specific-power wind turbines beyond the typical LCOE metric. The analysis showed that there is significant value for low-specific-power turbines, especially in markets where there is a higher saturation of wind energy on the electric grid. This is because low-specific-power turbines have increased capacity factors and can contribute more reliable energy to the grid, even in lower wind conditions. This bodes well, as higher penetration renewable scenarios are likely in the coming years.

Task 2 was a detailed analysis on the logistical challenges of deploying very large $(>100 \mathrm{~m})$ land-based blades. This work was completed by experts at DNV GL. The report looked at various ways to manage the current transportation constraints, which are estimated to be approximately $75 \mathrm{~m}$ in length. The report concluded that there are viable solutions to this issue, including segmented blades (which the industry is already pursuing), airships, on-site manufacturing, and controlled bending of blades on rail. The final option was recommended for further analysis and study by DOE and the national labs because keeping a single-piece blade reduces manufacturing and operation-and-maintenance costs. The challenge is to design a blade that considers the flexibility requisite to navigate curvatures in the rail line without damaging either the blade or the rail. To that end, NREL conducted a detailed study on the necessary flexibility and eventual operational loads of a 100-m blade capable of navigating common curvatures on rail lines. The findings showed that it is possible to transport and operate such a blade. A downwind configuration was considered in this task because of the reduced tip-to-tower clearance restrictions as compared to upwind configurations.

Task 3 focused on identifying novel concepts that can enable a cost-effective 5-megawatt wind turbines with a 206-m rotor for land-based deployment. The findings from the first two tasks were considered in the analysis in that the concepts identified must be able to overcome the 
transportation logistic challenges. Nearly 20 concepts were identified and evaluated by experts within the industry. Additionally, science and engineering challenges were identified for each concept. Based on these evaluations, six concepts were deemed the most impactful and were selected for further analysis in Task 4: highly flexible rail-transportable blades, downwind rotors, distributed aerodynamic control devices, inflatable blades, bi-wing blades, and five-bladed rotors.

Task 4 conducted detailed design, optimization, and analysis on the selected BAR concepts. A modeling gaps analysis was conducted, and modeling improvements were implemented that allowed for the study and design of the novel concepts. The BAR team established a set of baseline designs by which to compare the selected designs through a techno-economic analysis. It was found that the highly flexible rail-transportable blade, the downwind rotor, and the distributed aerodynamic control devices have the most promise to deliver the BAR targets. A Phase II for BAR has been proposed to further mature these concepts and to address the open science and engineering challenges identified in Task 3.

Task 5 conducted research on optimized carbon-fiber materials that were used throughout the BAR Phase I project. This task produced and characterized textile carbon-fiber specimens. Cost reductions of $56 \%$ were achieved as compared to the industry baseline carbon fiber. These advanced materials reduce the LCOE of large wind turbines.

Overall, the BAR project has identified low-specific-power turbine designs as important to continued LCOE reductions for land-based wind turbines. Further, the project has identified viable solutions to the technical and logistical challenges to realizing these goals. The most promising technologies that were identified in Phase I of the project will be further matured and derisked in Phase II of the BAR project. 


\section{Table of Contents}

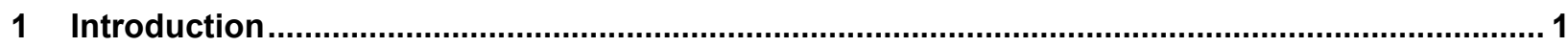

2 Task 1: Assess Trends, Impacts, and Value of Low-Specific-Power Wind Turbines................... 3

2.1 Subtask 1.A: Trends Analysis for Lower-Specific-Power Wind Turbines ............................... 3

2.2 Subtask 1.B: Value of Low-Specific-Power Wind Turbines and High-Performance Wind Power

Plants

\section{1}

2.3 Subtask 1.C: National Geospatial and Market Impacts of Low-Specific-Power Turbines and High-Performance Wind Power Plants

3 Task 2: Wind Turbine Blade Cost Reduction Road Map Study .................................................. 22

4 Task 3: Research-and-Development Opportunity Screening …….............................................. 35

4.1 Subtask 3.A: Development of Big Adaptive Rotor Innovation Concept Catalog ...................... 35

4.2 Subtask 3.B: Information Gathering To Support Techno-Economic Analysis and Identification

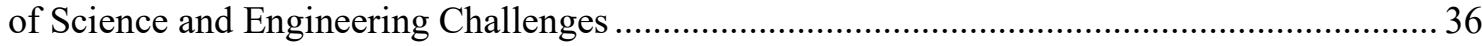

4.3 Subtask 3.C: Big Adaptive Rotor Innovation Concept Initial Techno-Economic Analysis and Investigation of Science and Engineering Challenges ............................................................. 39

$5 \quad$ Task 4: Detailed Design Studies ..................................................................................... 42

5.1 Subtask 4.A: Identification of Science and Engineering Challenges and Modeling Gaps.......... 42

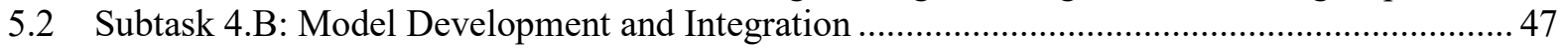

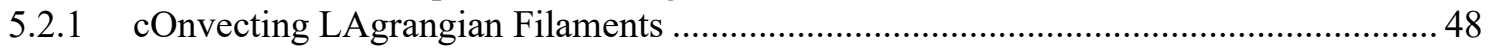

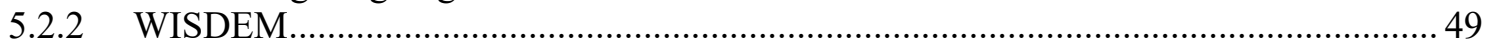

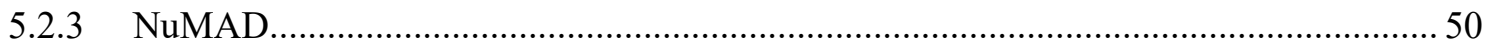

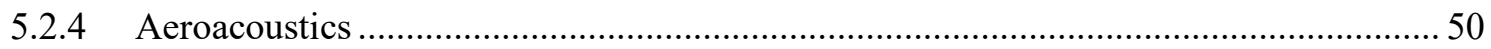

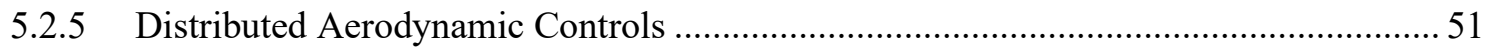

5.3 Subtask 4.C: Detailed Design for Big Adaptive Rotor Innovation Concepts .......................... 51

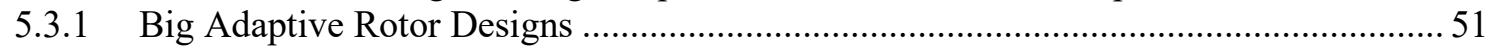

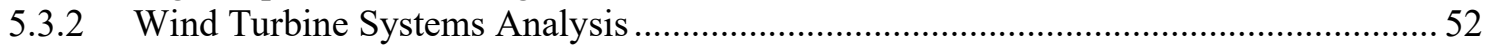

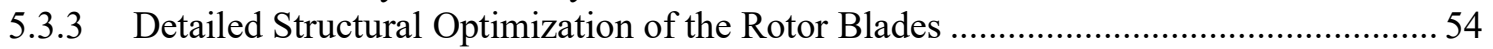

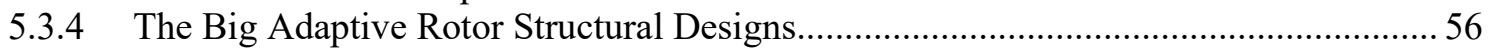

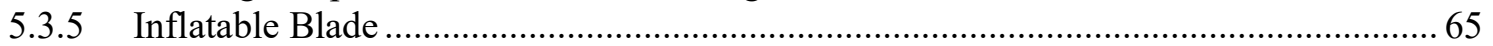

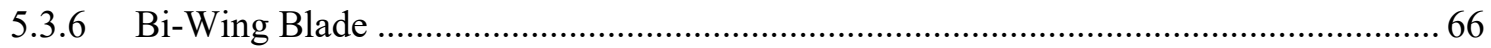

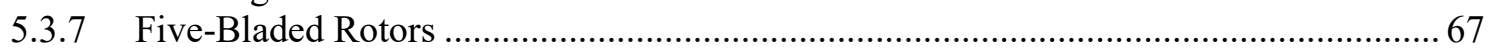

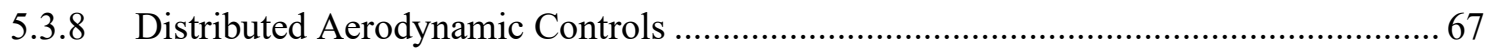

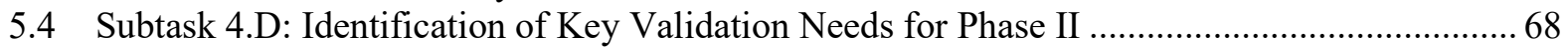

6 Task 5: Optimized Carbon Fiber for Wind Turbines …............................................................... 72

6.1 Subtask 5.A: Blade and Material Cost Model Development ................................................... 72

6.2 Subtask 5.A: Blade and Material Cost Model Development ................................................... 73

6.3 Subtask 5.B: Rotor Cost Optimization Studies ........................................................................ 74

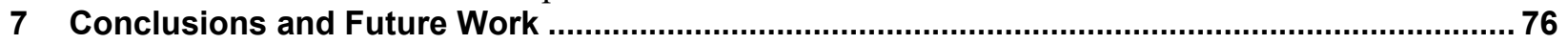

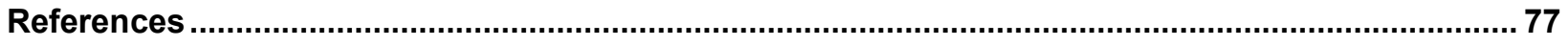

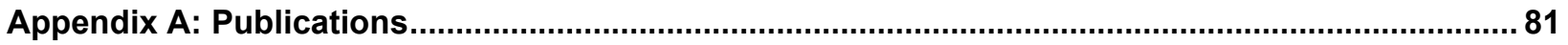




\section{List of Figures}

Figure 1. Trends in wind turbine capacity (MW), hub height $(\mathrm{HH})$, rotor diameter (RD), and specific

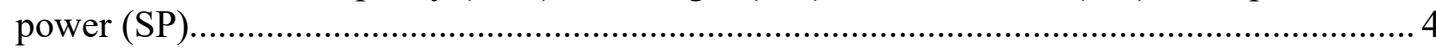

Figure 2. Cumulative percentage change in key turbine parameters since 2009 .................................. 5

Figure 3. Map of wind project location and specific power overlaid on long-term average wind speed as estimated based on typical meteorological year conditions for the period of $1997-2010$...... 6

Figure 4. Capacity factor in 2018 as a function of long-term average wind speed and specific power........ 8

Figure 5. Capacity factor in 2018 as a function of binned wind speed and specific power $(\mathrm{GW}=$

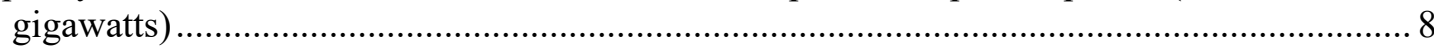

Figure 6. Average specific power and capacity factor by project vintage ............................................. 9

Figure 7. Mass intensity of Vestas 2.0-MW wind turbines as a function of specific power (Sources: Garrett and Rønde 2011a, 2011b; Razdan and Garrett 2018a, 2018b, 2015a, 2015b).......... 10

Figure 8. Trends in PPA prices and LCOE by project vintage (bubble size corresponds to PPA capacity)

Figure 9. Percentage change in 2018 market value from low-specific-power, tall turbine vs. 2018 average turbine

Figure 10. Absolute change in 2018 market value from low-specific-power, tall turbine vs. 2018 average turbine

Figure 11. Average percentage change in value from low-specific-power, tall turbine is correlated with

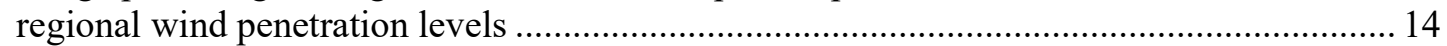

Figure 12. Transmission, balancing, and financing cost savings from low specific-power, tall turbines... 15

Figure 13. Net capacity factor distributions for each of the four turbines analyzed ................................ 18

Figure 14. Net capacity factor of Low SP and High SP turbines relative to the Constant SP turbine........ 18

Figure 15. Relative change in CapEx value that would result in an equivalent LCOE as the Constant SP turbine

Figure 16. Hypothetical configuration of the manufacturing facilities and project locations..................... 25

Figure 17. Calculated total cost of delivered blade targets ................................................................ 27

Figure 18. Innovation pathway summary - total cost of delivered blades, Michigan ..............................29

Figure 19. Innovation pathway summary - total cost of delivered blades, Montana ...............................2 29

Figure 20. Innovation pathway summary - impact on system LCOE, Michigan ..................................... 30

Figure 21. Innovation pathway summary - impact on system LCOE, Montana ....................................30

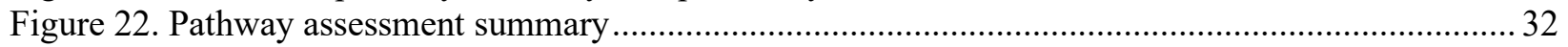

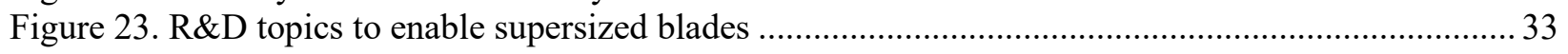

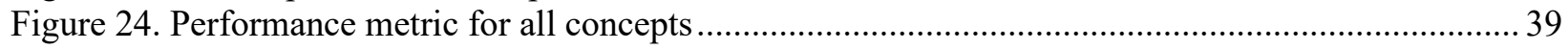

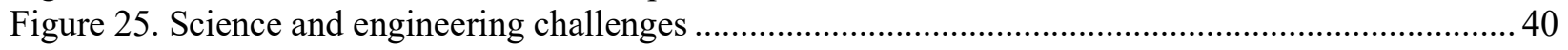

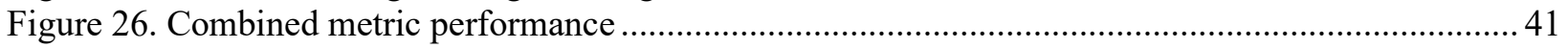

Figure 27. Evolution of near-wake lattice, blade-tip vortex, and Lagrangian markers............................ 49

Figure 28. The design workflow adopted to develop the flexible rail-transportable BAR rotors.............. 53

Figure 29. Coordinates of the airfoils used in the design of the BAR flexible blades .............................. 57

Figure 30. Polars of the airfoils used in the design of the BAR flexible blades ....................................... 57

Figure 31. Chord, twist, relative thickness, and prebend distributions of the five BAR designs................58

Figure 32. Spanwise thickness distribution of shell skins for optimized BAR designs........................... 59

Figure 33. Spanwise thickness distribution of spar caps for optimized BAR designs ..............................59

Figure 34. Spanwise thickness distribution of leading- and trailing-edge reinforcement for optimized BAR

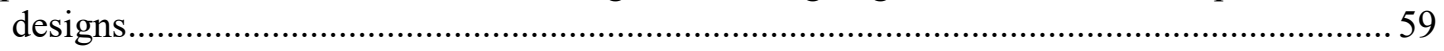

Figure 35. Spanwise thickness distribution of core filler for optimized BAR designs .............................. 60

Figure 36. Spanwise thickness distribution of shear web filler for optimized BAR designs...................... 60

Figure 37. Spanwise thickness distribution of web skins for optimized BAR designs ............................. 61

Figure 38. Tsai-Wu failure index under loading for BAR-DRG from the (a) suction side, (b) pressure side, and (c) trailing edge (cutaway view). The vulnerable point is circled in red. 
Figure 39. Tsai-Wu failure index under loading for BAR-DRC from the (a) suction side, (b) pressure side, and (c) trailing edge (cutaway view). Vulnerable points are circled in red.

Figure 40. Tsai-Wu failure index under loading for BAR-URC from the (a) suction side, (b) pressure side, and (c) trailing edge (cutaway view). Vulnerable points are circled in red. ............................ 63

Figure 41. Displacement plot of most critical buckling mode under loading for BAR-DRG from the (a) suction side and (b) trailing edge

Figure 42. Displacement plot of most critical buckling mode under loading for BAR-DRC from the (a) suction side and (b) trailing edge

Figure 43. Displacement plot of most critical buckling mode under loading for BAR-URC from the (a)

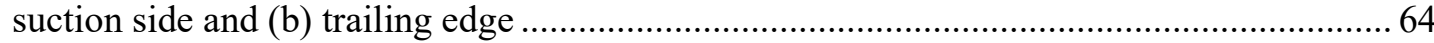

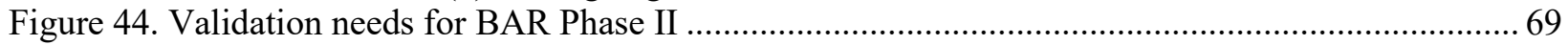

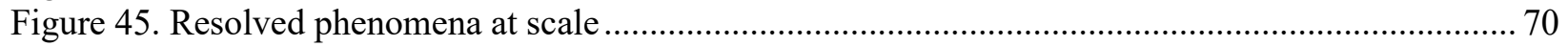

Figure 46. Carbon-fiber catalog tow properties representing a wide range of available commercial

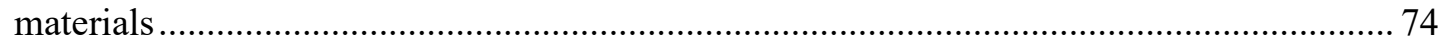

Figure 47. Design studies to be performed in Task 5 to assess the value of carbon fiber beyond the blade

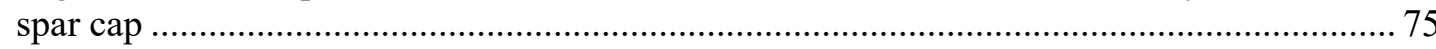

\section{List of Tables}

Table 1. Turbine Configurations Applied in National Supply Curve Modeling...................................... 17

Table 2. Primary Rotor/Turbine Configuration Parameters.................................................................... 26

Table 3. Summary Weight and Cost for Off-Site Monolithic and Modular Blades .................................. 26

Table 4. BAR Concepts Broken Down by Innovation Category …........................................................ 35

Table 5. Cost and Performance Metrics for BAR Concept Evaluation .................................................. 37

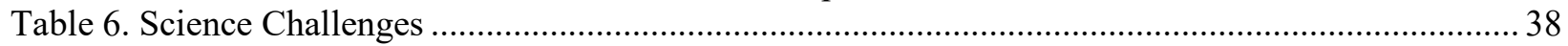

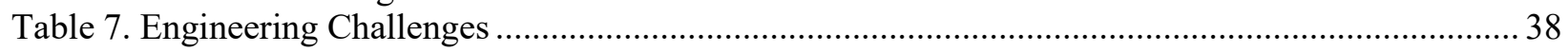

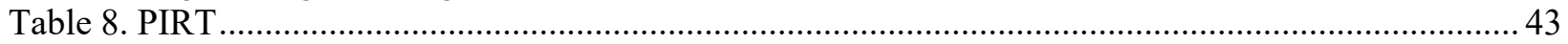

Table 9. Mechanical Properties of Composite Laminates by Fiber Type. All Strength Properties Are

Design Values (Not the Characteristic Values) and Are Obtained With $\gamma \boldsymbol{\sigma}=1.74$............... 55

Table 10. Mechanical Properties of the Isotropic Constituents. All Strength Properties Are Design Values

(Not the Characteristic Values) and Are Obtained With $\boldsymbol{\gamma} \boldsymbol{\sigma}=1.92$ (A larger $\boldsymbol{\gamma} \boldsymbol{\sigma}$ is required by

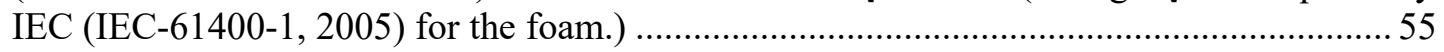

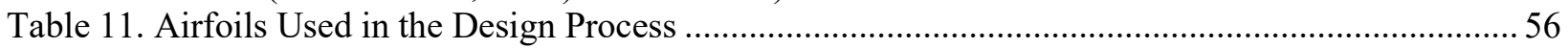

Table 12. Summary of Driving Constraints for Different Blade Designs in Structural Optimization........ 61

Table 13. Summary of the Design Characteristics of the BAR Flexible Rotors....................................... 64

Table 14. Comparison of Total Cost Between Blades Adopting Industry-Standard Unidirectional Carbon

Composite Spar Caps vs. Heavy-Tow Unidirectional Carbon Composite Spar Caps . 65 


\section{Introduction}

Wind turbine and rotor scaling have been the main drivers for levelized cost of energy (LCOE) reductions in wind energy during the past decade (Bolinger et al. 2020). Larger rotors capture substantially more energy through a larger swept area and access to increased wind speeds at higher elevation above the ground. Increased rotor size has led to wind power plants with higher capacity factors and less variability in power production. Further, high-capacity-factor turbines add value to the market in areas with high penetrations of wind, such as the Electric Reliability Council of Texas (ERCOT) region (Wiser 2020). As wind continues to account for more of the electricity generation profile in the United States, this trend is likely to become even more important. Additionally, the nationwide deployment of wind energy that includes lower wind speed sites will require further increases in rotor size and other innovative design concepts for cost-effective energy production; however, there are logistical limits to the size of blades that can be transported via roads and lower-cost rail lines. Current projections are that blades larger than 75 meters $(\mathrm{m})$ in length, $4.75 \mathrm{~m}$ in chord, and $4.5 \mathrm{~m}$ in root diameter are not transportable via traditional methods. As a result, the rotor size of land-based wind turbines is not increasing as quickly as those of offshore machines. Segmentation has been implemented as a partial solution to this problem, though at the cost of added blade mass and potential reliability and maintenance concerns. Segmentation alone, however, does not solve other important challenges for large rotors. For example, joints add weight and reliability concerns. Larger land-based turbines are possible and promise reduced LCOE and access to lower wind resource areas but not without significant technological and logistical innovations.

The goal of the Big Adaptive Rotor (BAR) program is to identify and advance innovative concepts that enable the continued growth of land-based rotors and the continued reduction of specific power (SP) for large turbines $(>5 \mathrm{MW})$ while reducing LCOE. Where SP is defined as the ratio between the turbine rating and rotor swept area. The BAR project was formed as a multilaboratory consortium ${ }^{1}$ in 2018 . Phase I of the project focused on identifying technology to overcome the transportation and operational limitations for a 5-MW wind turbine with a 206-m rotor and developed promising designs incorporating lightweight and flexible structures that are less expensive and more reliable than current segmented-blade approaches. The BAR team investigated several innovative 100-m blade technologies (Johnson et al. 2019), and the most promising BAR concepts were found to be (1) highly flexible, controlled bending blades for rail transport, (2) downwind rotors to accommodate the highly flexible blades without the risk of a tower strike, and (3) distributed aerodynamic control (DAC) devices to control the load on the highly flexible blades. In Phase I, the team also identified several science and engineering challenges and areas of focus to accelerate the advancement of the BAR concepts.

This report contains a summary of the activities completed in Phase I of the BAR project. Five distinct tasks were completed in this project:

1. Assessed the trends, impacts, and value of low-specific-power wind turbines

2. Developed a wind turbine blade cost-reduction road map study

\footnotetext{
${ }^{1}$ The consortium includes Sandia National Laboratories, the National Renewable Energy Laboratory, Lawrence Berkley National Laboratory, and Oak Ridge National Laboratory.
} 
3. Completed research-and-development opportunity screening

4. Performed model improvements and detailed design studies

5. Assessed low-cost carbon fiber.

The remainder of this report focuses on the findings from each task and includes excerpts and citations from previously published work. 


\section{Task 1: Assess Trends, Impacts, and Value of Low- Specific-Power Wind Turbines}

Task 1 was designed to motivate and inform the work happening in parallel under tasks $2-5$ and had three main objectives:

1. To demonstrate and communicate the potential value of the BAR project to a broad stakeholder audience by compiling data and conducting analysis on the historical deployment trends and impacts of relatively low-specific-power wind turbines in the United States

2. To understand the impacts of achieving the targets of the BAR initiative in terms of wind power plant performance, costs, market value, and benefits, recognizing that lowspecific-power turbines have electric sector benefits that go beyond capacity factor and LCOE

3. To help inform and clarify BAR initiative research and development (R\&D) targets and to help prioritize specific technology and R\&D pathways when those pathways have different impacts not only on LCOE but also on wholesale electricity market value and grid integration.

As described in the following subsections, three distinct subtasks were established to achieve these objectives (though in practice there was cross-fertilization and collaboration among subtasks - e.g., Lawrence Berkeley National Laboratory (LBNL) and the National Renewable Energy Laboratory (NREL) jointly published a journal article that covered work conducted under both Subtask 1.A and Subtask 1.C, and the journal article deliverable for Subtask 1.B drew upon content arising from Subtask 1.A).

\subsection{Subtask 1.A: Trends Analysis for Lower-Specific-Power Wind Turbines}

This task provided an early opportunity to compile market data on lower-specific-power wind turbine deployment to date-including when and where such machines have been deployed and their impact on project-level capacity factors. Data and analysis generated under Task 1.A populated numerous conference and panel presentations and formed the basis for the first half of a journal article published jointly between LBNL and NREL covering tasks 1.A and 1.C (Bolinger et al. 2020). 
Following is an excerpt ${ }^{2}$ from that Wind Engineering journal article focusing specifically on the section titled, "Historical Trends, Drivers, and Impacts of Low-Specific-Power Turbines in the United States"

This section documents the historical trend toward the deployment of lowerspecific-power turbines in the United States, as well as the drivers and impacts of this trend. Figure 1 shows the annual averages and distributions of nameplate capacity, hub height, rotor diameter, and specific power of utility-scale (i.e. $>100$ kilowatts $[\mathrm{kW}]$ ) wind turbines installed in the United States over the past decade. The trend toward greater capacity turbines with larger rotors and lower-specificpower ratings is clear: the average nameplate capacity increased from $1.74 \mathrm{MW}$ in 2009 to $2.43 \mathrm{MW}$ in 2018, while the average rotor diameter grew from 81.5 to 115.6 meters $(\mathrm{m})$ (pushing the average swept area from $5,200 \mathrm{~m}^{2}$ to $10,500 \mathrm{~m}^{2}$ ), reducing the average specific power rating from $329 \mathrm{~W} / \mathrm{m}^{2}$ to $230 \mathrm{~W} / \mathrm{m}^{2}$.
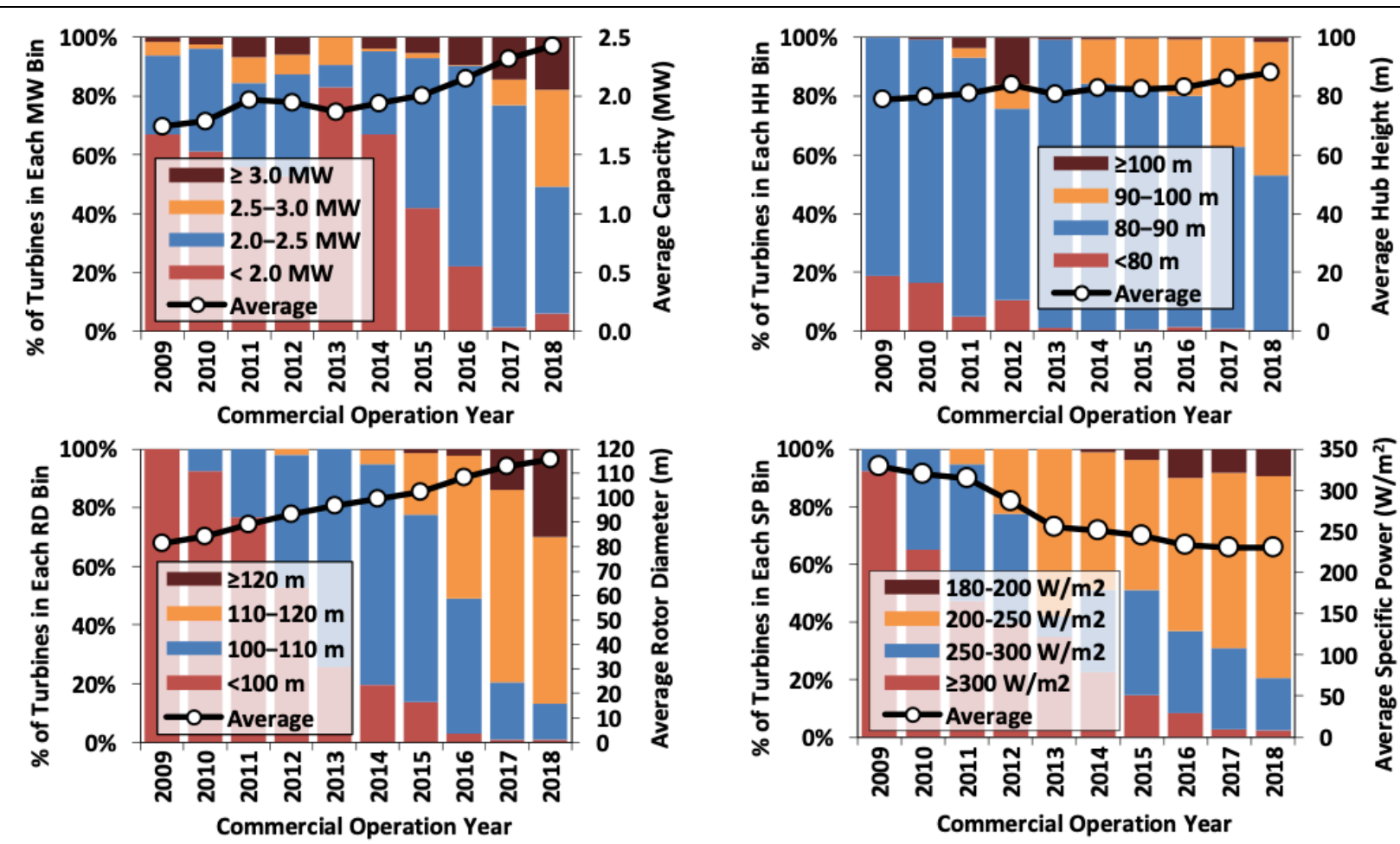

Figure 1. Trends in turbine capacity, hub height (HH), rotor diameter (RD), and specific power (SP)

Figure 2 highlights the comparative differences in the growth of these four parameters, along with the swept area of the rotor. Growing at the square of blade length, the average swept area has doubled since 2009 , greatly outpacing the $40 \%$ increase in nameplate capacity. As a result, average specific power has declined by roughly $30 \%$.

\footnotetext{
${ }^{2}$ Excerpts are denoted by an indent and text box.

${ }^{3}$ For citations in excerpt, please reference the original publication. Please note that some of the figure and table numbers are different between the original and what is reported here. This is for continuity of this document.
} 


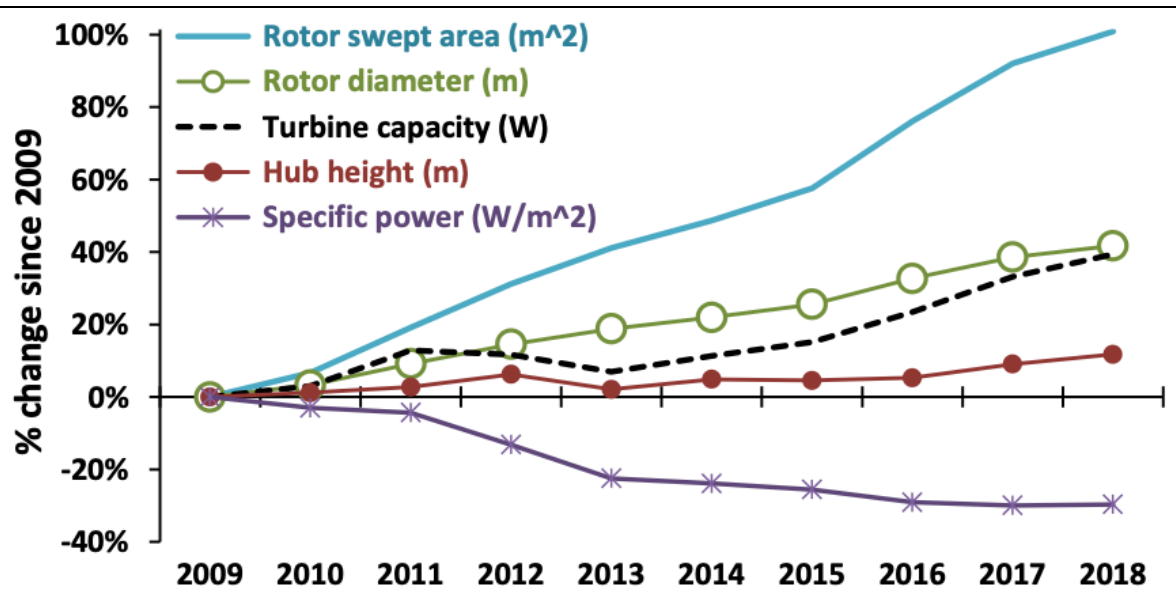

Figure 2. Cumulative percentage change in key turbine parameters since 2009

Though initially targeted at lower-wind-speed sites, low-specific-power turbines have since been deployed more broadly in the United States - in some cases even at high-wind-speed sites. For example, Figure 3 shows that by the end of 2018, projects with specific power ratings of less than $250 \mathrm{~W} / \mathrm{m}^{2}$ (yellow dots) were widespread throughout the United States, with a number of projects under 200 $\mathrm{W} / \mathrm{m}^{2}$ (red dots) operating in the high-wind-speed areas of Texas, New Mexico, Oklahoma, and Iowa (among other places). In many of these cases, a combination of relatively high site elevation (i.e., with lower air density), relatively low wind turbulence, and the capabilities of sophisticated control systems provides sufficient comfort to turbine engineers and manufacturers, as well as project developers and sponsors, that these low-specific-power turbines can withstand higher wind speeds than might have been intended by initial turbine design. 


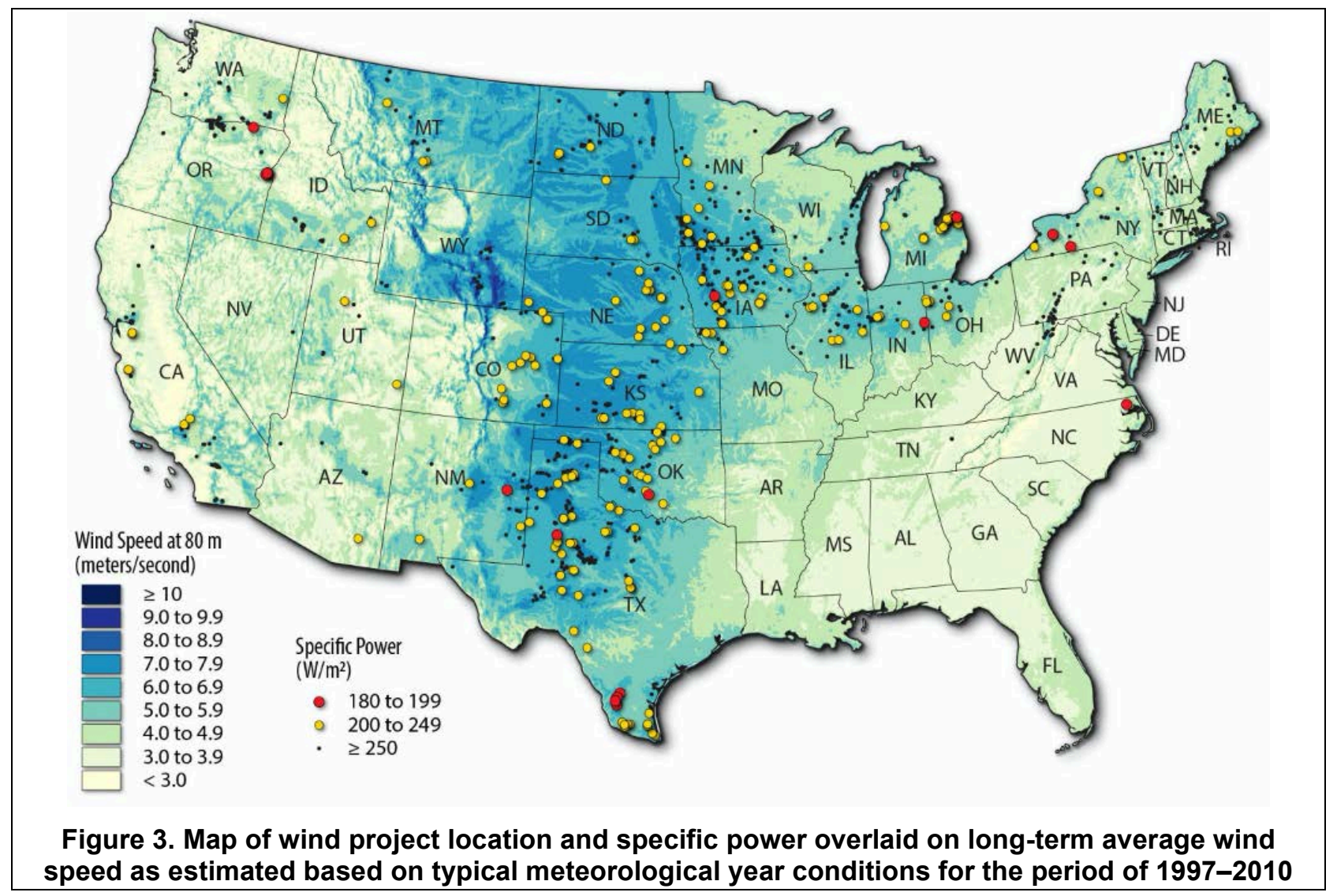

Although the United States has been a market leader in terms of deploying lowspecific-power turbines, it is not alone. China and, more recently, India and Brazil, have increasingly deployed turbines with similarly low-specific-power ratings. This stands in contrast to most European countries, where average specific power remains higher (while similarly trending downward in recent years, but from a higher level). 
Though it is difficult to generalize, the distinction between these two camps seems to be those countries with ample land area but constrained transmission (i.e., the United States, China, India, Brazil) and those with stronger grids but constrained land area (i.e., Europe). ${ }^{4}$ The "capacity-constrained" camp has an incentive to maximize the MWh generated per (constrained) MW installed-e.g., by reducing specific power-while the "land-constrained" camp has an incentive to maximize the amount of capacity installed and MWh generated per (constrained) land area, which often results in higher-capacity, higher-specificpower turbines (in light of manufacturing and transportation constraints on blade length). ${ }^{5}$

As such, in capacity-constrained markets like the United States, the trend toward lower-specific-power turbines has been driven by a desire to maximize MWh (and therefore revenue) per constrained MW through a higher capacity factor, leading to a lower LCOE. The industry's ability to achieve higher capacity factors through lower specific power is demonstrated in Figure 4, which shows projectlevel capacity factors in 2018 from 614 projects totaling 63.2 gigawatts (GW) that were installed in the United States from 2009 to 2017. Clearly, the quality of the site (as denoted by the long-term average wind speed along the $\mathrm{x}$-axis) matters for capacity factor, but so too does specific power: for any given wind speed, those projects using the lowest-specific-power turbines tend to have the highest capacity factors, and vice versa.

\footnotetext{
${ }^{4}$ Interconnection and transmission limits are not the only potential constraints on project capacity-policy can also play a role. For example, in the United States, the Public Utilities Regulatory Policy Act of 1978 requires utilities to purchase generation from "qualifying facilities," including wind projects, that are up to $80 \mathrm{MW}$ in capacity. As a result, there are many $80-\mathrm{MW}$ wind projects in the United States.

${ }^{5}$ Another potentially important differentiator between the United States and other countries with respect to specific power is the federal production tax credit (PTC) for wind power in the United States. The PTC is a 10-year income tax credit that, like any other "per-MWh" source of revenue (e.g., a power purchase agreement or feed-in tariff), financially rewards the maximization of MWh per installed MW. One key difference between the PTC and these other revenue sources, however, is that the PTC — as a tax credit rather than cash — is not readily fungible, and so must often be monetized by specialized third-party "tax equity" investors. In exchange for the project's PTCs, these tax equity investors typically provide $45-75 \%$ of the capital required to build the project, and then often drop out of the project after the 10-year PTC period has ended. This common financing structure in the United States-where tax equity investors provide the majority of up-front capital but only have an interest in the project during its first 10 years - may create an economic incentive to maximize generation, and PTCs, in the first decade of the project's life (e.g., via low-specific-power turbines), even if potentially at the expense of performance during the second decade (e.g., if there do happen to be any long-term O\&M implications from mounting ever-larger rotors on fixed generators). Though rather speculative, and also mostly outside of the scope of this paper, this notion that tax credits and financing structures might also be a driver of lower-specific-power turbines in the United States is nevertheless worth mentioning here in this footnote, if only because none of the other major wind markets-e.g., none of the European countries - support wind power through the tax code like the United States does.
} 


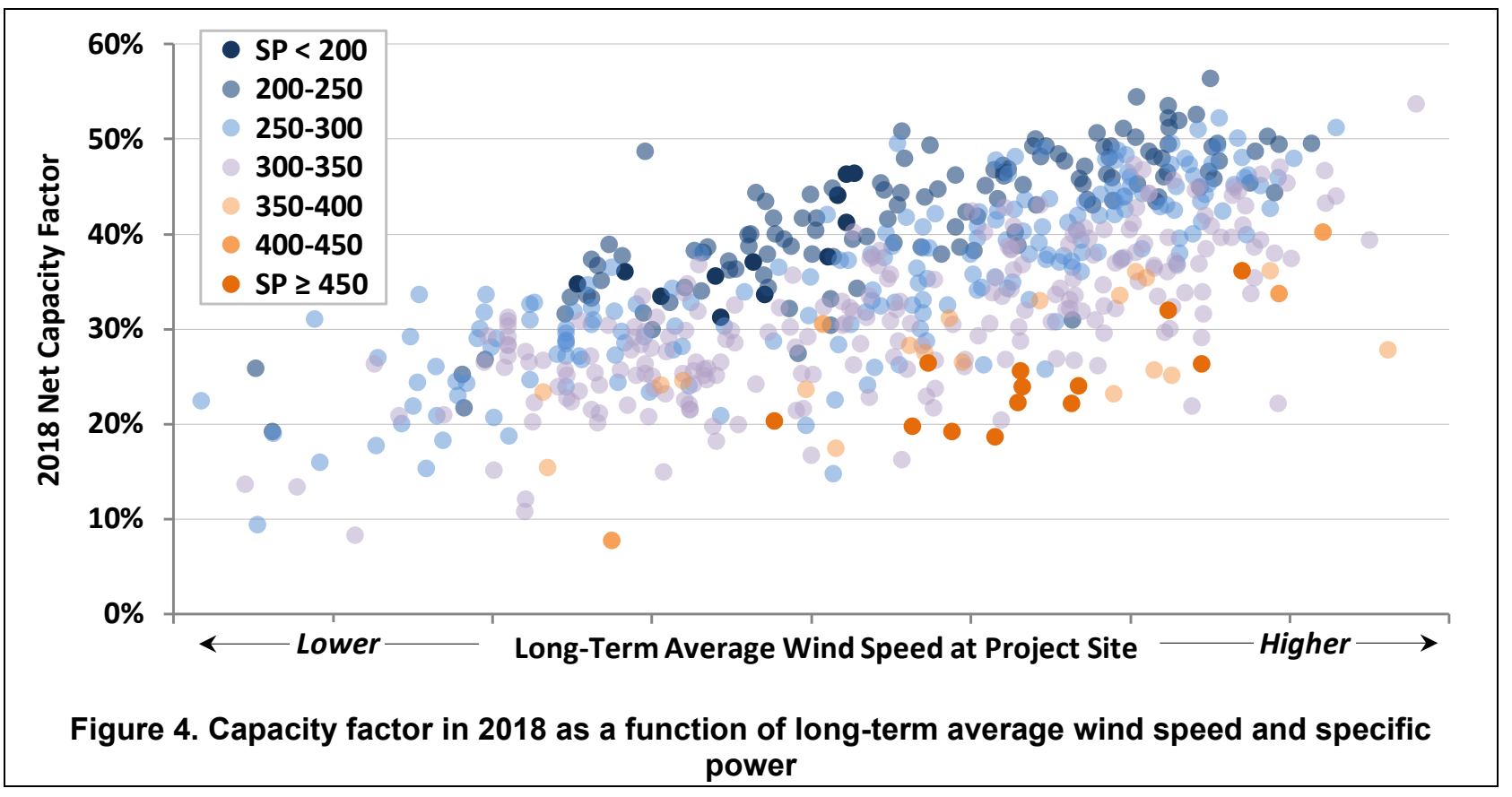

This relationship is even more evident after binning the empirical data from

Figure 5 for both wind speed and specific power, to reduce some of the inherent noise in the project-level data. In Figure 5, not surprisingly, the average capacity factor of virtually every specific power bin increases when moving from a lowerto a higher-wind-speed bin along the $\mathrm{x}$-axis. More notable, though, is that within any of the four wind-speed bins, moving from higher- to lower-specific-power turbines provides a similar or greater increase in capacity factor.

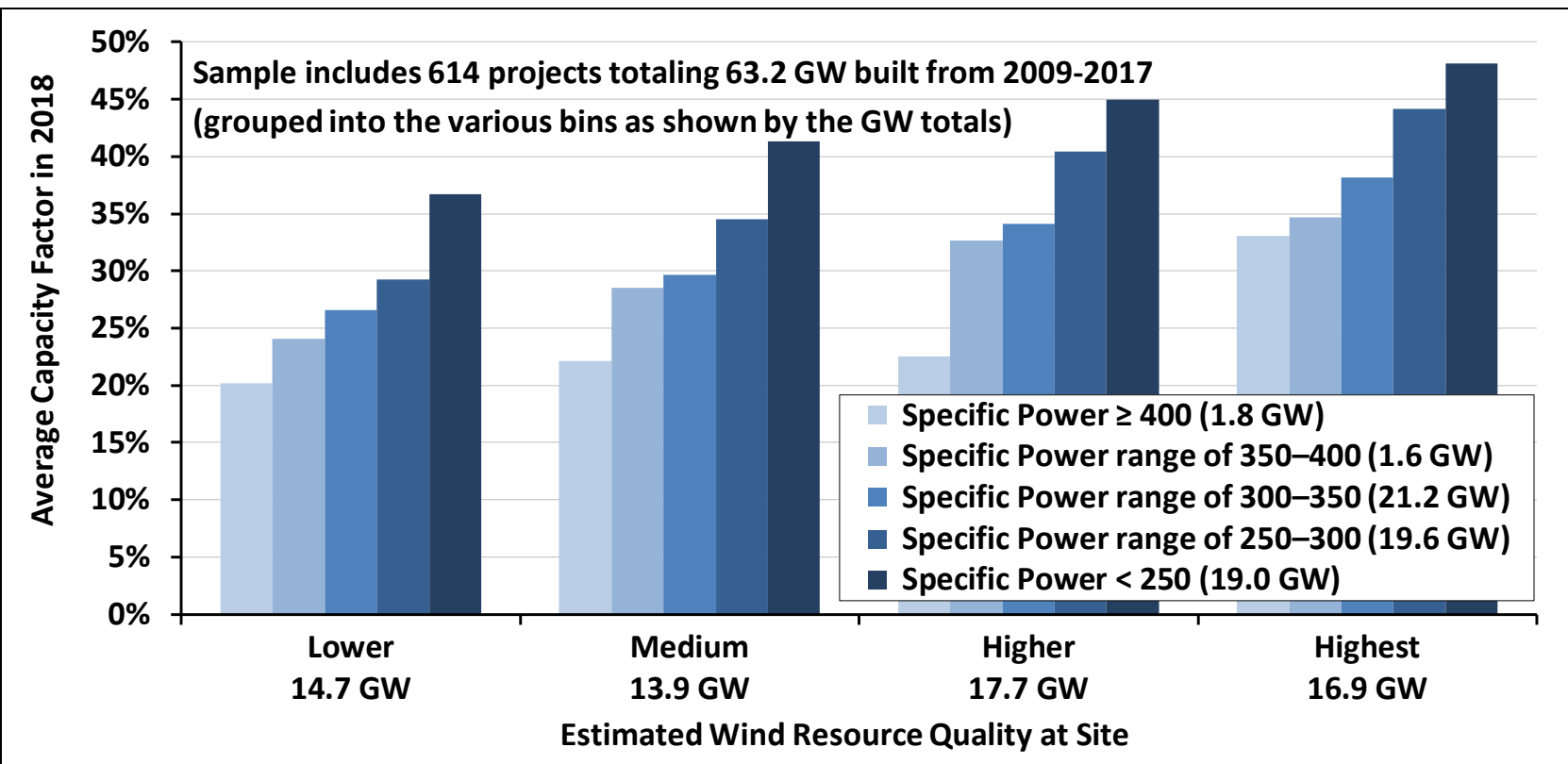

Figure 5. Capacity factor in $\mathbf{2 0 1 8}$ as a function of binned wind speed and specific power

The time trend is also instructive. Drawing upon the same wind project sample as Figures 4 and Figure 5, Figure 6 shows the average specific power of turbines 
deployed each year in the United States since 2009 (red line) — in this case, plotted on an inverse scale so that specific power moves directionally with capacity factor (the blue line). Although averages mask geospatial variations in both specific power and capacity factor over time, in general there is a strong correlation between the decline in average specific power and the increase in the average capacity factor in 2018 among more-recent project vintages.

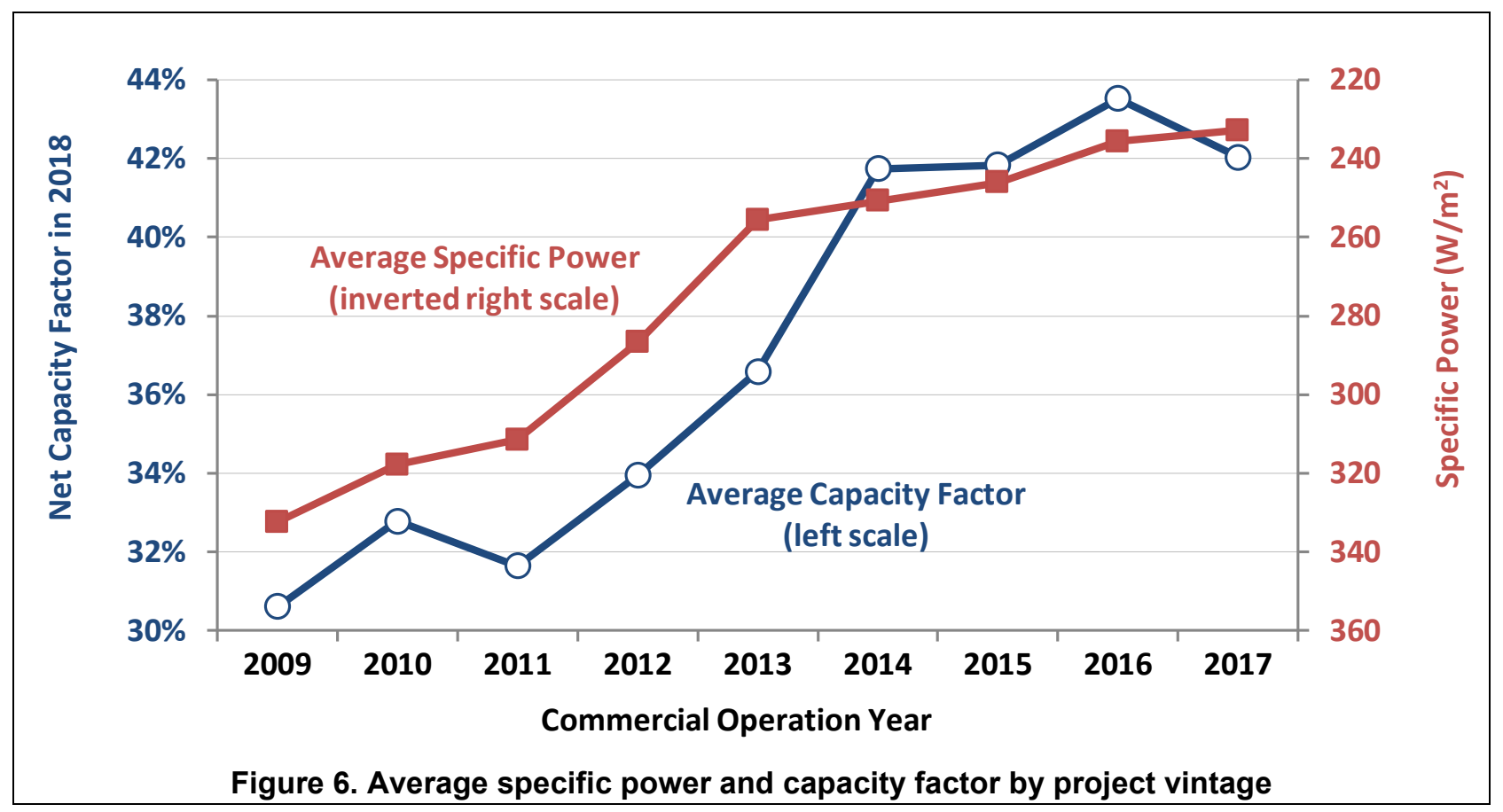

Of course, a higher capacity factor is not necessarily an end goal itself, particularly when there is a cost to achieving it - in this case, the cost of mounting a larger rotor on a given turbine. Moreover, due to the "square-cube law," the cost of a larger rotor extends beyond just the immediate first-order effects. The squarecube law states that as the diameter of a wind turbine's rotor increases, theoretical energy output increases by the square of the rotor diameter, but the volume and mass of material required to scale the rotor increases as the cube of the rotor diameter, all else being equal (Burton et al., 2001). Consequently, at some size, the cost of a larger turbine will increase faster than the resulting energy output and revenue, making further size increases uneconomical (Sieros et al. 2012).

To date, the wind industry has been able to avoid uneconomical scaling-related cost increases by streamlining manufacturing operations, optimizing turbine design, and using fewer, lighter, and stronger materials (Garrett and Rønde 2011a, 2011b; Razdan and Garrett 2018a, 2018b, 2015a, 2015b; Wiser et al. 2011).

Figure 7 employs mass data sourced from Vestas' life-cycle analyses of its 2.0 MW platform in order to plot how mass intensity (expressed three ways, from left to right: in $\mathrm{kg} / \mathrm{kW}, \mathrm{kg} / \mathrm{m}^{2}$ of swept area, and $\mathrm{kg} / \mathrm{MWh}$ ) has changed with specific 
power over time. ${ }^{6}$ Expressed in $\mathrm{kg} / \mathrm{kW}$, mass intensity has generally (and not surprisingly, given the static $2.0 \mathrm{MW}$ turbine capacity) increased with longer blades. To the extent that mass can be considered a loose proxy for cost, this increase in $\mathrm{kg} / \mathrm{kW}$ as specific power declines should push turbine costs higher on a $\$ / \mathrm{kW}$ basis. When expressed in either $\mathrm{kg} / \mathrm{m}^{2}$ or $\mathrm{kg} / \mathrm{MWh}$, however, mass intensity within each turbine class declines with specific power, enabling lower \$/MWh costs. In this way, scaling-related cost increases can still be economical, by enabling lower LCOE and power purchase agreement (PPA) prices.

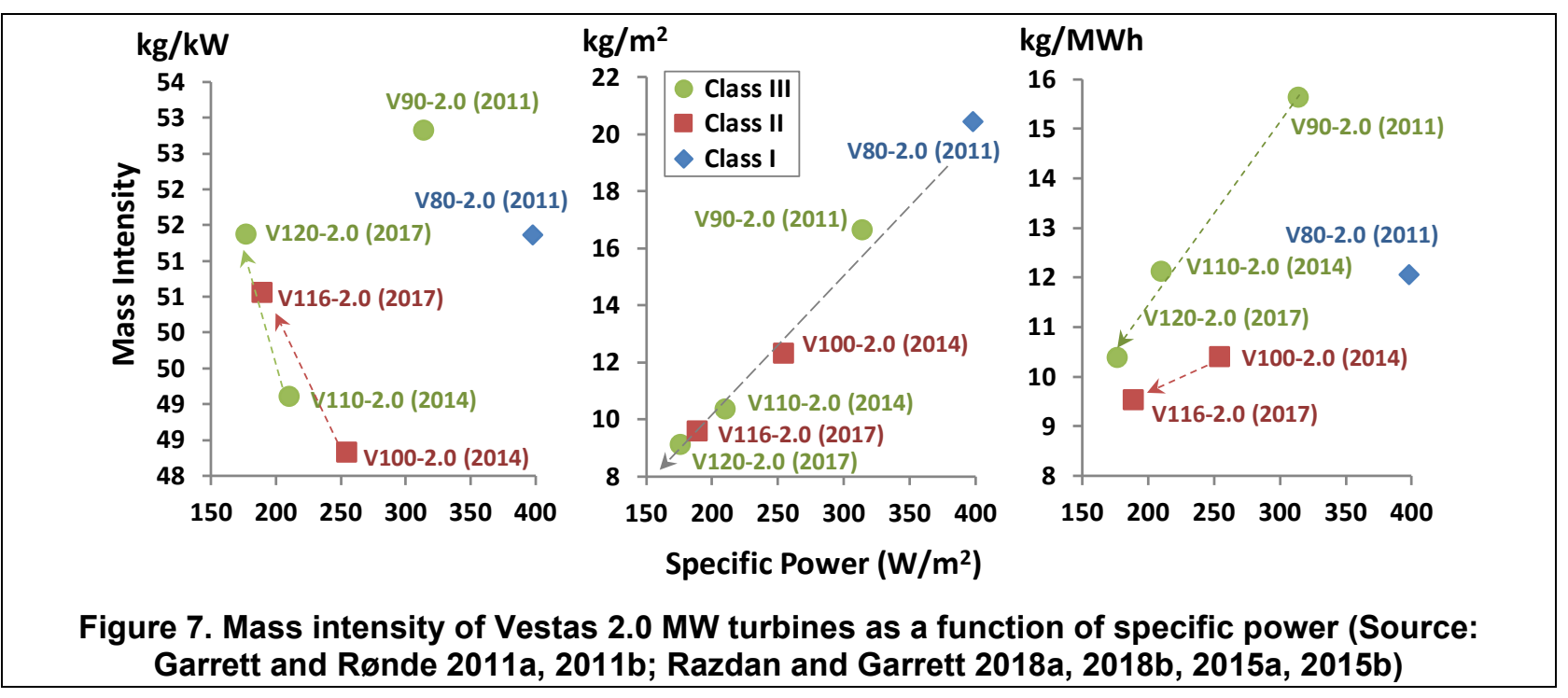

Progressing from mass intensity to cost, analysis by DOE (2015), Moné et al. (2015), Stehly et al. (2017), and Wiser et al. (2012) suggests that, at least historically, a reduction in specific power of $100-125 \mathrm{~W} / \mathrm{m}^{2}$ (i.e., between International Electrotechnical Commission [IEC] certified Class I and Class III turbines) can plausibly push turbine costs higher by $\$ 200-\$ 300 / \mathrm{kW}$. Similarly, Bloomberg New Energy Finance (2018) estimates that for a smaller specific power differential (i.e., approximately $40-50 \mathrm{~W} / \mathrm{m}^{2}$ ) between IEC certified Class II and Class III turbines, an approximately $\$ 100 / \mathrm{kW}$ cost differential could be observed in pricing data through 2018. Finally, analysis of the project-level installed cost data for projects in the United States from Wiser and Bolinger (2019) suggests a somewhat smaller premium, ranging from $\$ 90$ to $\$ 170$ for a 100 $\mathrm{W} / \mathrm{m}^{2}$ reduction in specific power.

Although the precise cost differentials between low- and high-specific-power turbines remain both uncertain (given the ranges noted in the previous paragraph)

\footnotetext{
${ }^{6}$ The mass numbers in Figure 7 include only the mass of the rotor and nacelle, and exclude the mass of the tower and foundation, solely because the life-cycle analyses from which the mass numbers are sourced (Garrett and Rønde, 2011a, 2011b; Razdan and Garrett, 2018a, 2018b, 2015a, 2015b) assume different tower heights for different turbines, resulting in an apples-to-oranges comparison. When expressing mass intensity in terms of kg/MWh, we assume hourly wind profiles with three different average wind speeds, as appropriate for each International Electrotechnical Commission turbine class - $10 \mathrm{~m} / \mathrm{s}$ for Class I, $8.5 \mathrm{~m} / \mathrm{s}$ for Class II, and $7.5 \mathrm{~m} / \mathrm{s}$ for Class III- to run through the respective power curves.
} 
and perhaps also variable (e.g., based on supply and demand for specific turbine platforms), the scaling-related cost increases experienced to date have seemingly not been enough to outweigh the LCOE benefit derived from the corresponding increase in generation. This is evident in not only the deployment trends shown earlier, but also when running the respective cost and capacity factor differentials through a simple LCOE calculator. For example, using the assumptions for operational expenditures and financing terms described later, a $100 \mathrm{~W} / \mathrm{m}^{2}$ reduction in specific power that increases capital expenditures (CapEx) from $\$ 1400 / \mathrm{kW}$ to $\$ 1600 / \mathrm{kW}$ (i.e., within the range of incremental costs from the previous paragraph) will still yield a lower LCOE with just a four-percentage point boost in capacity factor (i.e., from $36 \%$ to $40 \%$ - conservative based on the range of empirical capacity factor increases shown earlier in Figures 4-6).

By enabling a lower LCOE — even with a higher up-front cost — the trend toward lower-specific-power turbines has been one important driver of the broader trend toward lower PPA prices and LCOE in the United States over time. Figure 8 shows that these two metrics have declined on average by $60-70 \%$ since 2009 .
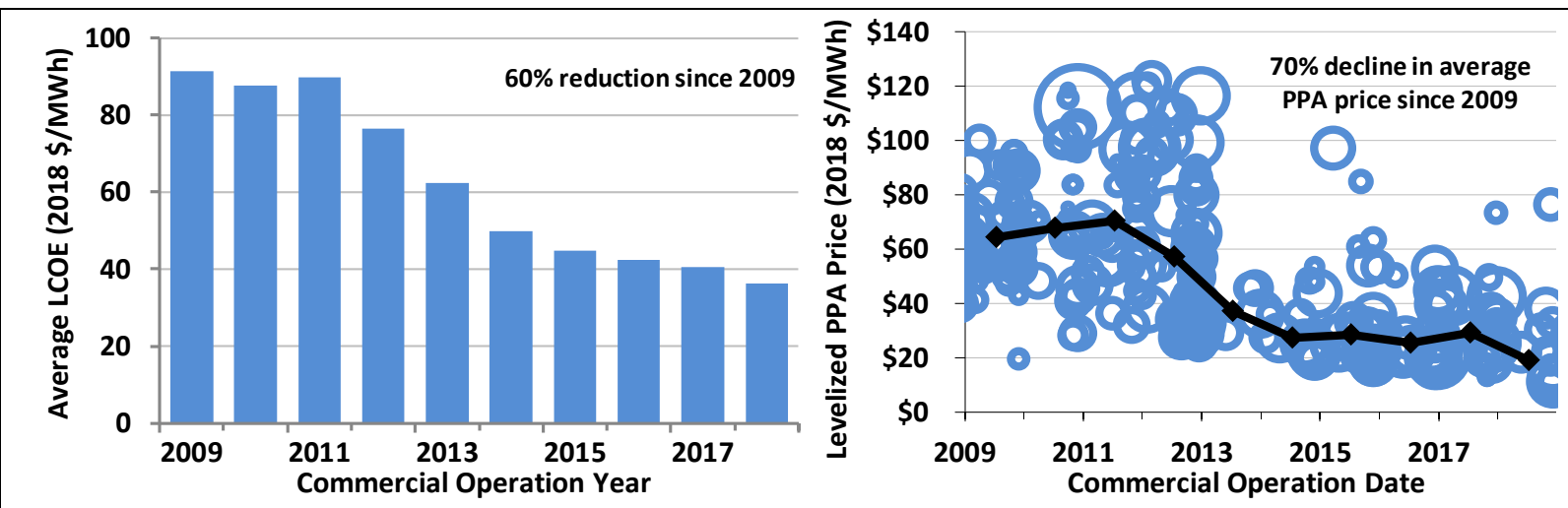

Figure 8. Trends in LCOE and levelized PPA price by project vintage (bubble size corresponds to PPA capacity)

This journal article excerpt demonstrates that wind turbines with lower specific power have provided tangible benefits to date - primarily via a higher capacity factor achieved at a cost that has enabled LCOE to decline (but also increasingly in the form of enhanced market value, which was analyzed under Task 1.B and is described next, in Section 1.2). But questions persist with respect to whether this trend will continue - or potentially even reverse - going forward. The remainder of the excerpted journal article explores these forward-looking questions through geospatial analysis; that work is described in Section 1.3 under Task 1.C.

\subsection{Subtask 1.B: Value of Low-Specific-Power Wind Turbines and High-Performance Wind Power Plants}

Work completed under Subtask 1.A demonstrates that wind turbine upscaling — namely, larger rotors and taller towers - and lower specific power have historically been the primary drivers of higher capacity factors and reduced LCOE. In turn, Subtask 1.B investigated the possibility of a "double dividend"-i.e., that taller towers and lower specific power might also enhance the 
market value of wind energy and provide other ancillary benefits. This work was ultimately published in a journal article (Wiser, Millstein, and Bolinger 2021), excerpted as follows ${ }^{7}$ :

The higher capacity factors associated with low-SP, tall-tower turbines should improve the market value of wind energy. This is because such turbines tend to shift wind generation from high wind hours - when wholesale power prices are more likely to be depressed by an oversupply of wind generation - to lower wind hours, when there is generally less wind generation and so less suppression of local wholesale power prices. The reason for this relative shift in wind production is that such turbines are specifically tuned to maximize generation in lower wind speeds (capturing more energy due to larger rotors), whereas maximum wind production is more likely to be capped (due to relatively lower nameplate capacity, relative to energy production).

We estimate the energy market value of each turbine design by pairing its hourly wind output profile with real-time hourly wholesale energy prices at the nearest pricing node. Capacity values are estimated based on the hourly wind profiles and independent system operator (ISO) -specific rules for wind's capacity credit and ISO-location-specific capacity prices or costs. We also include first-order assessments of three other benefits of low-specific-power, tall turbines: lower transmission costs, lower balancing costs, and lower financing costs.

Turning to the results of the analysis, we first compare the market value of the 2018 average turbine $\left(2.43 \mathrm{MW}, 230 \mathrm{~W} / \mathrm{m}^{2}, 88.1 \mathrm{~m}\right.$ tower) to the low-specificpower, tall-tower turbine ( $5 \mathrm{MW}, 150 \mathrm{~W} / \mathrm{m}^{2}, 140 \mathrm{~m}$ tower). Figure 9 presents our results for the percentage change in market value associated with the lowspecific-power, tall-tower design relative to the 2018 turbine, in map form and at a project level. Figure 10 presents the same basic results regionally, in histogram form, but here focusing on absolute $\$ / \mathrm{MWh}$ value changes as opposed to percentage differences.

As shown in Figure 9, the low-specific-power, tall-tower turbine tends to have higher market value than the 2018 average turbine. At the same time, there is considerable variability in this value enhancement, both across ISOs and within ISO regions. The value enhancement is as large as $+25 \%$ for some projects, while for others there is a reduction in market value by as much as $-5 \%$. The greatest value enhancement occurs in ERCOT and SPP, with moderate impacts in MISO, highly variable impacts in New England, and relatively little value enhancement for most sites in CAISO, PJM, and NYISO.

\footnotetext{
${ }^{7}$ For citations in excerpt, please reference the original publication. Please note that some of the figure and table
} numbers are different between the original and what is reported here. This is for continuity of this document. 


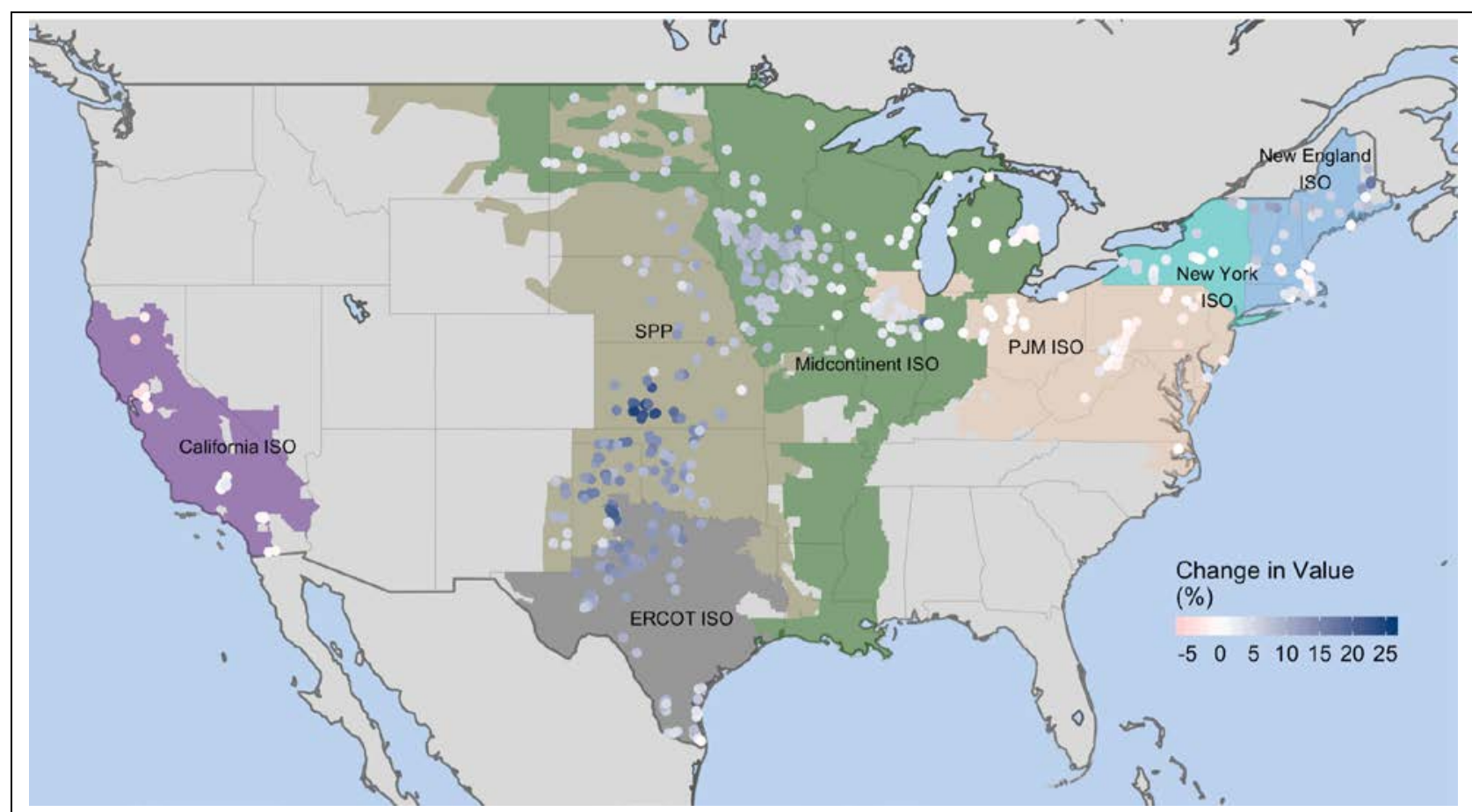

Figure 9. Percentage change in 2018 market value from low-specific-power, tall turbine vs. 2018 average turbine

The absolute value enhancement is impacted both by the percentage increase shown in Figure 9 and by average regional wholesale prices. As depicted in Figure 10, nationally, there is a normal distribution of absolute change in market value centered between a $\$ 1 / \mathrm{MWh}$ to $\$ 2 / \mathrm{MWh}$ increase in value, equivalent to a $5-10 \%$ value boost from the low-specific-power, tall turbine. ERCOT and SPP are centered on a $\$ 2 / \mathrm{MWh}$ to $\$ 3 / \mathrm{MWh}$ increase, reflecting roughly a $10-15 \%$ increase. Results from ISO-New England vary across an exceptionally large range; higher effects of as much as $\$ 6 / \mathrm{MWh}$ are found in northern New England where transmission constraints have been severe. Modest value enhancement is apparent in MISO and NYISO; results from CAISO and PJM are centered on no change in market value. 


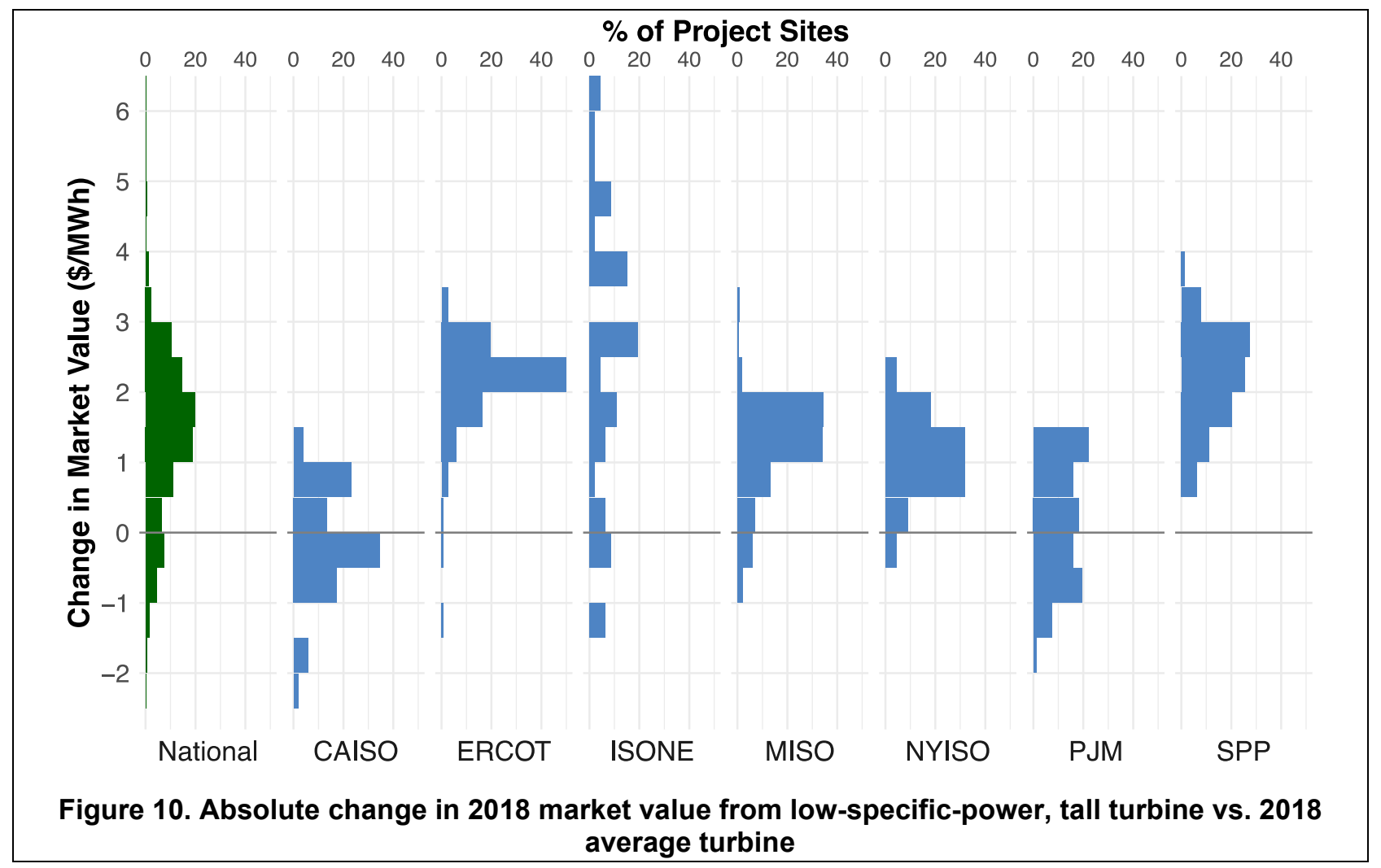

The results shown in Figure 9 and Figure 10 are impacted, to a degree, by the level of wind generation in each region. For example, wind represents $\sim 20 \%$ of total electricity supply in both SPP and ERCOT, and the value boost from lowspecific-power, tall-tower turbines is most pronounced in those two areas of the country (Figure 11).

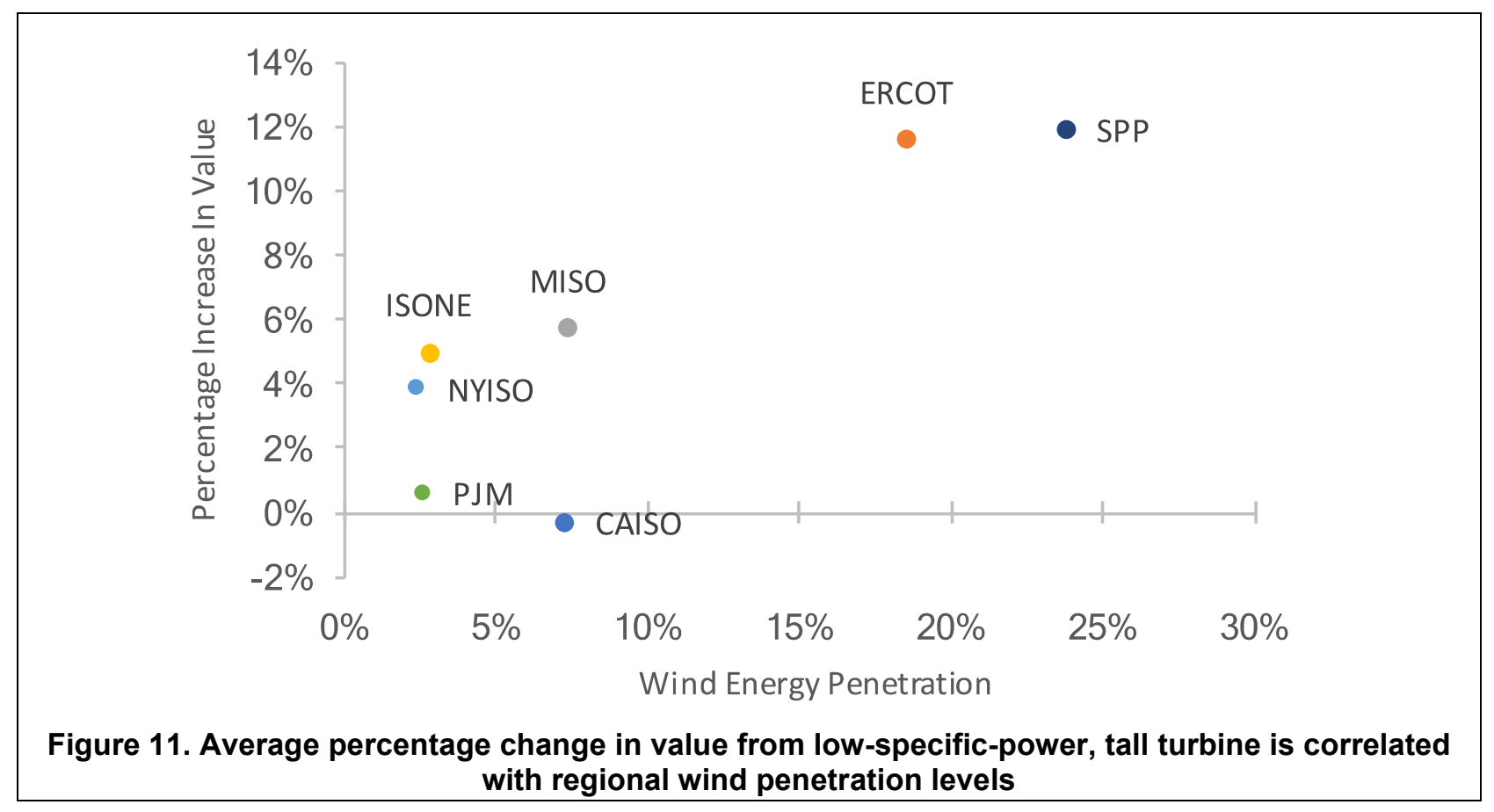


The results in Figures 9-11 are primarily driven by changes in energy value, and to a lesser extent capacity value. ERCOT, for example, runs an energy-only market, and the value enhancement shown above is therefore exclusively the result of changes in energy value. In SPP, meanwhile, we find that $77 \%$ of the value enhancement derives from the energy market, and $23 \%$ from estimated capacity value. On a national average basis, energy value represents $82 \%$ of the value boost, and capacity value $18 \%$.

Of the three additional potential benefits noted earlier-lower transmission costs, lower balancing costs, and lower financing costs - transmission savings represent the single largest opportunity (see Figure 12). Using 2018 average turbine characteristics and the assumptions described earlier, total incremental transmission costs for wind in the United States are found to equal roughly $\$ 6.7 / \mathrm{MWh}$. Applying the low-specific-power, tall-tower turbine drops these costs to $\$ 5.1 / \mathrm{MWh}$, for a savings of $\$ 1.6 / \mathrm{MWh}$. Of the $\$ 1.6 / \mathrm{MWh}$ of savings, roughly $25 \%(\$ 0.4 / \mathrm{MWh})$ accrues to the wind plant owner due to lower spur-line and interconnection costs. The remaining 75\% $(\$ 1.2 / \mathrm{MWh})$ reflects a socialized benefit from lower network expansion costs.

The potential balancing and financing advantages of low-specific-power, talltower turbines are small. For balancing, spreading the slightly greater balancing reserve requirements over much more energy with low-specific-power, tall-tower turbines leads to a benefit of about $\$ 0.2 / \mathrm{MWh}$. For financing costs, the small increase in leverage enabled by the low-specific-power, tall-tower turbine reduces the LCOE of wind by $\$ 0.30 / \mathrm{MWh}$.

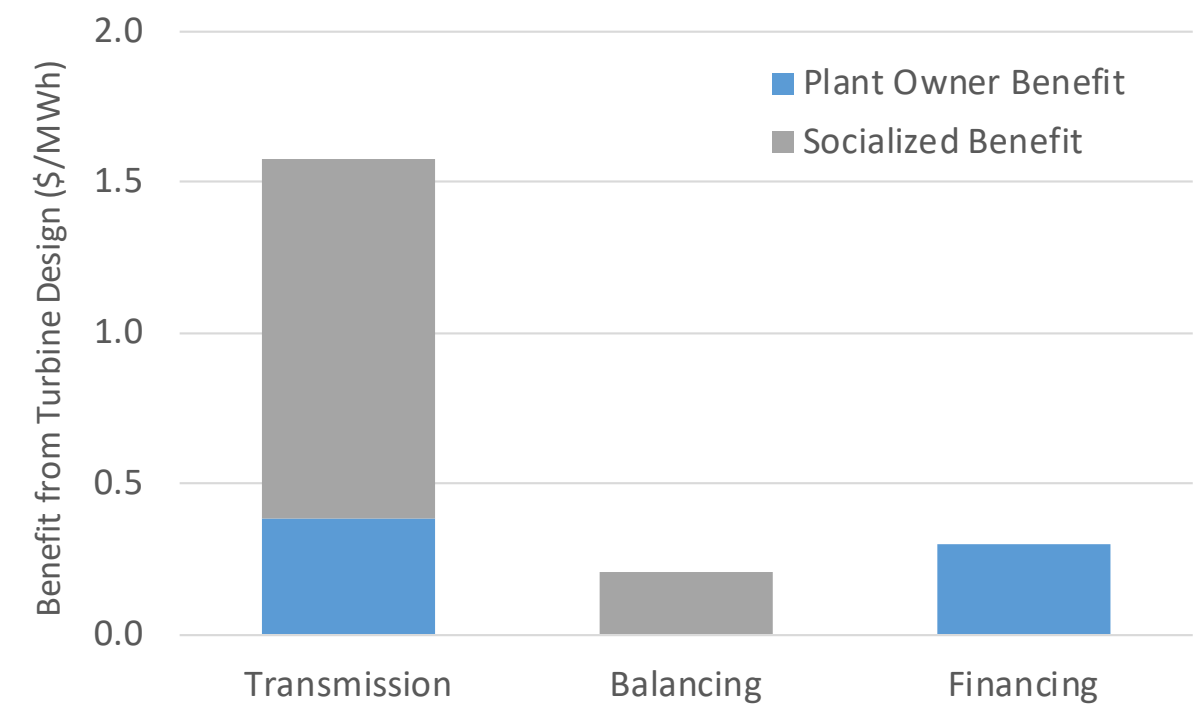

Figure 12. Transmission, balancing, and financing cost savings from low-specific-power tall wind turbines

Though wind power has expanded rapidly, continued technical advancements will be necessary if it is to reach its full potential. Thus far, the industry has focused on minimizing the direct cost of its product, the levelized cost of wind energy. In contrast, in this paper we illustrate the importance of expanding the design space 
to focus not only on direct LCOE minimization - as historically defined, and applied - but also to consider a broader set of factors, including the underlying value of wind to the electric grid. ${ }^{8}$

The scale of the potential non-LCOE benefits flowing from taller, lower-specificpower turbines are, in aggregate, relatively sizable, but also highly variable. When wind penetration reaches around $20 \%$, we find a market-value boost of $\$ 2$ $3 / \mathrm{MWh}(10-15 \%)$. This is augmented by transmission, balancing, and financing benefits, which sum to roughly $\$ 2 / \mathrm{MWh}$. For the turbines we analyzed, specific power is found to be a larger driver of these benefits than is tower height. The average LCOE of new wind projects in the U.S. in 2018 was $\$ 36 / \mathrm{MWh}$ (Wiser and Bolinger 2019). As such, this aggregate potential value enhancement of \$4$5 / \mathrm{MWh}$ from low-specific-power, tall-tower turbines is comparable to a $10-15 \%$ reduction in $\mathrm{LCOE}$.

\subsection{Subtask 1.C: National Geospatial and Market Impacts of Low- Specific-Power Turbines and High-Performance Wind Power Plants}

The purpose of Subtask 1.C was to visualize the impacts of low-specific-power wind turbine technology given a break-even analysis of this type of technology. Following is an excerpt from Bolinger et al. (2020) focusing specifically on the section titled, "Looking Ahead"?:

To quantify the change in capacity factor and LCOE associated with the turbine configurations studied, the analysis relies on hourly time series wind-speed data from NREL's WIND Toolkit ${ }^{10}$. The toolkit data can be briefly characterized as a national mesoscale wind-resource data set that includes meteorological data for more than 1.85 million locations in the contiguous United States. Each pixel in the data set reflects a $2 \times 2 \mathrm{~km}^{2}$ grid cell. The toolkit provides 7 years of time series wind-speed data derived from model simulations of the weather patterns from the historical period of 2007-2013. These model simulations use real-world historical data to create synthetic representations of the mesoscale meteorological phenomena for the period of time from which the model input data are drawn. Although there are multiple hub heights available, we focus primarily on the data for $140 \mathrm{~m}$ (based on our turbine and hub height selections described below). Calculated capacity factors reported here reflect the multiyear mean capacity factor based on all 7 years of available WIND Toolkit data and include all pixels within the contiguous United States. This particular analysis does not consider exclusions of any particular location, even though there are areas where wind

\footnotetext{
${ }^{8}$ Please note that the next paragraph from the original text was removed for clarity.

${ }^{9}$ Please note that some paragraphs are skipped here for clarity, and for citations in excerpt, please reference the original publication.

${ }^{10}$ The development of the Wind Integration National Dataset (WIND) Toolkit (https://www.nrel.gov/grid/windtoolkit.html) was funded by the Wind Energy Technologies Office within the US Department of Energy's Office of Energy Efficiency and Renewable Energy, and was created through the collaborative efforts of NREL and 3TIER (which has since been acquired by Vaisala_http://knowledge.vaisala.com/3TIER, accessed 1 September 2019).
} 
development is either unlawful (such as national parks) or impractical (such as very steep slopes and urban city centers).

To estimate annual energy generation and gross capacity factors, the WIND Toolkit's hourly wind-speed data were applied to wind turbine power curves derived from the turbine configurations detailed in Table $2^{11}$. Net capacity factors were estimated based on the application of a simple $16.7 \%$ loss adjustment. This adjustment has been used extensively in national supply curve characterizations by DOE and NREL (Cole et al., 2018; DOE, 2008, 2015; Lantz et al., 2019; Stehly et al., 2017) and is intended to reflect a combination of array and electrical losses, as well as turbine downtime.

\begin{tabular}{|c|c|c|c|c|}
\hline \multicolumn{6}{|c|}{ Table 1. Turbine Configurations Applied in National Supply Curve Modeling } \\
\hline & 2018 Average & Constant SP & Low SP & High SP \\
\hline Nameplate Capacity (MW) & 2.43 & 5.0 & 5.0 & 5.0 \\
\hline Rotor Diameter (m) & 115.6 & 165.8 & 206 & 153.5 \\
\hline Hub Height (m) & 88 and $140^{*}$ & 140 & 140 & 140 \\
\hline Specific Power (W/m²) & 231.5 & 231.5 & 150 & 270 \\
\hline
\end{tabular}

Figure 13 shows the absolute net capacity factor distributions for each of these four turbines across the United States, while Figure 14 shows the relative differences in these distributions, focusing on just the Low SP and High SP turbines relative to the Constant $S P$ turbine.

${ }^{11}$ Table 1 in this report 


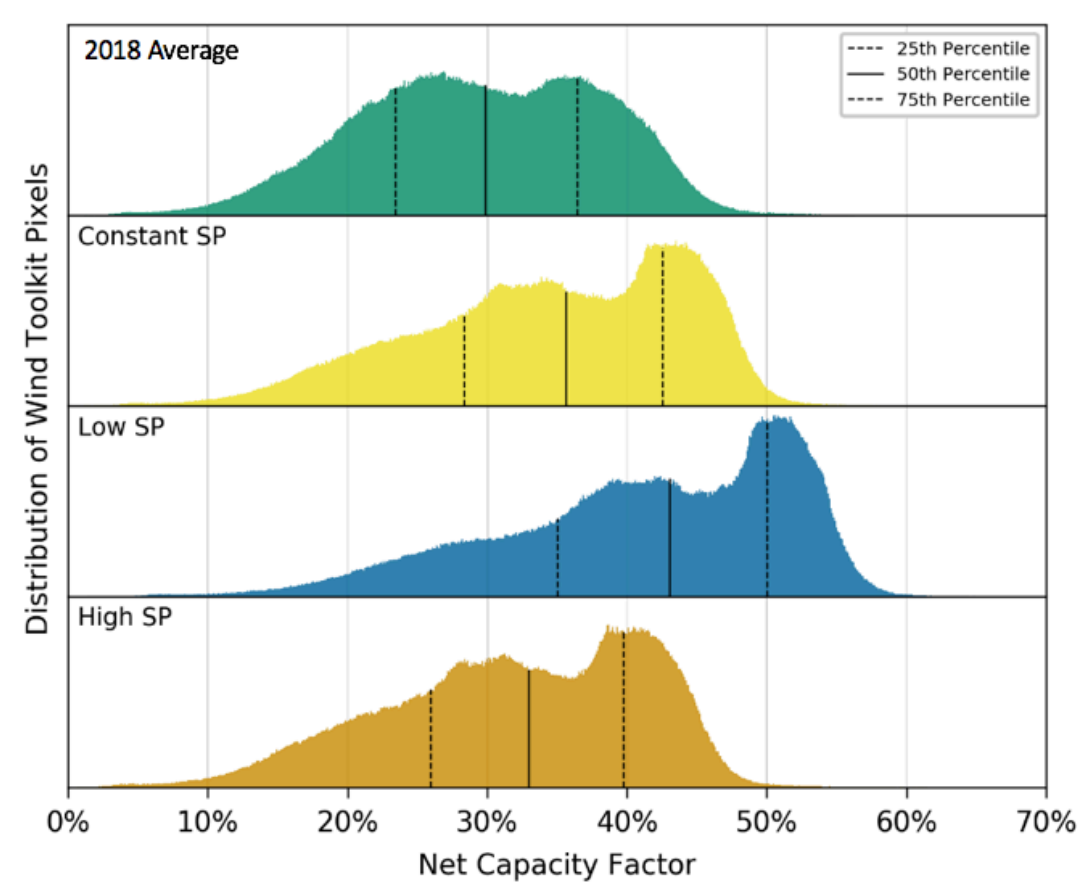

Figure 13. Net capacity factor distributions for each of the four turbines analyzed

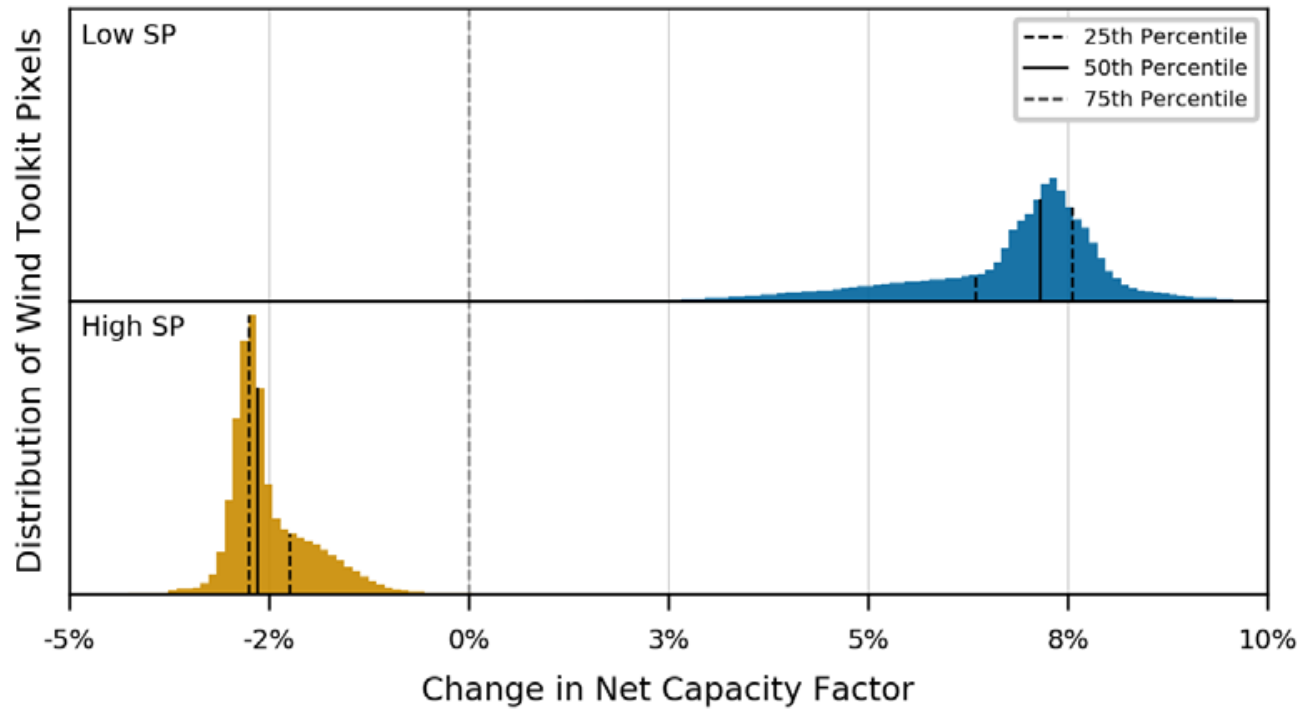

Figure 14. Net capacity factor of Low SP and High SP turbines relative to the Constant SP turbine

The Reference scenario generally aligns with traditional scaling theoryassuming equivalent site and design requirements - as well as the analytical and empirical cost estimates cited earlier (Bloomberg New Energy Finance, 2018; DOE, 2015; Mone' et al., 2015; Stehly et al., 2017; Wiser and Bolinger, 2019; Wiser et al., 2012), which hold that lower SP turbines will have higher up-front 
costs (all else being equal). Informed by these prior estimates, this scenario pegs the Low SP and High SP CapEx at 8\% above and below, respectively, the Constant SP CapEx. This results in a CapEx span of US $\$ 240 / \mathrm{kW}$ between Low SP and High SP, which falls within the range of cost premiums suggested by prior analysis of blade scaling.

The Favor Low SP scenario holds CapEx constant at US $\$ 1500 / \mathrm{kW}$ for all three turbine configurations, and therefore highlights how the differences in capacity factor shown in Figures 9 and 10 would translate to LCOE, all else being equal. Although somewhat in conflict with traditional scaling theory (i.e. the squarecube law), this scenario is considered plausible for two primary reasons. First, turbine manufacturers can potentially influence and reduce costs through supply chain optimization, including high-volume purchases. Second, higher SP turbines designed to extract maximum energy from very strong and turbulent wind conditions could potentially require greater structural strength and, hence, ultimately more materials and mass, increasing their overall cost. The Favor Low SP scenario assumes that the Low SP turbine benefits substantially from supply chain optimization and volume, and therefore costs the same as the Constant SP turbine. Conversely, it also assumes that the High SP turbine that would be commercially available is penalized by increased strength requirements and low volume, and therefore loses any inherent cost advantage it might otherwise have (in theory) relative to the Constant SP turbine.

For a final perspective on the relative economics of lower versus higher SP turbines, we turn to a metric known as the "breakeven cost," described by Lantz et al. (2019). In short, the breakeven cost equals the incremental CapEx premium or savings that a given turbine configuration (in this case, the Low SP and High SP turbines) would need to achieve at a given site in order to be competitive with a reference turbine (in this case, the Constant SP turbine) in terms of LCOE. Breakeven costs are a useful way to evaluate the magnitude of the potential challenge or opportunity associated with a given turbine configuration, without requiring a bottom-up cost estimate to compute actual competitiveness. In this case, the differences in capacity factors between the Low SP and High SP turbines relative to the Constant SP turbine (from Figure 14) are used to calculate the difference in CapEx - that is, the breakeven cost - required for the Low SP and High SP turbines to achieve an equivalent LCOE as the Constant SP turbine.

Figure 15 shows the breakeven cost results. The capacity factor improvement offered by the Low SP turbine provides a significant margin - that is, with a median exceeding US $\$ 400 / \mathrm{kW}$ of incremental CapEx - for maintaining competitiveness with the Constant SP turbine. This breakeven cost is higher than even the upper end of the range of cost premiums incurred by low-SP turbines noted earlier, providing some justification for the historical deployment of such turbines. ${ }^{12}$ Conversely, with a median breakeven cost

\footnotetext{
12 That said, due to increasingly binding manufacturing and transportation constraints associated with larger blades, dropping from 231.5 to $150 \mathrm{~W} / \mathrm{m} 2$ (as reflected in the top pane of Figure 15) is likely to be more challenging from a cost perspective than was the same $81.5 \mathrm{~W} / \mathrm{m} 2$ decline from 313 to $231.5 \mathrm{~W} / \mathrm{m} 2$ (as reflected, at least generally, in the cost premiums incurred by low SP noted earlier in this article).
} 
approaching negative US $\$ 150 / \mathrm{kW}$, the High SP turbine must - due to its lower capacity factor-demonstrate sizable cost savings per unit capacity in order to be competitive with the Constant SP turbine on an LCOE basis. Of course, depending on the degree of innovation realized and potential scaling effects, costs for low-SP technology that exceed these breakeven levels are certainly possible, as are pathways that could enable relatively lower cost per $\mathrm{kW}$ values for higher SP technology.

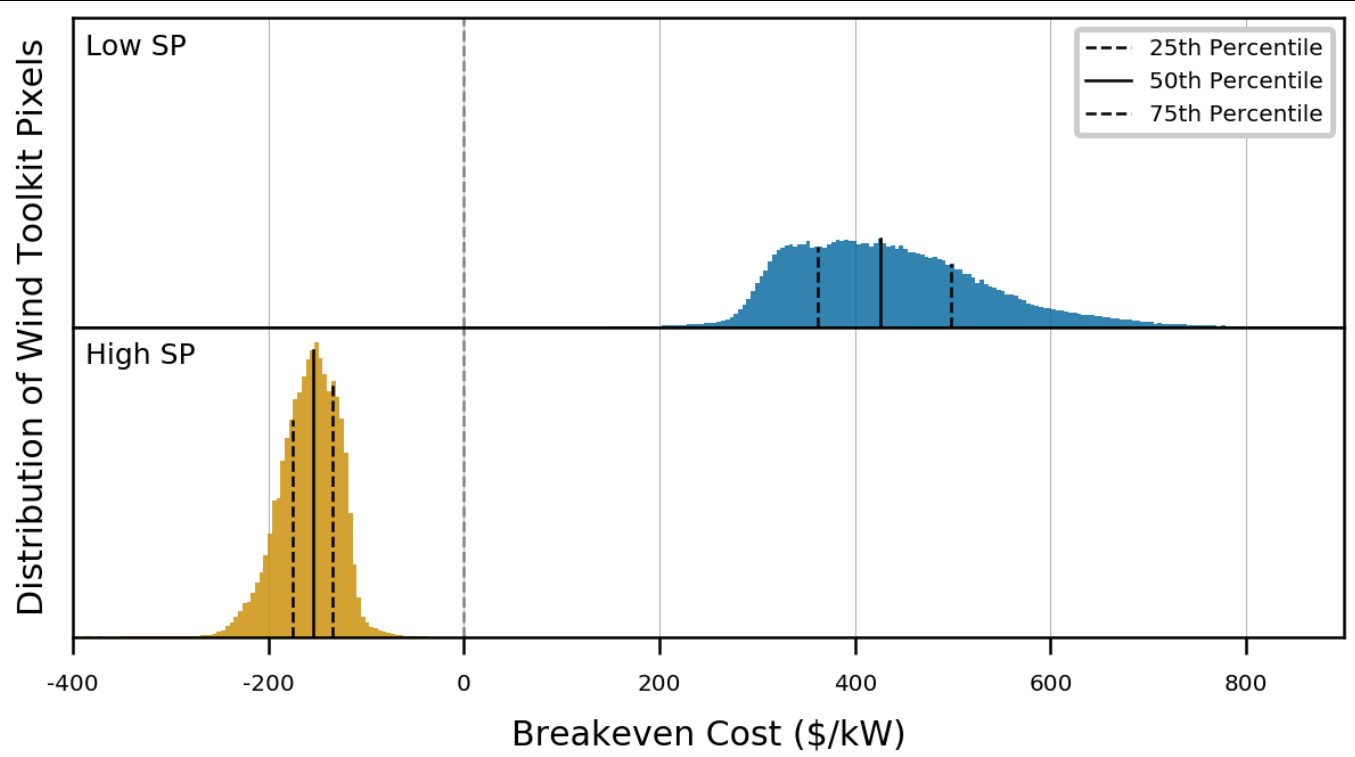

Figure 15. Relative change in CapEx value that would result in an equivalent LCOE as the Constant SP turbine

The emergence and subsequent prevalence in many markets around the world of low-SP wind turbine technology over the past decade is a notable development and indicative of the rapid pace of technological advancement within the wind industry. In relatively capacity-constrained markets, lower SP has been the most direct way to boost energy and revenue per invested dollar. In addition, lower SP has supported large reductions in LCOE as innovation in blade design and materials has enabled turbine manufacturers to minimize scaling-related cost increases associated with larger rotors, thereby enabling the higher capacity factors of low-SP technology to translate into lower LCOE. Looking ahead, however, the future of low-SP technology is not altogether clear, as new commercial offerings with relatively higher SP demonstrate that turbine design is a system optimization with competing objectives that may or may not drive toward lower SP.

In this context, the geospatial analysis conducted herein suggests that under reasonable cost scenarios, low-SP turbines could continue to play an important role in the US and global markets going forward. Even under conditions that strongly favor high SP (e.g. by penalizing low SP by US $\$ 480 / \mathrm{kW}$ ), low-SP turbines could still be preferred (on economic grounds) in many moderate to low wind-speed niches. At the same time, there are conditions under which a shift toward higher SP could be anticipated, depending on the degree of cost savings per unit capacity offered by higher nameplate capacity turbines. Nevertheless, so 
long as the relative cost premium for low-SP technology can be restrained, and costs to boost generator capacity remain at or near current levels, the future for low-SP technology and large-rotor turbines more generally could be bright, especially as existing transmission capacity is increasingly consumed by wind plants or other generators, including solar photovoltaics. Of course, a key risk associated with lower SP technology is the need to keep costs in check, particularly as turbine and blade designers must consider increasingly binding constraints in blade manufacture and transport.

Beyond these LCOE-based considerations, the boost in market value provided by low-SP turbines could also become increasingly important as wind penetration continues to grow. Further work examining this value boost from low-SP turbines at wind project sites across the United States is underway and is expected to provide greater insights into the relative economics of both large rotor turbines generally, and low-SP technology, specifically. Other future work that could provide insights into optimal SP is bottom-up techno-economic cost modeling focusing on the incremental system costs associated with rotor and generator scaling and considering the enhanced operational sophistication of modern turbine control systems. Such efforts could help to inform estimates of actual cost differences between lower and higher SP technology, the value of specific innovations that could affect SP, and the potential tradeoffs between turbines optimized for capacity- or land-constrained markets versus unconstrained turbines optimized to minimize LCOE. 


\section{Task 2: Wind Turbine Blade Cost Reduction Road Map Study}

LBNL retained DNV GL Energy USA, Inc. (DNV GL) to study the key challenges associated with manufacturing and deploying next-generation, increasingly larger, land-based wind turbine blades. Specific elements of the scope of work included:

- Hosting a workshop to solicit project input (March 6-7, 2018, Washington, D.C.)

- Issuing a public request for information (May 8, 2018)

- Developing bounding assumptions and modeling scenarios

- Developing wind turbine system models for increasing turbine/rotor sizes

- Modeling blade dimensions, costs, weight, and power performance for various sizes and design/manufacturing options

- Developing cost data and logistics break points for various transportation methods, onsite manufacturing, and hybrid scenarios

- Modeling LCOE for selected scenarios

- Developing recommendations concerning U.S. Department of Energy (DOE) R\&D funding priorities to realize supersize blades and significant LCOE impact

- Drafting the report that presents the Task 3 work and findings.

Following are excerpts from the executive summary of DNV GL's report on Supersized Wind Turbine Blade Study: R\&D Pathways for Supersized Wind Turbine Blades (Smith and Griffin 2019) ${ }^{13}$ :

Over the past decade, the U.S. wind energy industry has achieved significant improvements in energy production and cost efficiency driven in part by increased turbine, blade, and tower size. However, the industry is fast approaching a logistical cost and capability ceiling as turbine components become too large for existing U.S. infrastructure and transportation options to accommodate. Finding new solutions to logistical challenges associated with ever-larger components can enable the industry to achieve optimal wind levelized cost of energy (LCOE) options in every U.S. region.

This study includes quantitative analyses exploring the costs and benefits of three potential pathways to enable use of wind turbine blades that are too large to be transported using traditional methods on existing road and rail infrastructure. The three innovation pathways considered in this study are: innovative transportation, segmented (hybrid) blades, and on-site manufacturing. Analysis of these pathways is intended to ultimately identify unique, high-value R\&D the U.S. Department of Energy (DOE) could undertake to enable use of "supersized" blades.

Land-based transportation infrastructure, primarily road and rail networks, has been used exclusively to enable off-site factory manufacturing of all wind turbine

\footnotetext{
${ }^{13}$ For citations in excerpt, please reference the original publication. Please note that some of the figure and table numbers are different between the original and what is reported here. This is for continuity of this document.
} 
components with economically competitive transportation and delivery logistics. As wind turbine components and blades have increased in size over the past 30 years, the transportation industry has developed various innovative solutions to manipulate these oversized and overweight loads across the nation.

Today, the transportation industry and local infrastructure are handling large-scale wind turbines with the following broad dimensions:

- Rotor diameters: up to $134 \mathrm{~m}$

- Blade lengths: up to $67 \mathrm{~m}$

- Hub heights: up to or even beyond $100 \mathrm{~m}$ (consisting of multiple steel tube sections of $\sim 20 \mathrm{~m}$ long)

- Drive train system: up to 3-4 MW

Turbines of this size are primarily being deployed across the central U.S. where existing road and rail networks are more capable of conveying oversized components. Transportation of current large-scale turbines through the northeastern U.S. and Rocky Mountain regions has been increasingly difficult both technically and economically due to physical constraints associated with older infrastructure and mountainous terrain features, respectively. It is common for components in these regions to enter via a regional port to reduce overland transportation distances.

The ability of transportation solutions to deliver blades larger than $65 \mathrm{~m}$ is of growing concern. There are efforts underway to plan delivery of blades in the 70 $\mathrm{m}+$ range; however, the routing and equipment is increasingly specialized and added costs are being incurred for new or modified trailers, road modifications, increased road closures, police escort requirements, etc. Therefore, the industry is now at a logistical cost and capability ceiling in terms of feasible blade length for transport, with approximately $75 \mathrm{~m}$ as the perceived limit without more aggressive innovations.

The core approach of this study is a quantitative evaluation of the manufacture, transport, and erection of land-based wind turbines for blade lengths ranging from $65 \mathrm{~m}$ to $115 \mathrm{~m}$. Detailed system-level cost modeling was performed for the baseline (65-m blade) wind turbine; subsequent analysis for larger turbines focused on the cost to manufacture, transport, and install blades in the range of 75 $\mathrm{m}$ to $115 \mathrm{~m}$, with impact on LCOE as the primary metric.

As constraints exist to cost-effective scaling of current conventional manufacturing and transportation technologies within this size range, alternatives were identified and evaluated. These alternatives are designated as "Pathways," with three major categories identified for evaluation in this study:

1. Innovative transportation: Continued scaling-up of current manufacturing approach - monolithic blades with two scenario variants;

- Dimensional constraints of the blades as needed to facilitate long-haul transportation by truck or rail. Blade width and height are constrained due to significant barriers such as overpass and tunnel clearances with innovations 
including limited and controlled blade bending to enable increasing component lengths.

- Blade dimensions unconstrained with nonconventional transportation such as lighter-than-air (LTA) hybrid airships.

2. Hybrid solutions (segmented blades): These include segmented or modular blades, with major components within dimensional constraints that are manufactured with conventional methods and assembled on-site.

3. On-site manufacturing: Development of temporary or short-term factories in close proximity to wind turbine projects so that long-haul transportation from factory is avoided.

Any of these three pathways may be enabled by alternative manufacturing and materials technologies. Examples include additive manufacturing, thermoplastic blade skins, and low-cost carbon fibers.

Given the topic of supersized blades, transportation cost is a critical variable in quantifying any cost impacts or comparing alternative approaches for blade assembly or manufacturing that may be achieved under different pathways. In many alternative solutions, avoiding (or minimizing) transportation costs is a key objective, therefore the magnitude of transportation costs become part of the "budget" available to alternative scenarios. Transportation costs are also highly sensitive to the point of origin, destination, and selected route.

For this study, a series of assumptions and hypothetical facilities were developed to enable the analysis. These assumptions were intended to reflect both current industry characteristics and future development opportunities if supersized landbased turbines are realized. Results of the analysis are highly sensitive to the assumptions and our work is intended to be adaptable to enable analytical updates as market conditions change. Thus, the report intentionally endeavors to be transparent in all calculations. Figure 16 illustrates the hypothetical configuration of the manufacturing facilities and project locations used in this study. 


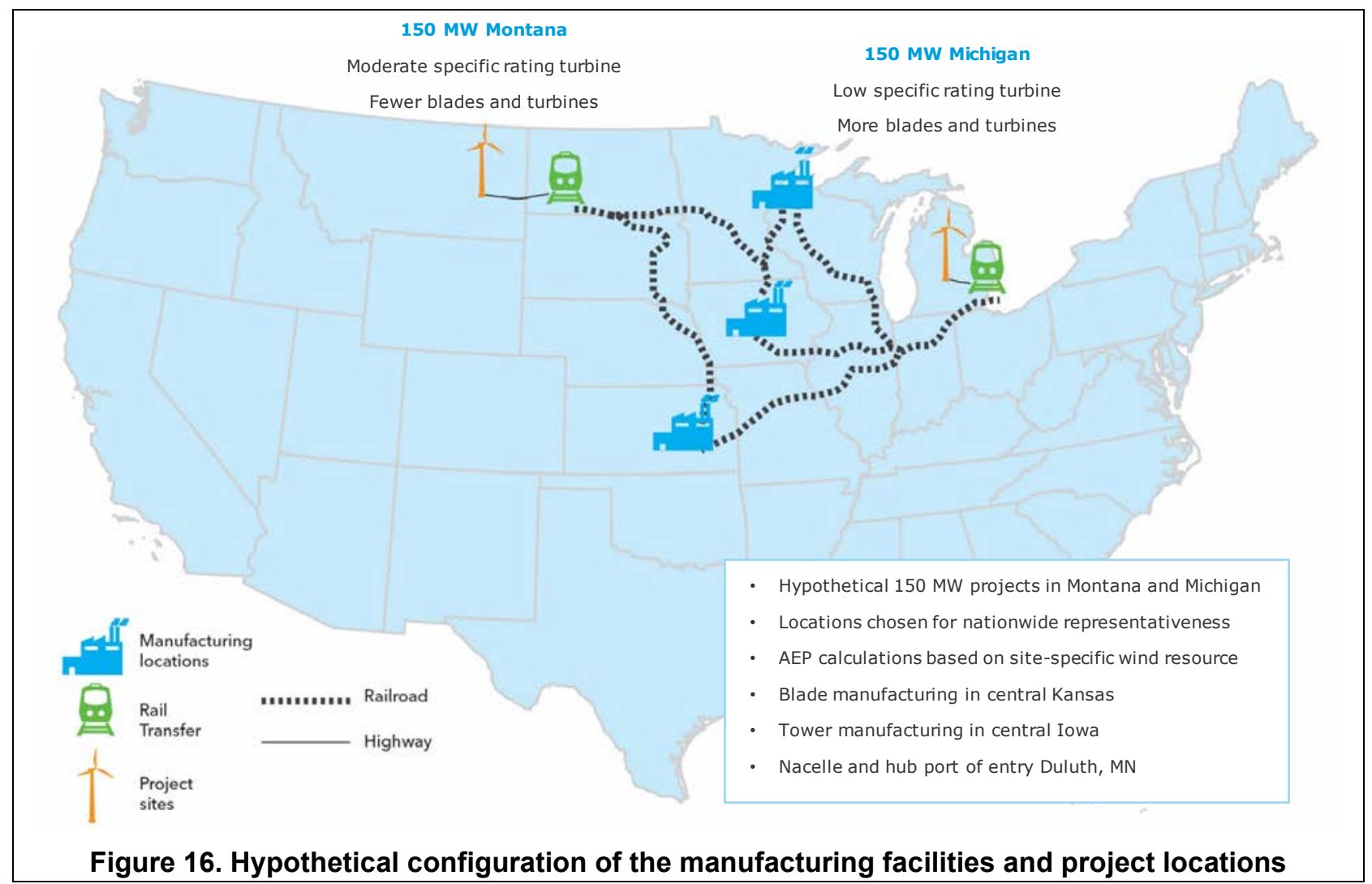

DNV GL identified and modeled two classes of wind turbines to represent current and possible near-future industry practice for optimizing turbine design to site conditions. These include a "Low Wind Speed" turbine (LWST) and a "Moderate Wind Speed" turbine (MWST) with specific power of approximately $150 \mathrm{~W} / \mathrm{m} 2$ and $225 \mathrm{~W} / \mathrm{m} 2$, respectively. These characteristics are roughly aligned with current and potential near-future competitive turbines in the U.S. market and correspond to International Electrotechnical Commission (IEC) design classification of III and II, respectively. The primary turbine blade, rotor, tower, and site wind speed parameters are given in Table 2. LWSTs (specific power of $\sim 150 \mathrm{~W} / \mathrm{m} 2$ ) are assumed to be used at the hypothetical Michigan site, and MWSTs (specific power of $\sim 225 \mathrm{~W} / \mathrm{m} 2$ ) are assumed to be used at the hypothetical Montana site. For a given rotor size, the blade designs between the LWST and MWST are unchanged; only the generator rating is modified to achieve the specific power target.

While this approach was selected as a simplification for the purposes of this study, DNV GL considers it to also be a reasonably good approximation of industry trends. Blades intended for higher-wind sites will typically have structural design dominated by peak load cases, whereas lower specific-power turbines designed for lower wind-speed sites may be more influenced by fatigue loading due to the use of relatively longer blades. 


\begin{tabular}{|c|c|c|c|c|c|c|c|}
\hline Turbine ID & $\begin{array}{l}\text { Blade } \\
\text { Length } \\
\text { (m) }\end{array}$ & $\begin{array}{l}\text { Turbine } \\
\text { quantity }\end{array}$ & $\begin{array}{c}\text { Rotor } \\
\text { Diameter } \\
\text { (m) }\end{array}$ & $\begin{array}{l}\text { Tower } \\
\text { Height (m) }\end{array}$ & $\begin{array}{c}\text { Specific } \\
\text { Rating } \\
\left(\mathrm{W} / \mathrm{m}^{2}\right)\end{array}$ & $\begin{array}{l}\text { Generator } \\
\text { Size: (MW) }\end{array}$ & $\begin{array}{c}\text { Average } \\
\text { Wind Speed } \\
\text { at Hub }(\mathrm{m} / \mathrm{s})\end{array}$ \\
\hline Baseline & 65 & $\begin{array}{l}71 \text { (MI) } \\
46 \text { (MT) }\end{array}$ & 134 & 100 & $\begin{array}{l}150 \text { (MI) } \\
225 \text { (MT) }\end{array}$ & $\begin{array}{l}2.10 \text { (MI) } \\
3.25 \text { (MT) }\end{array}$ & $\begin{array}{l}7.21 \text { (MI) } \\
7.95(\mathrm{MT})\end{array}$ \\
\hline WTG-75 & 75 & $\begin{array}{l}54 \text { (MI) } \\
35 \text { (MT) }\end{array}$ & 154 & 110 & $\begin{array}{l}150 \text { (MI) } \\
225 \text { (MT) }\end{array}$ & $\begin{array}{l}2.75 \text { (MI) } \\
4.25 \text { (MT) }\end{array}$ & $\begin{array}{l}7.38 \text { (MI) } \\
8.12 \text { (MT) }\end{array}$ \\
\hline WTG-95 & 95 & $\begin{array}{l}33 \text { (MI) } \\
22 \text { (MT) }\end{array}$ & 194 & 130 & $\begin{array}{l}150 \text { (MI) } \\
225 \text { (MT) }\end{array}$ & $\begin{array}{l}4.50 \text { (MI) } \\
6.75 \text { (MT) }\end{array}$ & $\begin{array}{l}7.69 \text { (MI) } \\
8.42 \text { (MT) }\end{array}$ \\
\hline WTG-115 & 115 & $\begin{array}{l}23 \text { (MI) } \\
15 \text { (MT) }\end{array}$ & 234 & 150 & $\begin{array}{l}150 \text { (MI) } \\
225 \text { (MT) }\end{array}$ & $\begin{array}{l}6.50 \text { (MI) } \\
9.75 \text { (MT) }\end{array}$ & $\begin{array}{l}7.92 \text { (MI) } \\
8.65 \text { (MT) }\end{array}$ \\
\hline
\end{tabular}

DNV GL performed detailed blade structural and cost modeling to derive bladespecific estimates for use in this study. Table 3 provides a summary of the estimated weight and cost for both monolithic and modular (segmented) blades produced in a traditional off-site factory. The weight and cost adders for modular blade components range from about $11.5 \%$ to $14.5 \%$ of the monolithic $75 \mathrm{~m}$ and $115 \mathrm{~m}$ blades, respectively.

\begin{tabular}{|c|c|c|c|c|c|c|c|c|c|c|}
\hline \multirow{2}{*}{$\begin{array}{c}\text { Blade } \\
\text { Length }(m)\end{array}$} & \multirow{2}{*}{$\begin{array}{l}\text { Radius } \\
(\mathrm{m})\end{array}$} & \multicolumn{2}{|c|}{ Monolithic } & \multicolumn{2}{|c|}{ Spanwise Joint } & \multicolumn{2}{|c|}{ Root Cuff } & \multicolumn{3}{|c|}{ Segmented Blade } \\
\hline & & Mass (kg) & Cost & Mass (kg) & Cost & $\begin{array}{l}\text { Mass } \\
(\mathrm{kg})\end{array}$ & Cost & $\begin{array}{l}\text { Mass } \\
\text { (kg) }\end{array}$ & & Cost \\
\hline 65 & 67 & 18,640 & $\$ 194,788$ & N/A & N/A & N/A & N/A & N/A & & N/A \\
\hline 75 & 77 & 25,314 & $\$ 264,531$ & 2,905 & $\$ 30,252$ & N/A & N/A & 28,219 & & 94,783 \\
\hline 95 & 97 & 42,071 & $\$ 439,638$ & 4,828 & $\$ 50,277$ & 370 & $\$ 4,479$ & 47,268 & & 4,394 \\
\hline 115 & 117 & 63,546 & $\$ 664,059$ & 7,292 & $\$ 75,942$ & 1,857 & $\$ 20,827$ & 72,695 & & 50,828 \\
\hline \multicolumn{11}{|c|}{$\begin{array}{l}\text { Blade-specific LCOE was then calculated for the Baseline turbine using the Total } \\
\text { Cost of Delivered Blade, Turbine Annual Energy Production (AEP), blade } \\
\text { specific operations and maintenance (O\&M) cost, and fixed charge rate (7.9\%). } \\
\text { Then, holding the Baseline blade-specific LCOE as a constant and adjusting for } \\
\text { scaled manufactured blade costs and AEP for each of the study turbines, a "Total } \\
\text { Cost of Delivered Blade Target" was derived for the } 75 \mathrm{~m}, 95 \mathrm{~m} \text {, and } 115 \mathrm{~m} \\
\text { blades. In calculating this Target, impacts of taller hub heights, larger swept area, } \\
\text { and increased wind speeds associated with larger wind turbines are accounted for } \\
\text { in the AEP for each turbine. } \\
\text { The Target represents the threshold or "budget" for pathways to compete. } \\
\text { Pathways that result in Total Delivered Blade Costs that are more or less } \\
\text { expensive than the Target value will influence the blade's contribution to overall } \\
\text { system LCOE accordingly. Pathways equal to the Target have no impact on }\end{array}$} \\
\hline
\end{tabular}


system level LCOE but may still enable LCOE improvements in other turbine systems.

Figure 17 presents the calculated Total Cost of Delivered Blade Targets for each of the project locations. The cost target in Michigan is greater than in Montana due to older infrastructure, increased number of local jurisdictions to travel through, and other transportation costs in the Great Lakes region being slightly more expensive than similar costs in Montana.

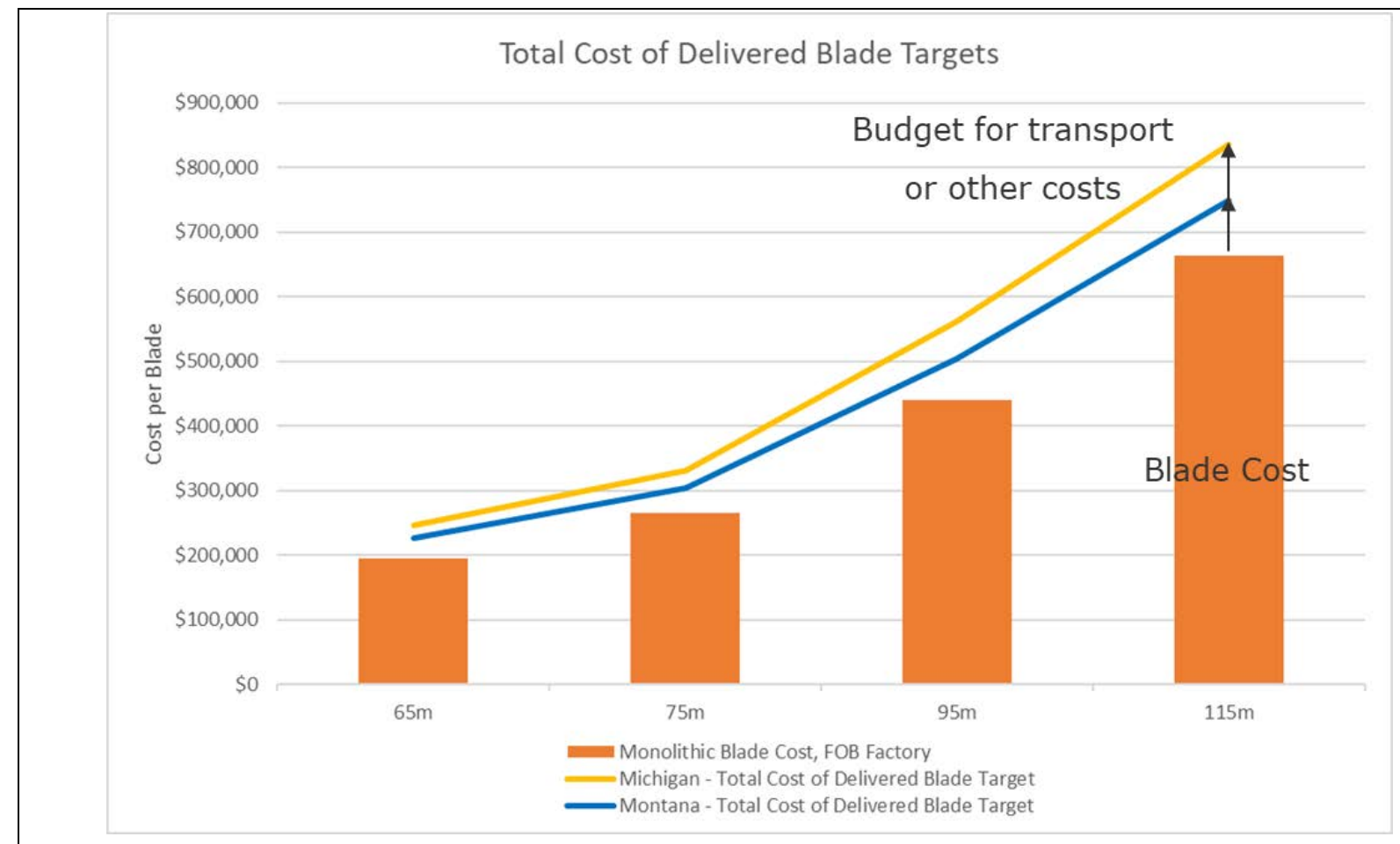

Figure 17. Calculated total cost of delivered blade targets

Figure 18 and Figure 19 summarize our estimates of the total costs of delivered blades by innovation pathway at the hypothetical projects in Michigan and Montana, respectively. The target values indicated on these figures correspond to the point at which total delivered blade costs have no impact on increasing or decreasing the system LCOE. Values below the target indicate an opportunity to help lower system LCOE, whereas values exceeding the target indicate upward pressure on system LCOE.

Figure 20 and Figure 21 summarize the percent impact on system LCOE for each of the innovation pathways at the hypothetical projects in Michigan and Montana, respectively. The impact on system LCOE accounts for total costs of delivered blades, plus includes AEP performance variations, O\&M cost differences, and fixed charge rate effects. Whether increases in system LCOE caused by supersized blades are acceptable depends on opportunities to achieve cost savings in other turbine sub-systems. Similarly, achieving a neutral impact on system LCOE can be acceptable, provided it does not come at a cost of increases in other 
parts of the turbine. Thus, integrating these results into a more holistic study of supersized wind turbines is recommended. 


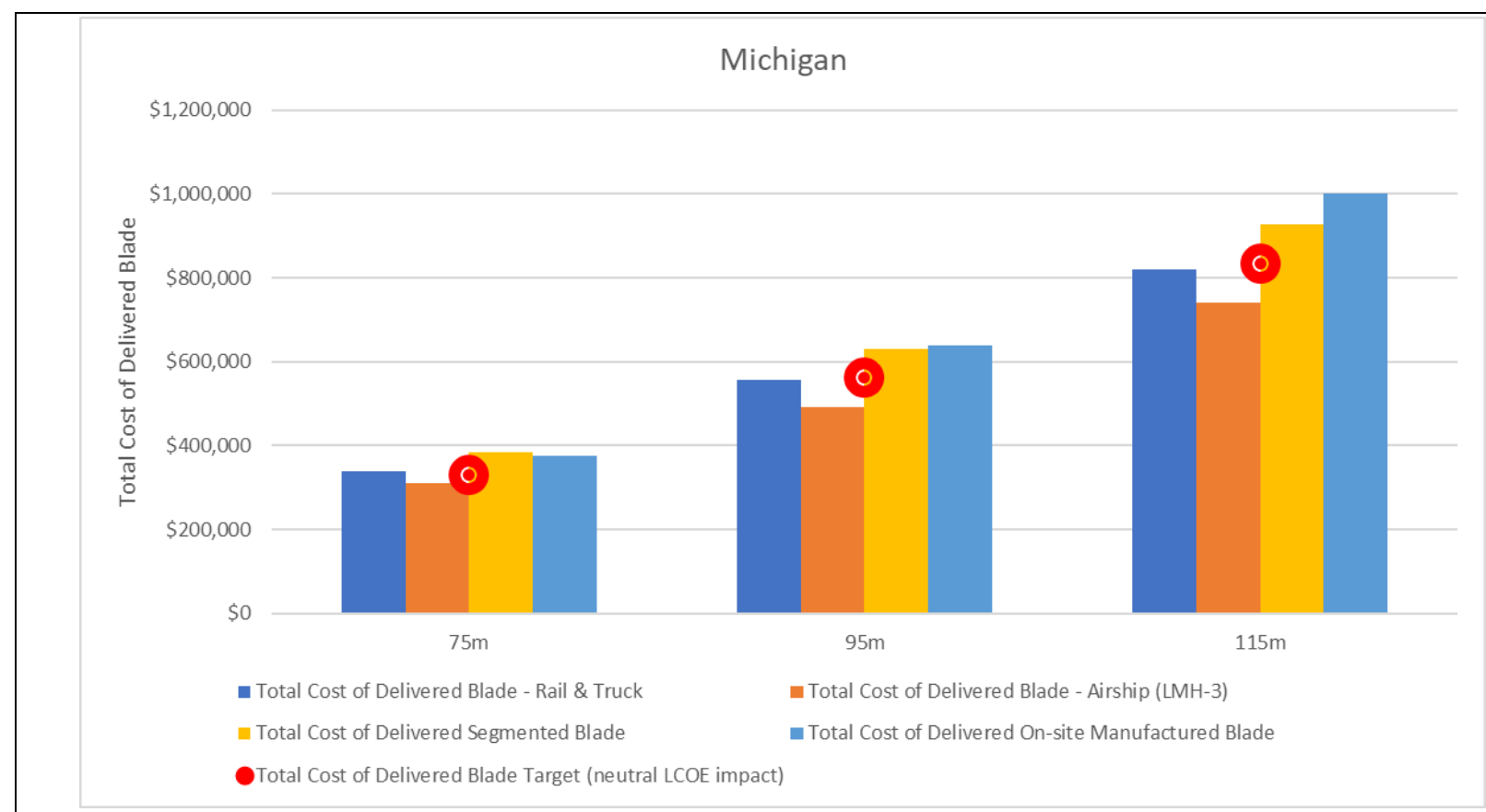

Figure 18. Innovation Pathway summary - Total cost of delivered blades, Michigan

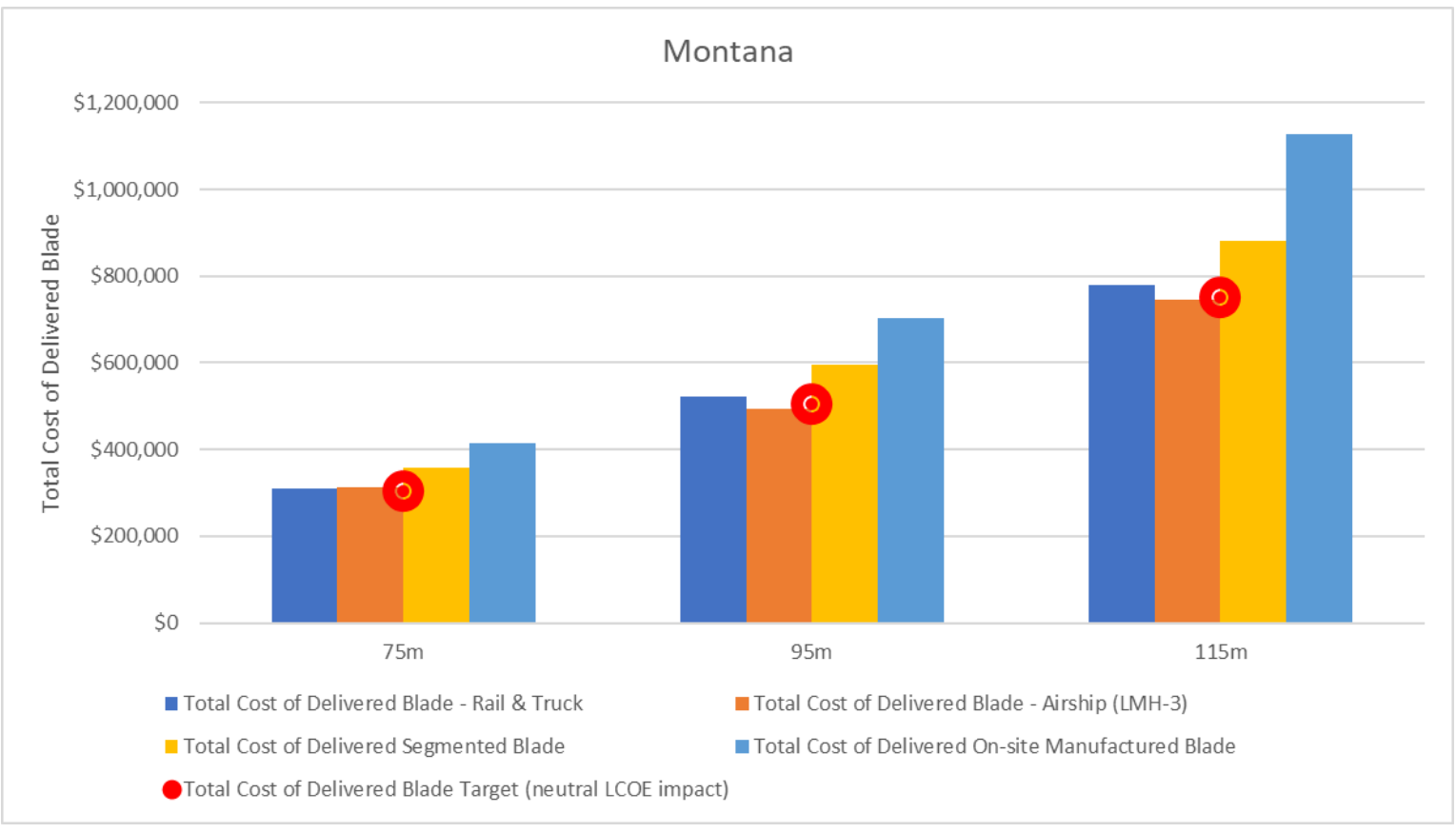

Figure 19. Innovation Pathway summary - Total cost of delivered blades, Montana 


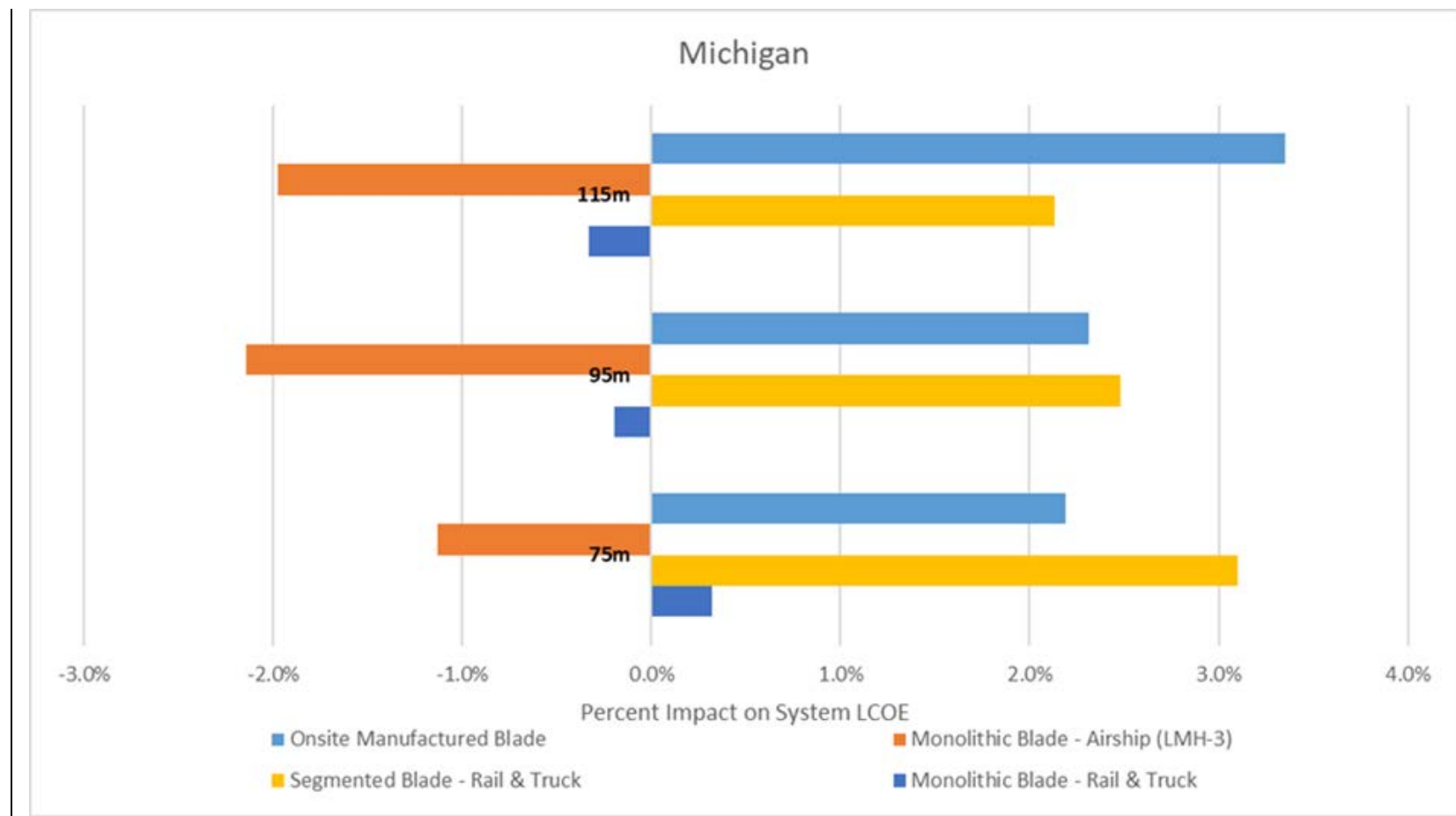

Figure 20. Innovation Pathway summary - Impact on system LCOE, Michigan

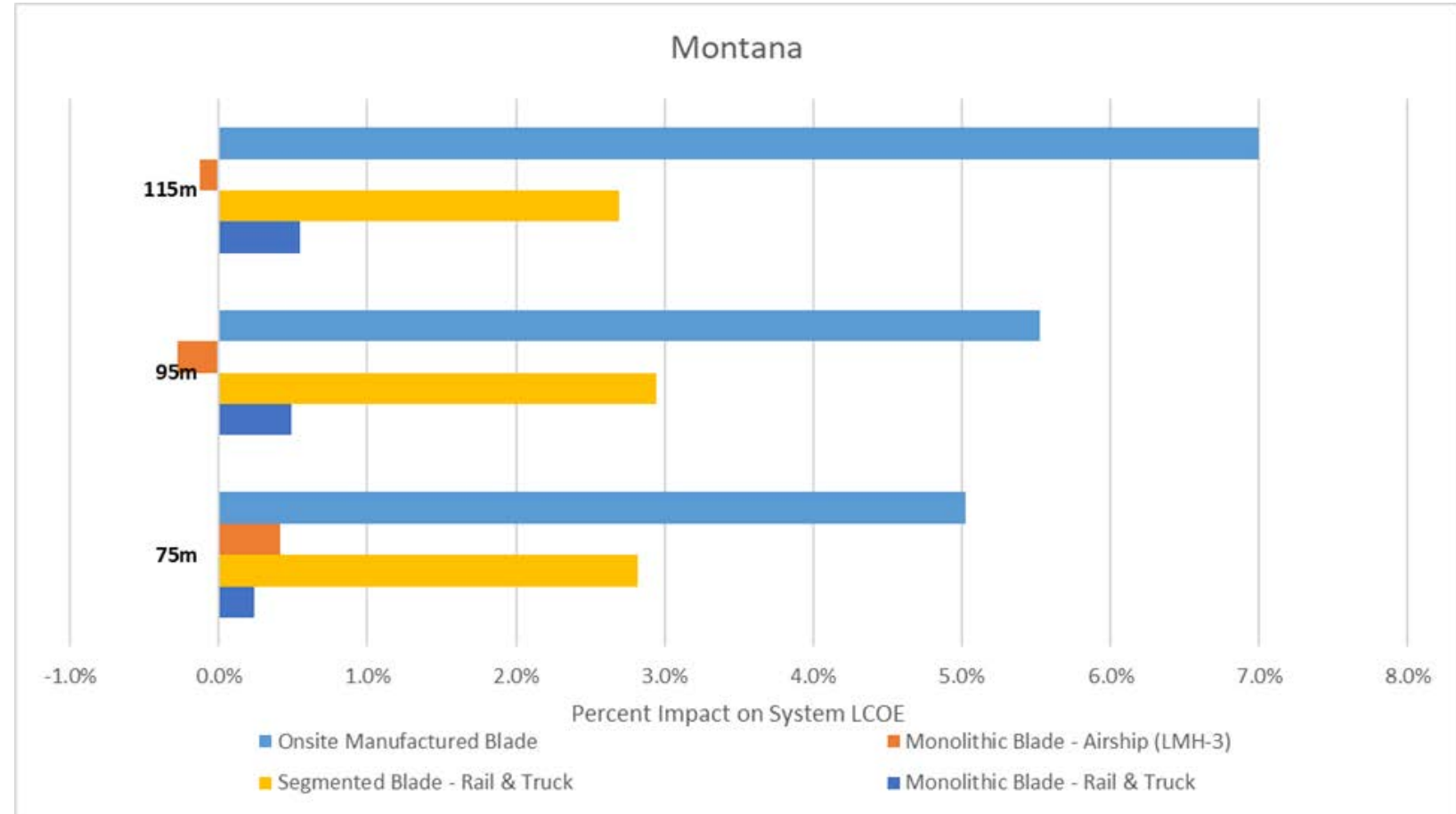

Figure 21. Innovation Pathway summary - Impact on system LCOE, Montana

Based on our analysis and the values summarized in Figure 18 through Figure 21, opportunities for supersized blades where blade-specific costs and performance contributed to a neutral or reduced overall system LCOE appear possible with use of LTA hybrid airships or controlled blade bending in rail transport. In other innovation pathways studied, blade-specific costs and performance contributed to 
increases in system LCOE indicating a broader, more systemic approach beyond just blades or different project site assumptions may be necessary to achieve lower system level LCOE.

LTA hybrid airships, under our assumptions, were identified as having potential for blade-specific costs and performance able to lower system LCOE at both project sites, ranging from $-2.1 \%$ to $0.4 \%$ depending on the site and blade length. LTA hybrid airships, if certified and commercialized, have potential to enable nationwide deployment of supersized blades and wind turbines in a cost competitive manner. Although there are active commercial efforts to bring this technology to market, there is significant uncertainty in the timing and cost of this innovation option. Monitoring developments of this technology and seeking areas of collaboration to improve market development for wind energy (and other applications) is recommended due to the potential for its enabling effects for supersized turbines.

Currently, blade flexing during transport is not allowed because this loading method has not been established in the blade design and transport infrastructure has not been developed to enable it. R\&D that enables limited and controlled blade bending in rail transport plus specialized road trailers appears able to achieve a neutral impact on system LCOE, ranging from $-0.3 \%$ to $+0.5 \%$. However, there are geographic limitations on where controlled blade bending could be viable, thus this area of innovation may not have the nation-wide impact needed to achieve deployment of supersized wind turbines in regions where they are most applicable. This approach could theoretically take advantage of the designed flexibility characteristics inherent in wind turbine blades, provided these loads can be accounted for in the blade design as well as the rail and road transport infrastructure. This innovation area offers an opportunity for additional study of blades, the rail system and trailers, and related infrastructure to further assess the viability, management of reaction loads caused by bending, and impact on blade fatigue life expectancy.

Segmented blades and on-site manufacturing pathways were found to increase system LCOE contribution, thus requiring greater savings in other turbine systems to achieve continued overall system LCOE reductions. Segmented blades offer features that other pathways are not currently able to provide, such as the ability to enable supersized blade deployment across the entire U.S. and blade joint solutions that are under active investigation and early deployment by major original equipment manufacturers. Other areas of innovation studied are further from market readiness. Additionally, there are likely opportunities to refine and optimize segmented blades to drive costs closer to a neutral LCOE impact, which for some regions, may be sufficient for wind deployment to be economically competitive. Segmented blades might become an optional feature available to the market along with monolithic blades and site-specific analysis would determine which option is most feasible and economically competitive.

Based on current labor-intensive blade manufacturing processes and methods, onsite (mobile) blade manufacturing faces economic challenges driven mainly by low tool/equipment utilization caused by time spent relocating and 
commissioning a mobile plant and elevated costs of local labor for hiring, training, plant commissioning, and first article manufacturing. These and other costs incurred each time the mobile plant is deployed represent significant challenges for any method of on-site blade manufacturing.

Figure 22 summarizes the results of our pathway assessment in terms of impact on LCOE, our current opinion of commercial readiness, and geographic breadth each pathway could offer.

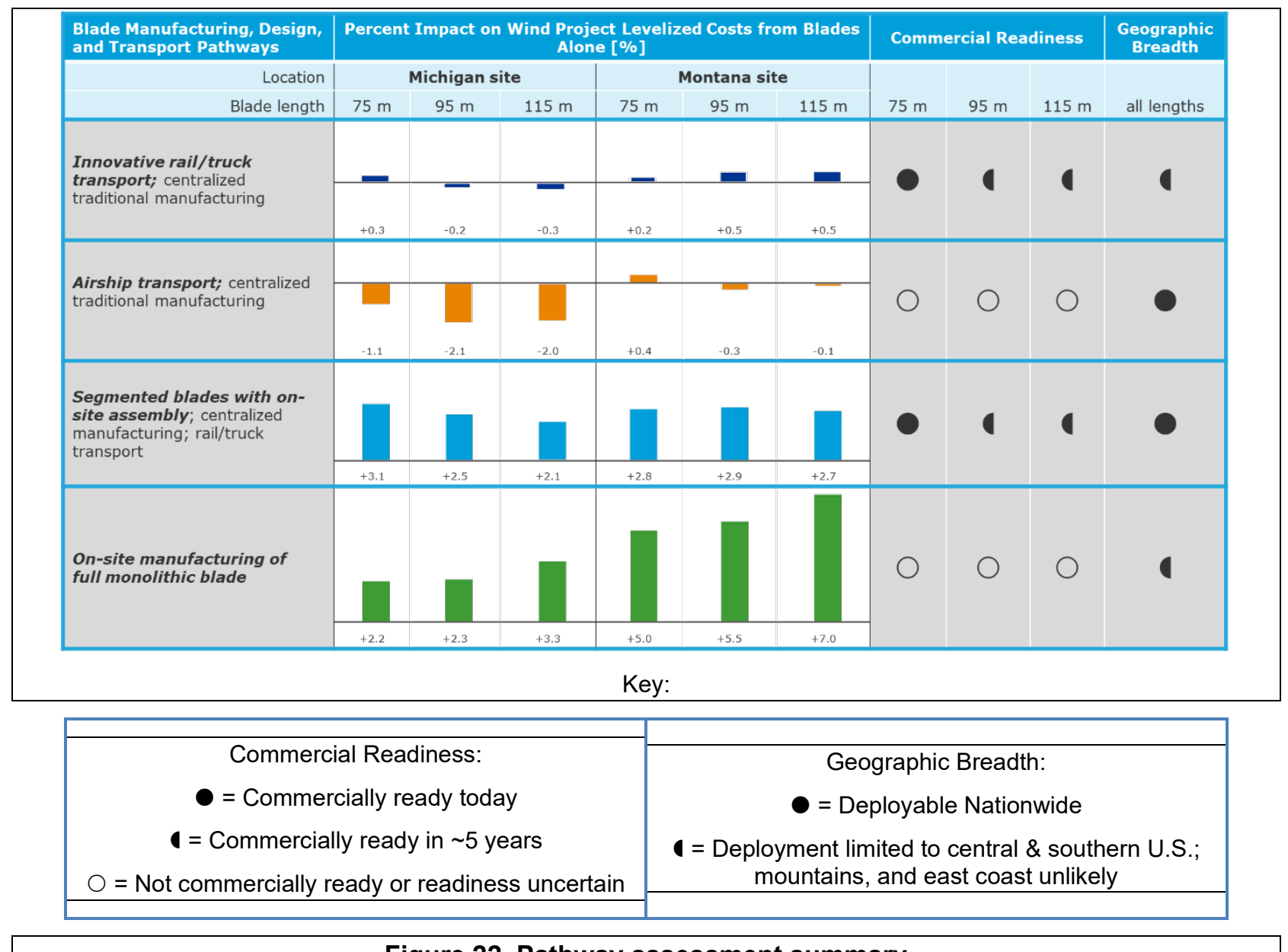

Figure 22. Pathway assessment summary

Based on the pathway analysis and findings, our industry understanding, and familiarity with U.S. DOE national laboratory core competence, we identified a number of high value R\&D topics that could be pursued to enable development of supersized blades. It is important to note that many R\&D topics are viewed as having benefits that could be applied to support some or all of the pathways studied. As mentioned previously, this project is not intended to "select a pathway", thus high-value R\&D topics are ones that have a significant impact across multiple pathways and leverage areas where U.S. DOE has strong competence, unique facilities, capacity to take high risks, and a long-term view.

Figure 23 presents DNV GL's identification of R\&D topics that could enable supersized blades. We cross-reference these topics to each innovation pathway and indicate our 
judgement on the degree of impact a given R\&D topic would have on enabling or addressing challenges in a given pathway. The pathways are ordered in terms of potential impact for lowering LCOE. Finally, we apply our judgement on DOE laboratories' ability to impact and advance the given R\&D topic.

\begin{tabular}{|c|c|c|c|c|}
\hline & \multicolumn{4}{|c|}{ R\&D Pathway Enabling } \\
\hline R\&D Topic & $\begin{array}{l}\text { 1. Innovative } \\
\text { transportation }\end{array}$ & $\begin{array}{l}\text { 2. Hybrid } \\
\text { solutions } \\
\text { (segmented } \\
\text { blades) }\end{array}$ & $\begin{array}{c}\text { 3. On-site } \\
\text { manufacturing }\end{array}$ & \begin{tabular}{|} 
Core DOE Lab \\
competence
\end{tabular} \\
\hline Aerodynamic design (lift-enhancing) & $\checkmark \checkmark \checkmark$ & $\checkmark \checkmark$ & $\checkmark$ & $\checkmark \checkmark$ \\
\hline Rotor configuration options (e.g., downwind) & $\checkmark \checkmark$ & $\checkmark$ & $\checkmark$ & $\checkmark \checkmark \checkmark$ \\
\hline Advanced aeroelastic modeling (dynamic stability, deflections) & $\checkmark \checkmark \checkmark$ & $\checkmark \checkmark$ & $\checkmark \checkmark$ & $\checkmark \checkmark \checkmark$ \\
\hline Advanced controls / sensor technologies & $\checkmark \checkmark \checkmark$ & $\checkmark \checkmark \checkmark$ & $\checkmark \checkmark$ & $\checkmark \checkmark \checkmark$ \\
\hline Blade leading-edge erosion & $\checkmark \checkmark \checkmark$ & $\checkmark \checkmark$ & $\checkmark \checkmark$ & $\checkmark$ \\
\hline Blade/rotor aeroacoustics & $\checkmark \checkmark \checkmark$ & $\checkmark \checkmark \checkmark$ & $\checkmark \checkmark$ & $\checkmark \checkmark$ \\
\hline High-stiffness / low-cost materials (e.g., industrial carbon fiber) & $\checkmark \checkmark \checkmark$ & $\checkmark \checkmark \checkmark$ & $\checkmark \checkmark \checkmark$ & $\checkmark \checkmark \checkmark$ \\
\hline Structural joint technology & & $\checkmark \checkmark \checkmark$ & & $\checkmark$ \\
\hline Thermoplastic materials (mechanical properties) & $\checkmark$ & $\checkmark \checkmark \checkmark$ & $\checkmark \checkmark$ & $\checkmark$ \\
\hline Thermoplastic materials (fabrication and joining) & $\checkmark$ & $\checkmark \checkmark \checkmark$ & $\checkmark \checkmark$ & $\checkmark$ \\
\hline Robotic fabrication (including additive manufacturing) & $\checkmark \checkmark$ & $\checkmark \checkmark$ & $\checkmark \checkmark \checkmark$ & $\checkmark \checkmark$ \\
\hline \multirow[t]{4}{*}{ High-capacity airship development } & $\checkmark \checkmark \checkmark$ & & & \\
\hline & & \multirow[b]{2}{*}{ LCOE Opportunity } & Key: & \\
\hline & & & $\checkmark \checkmark \checkmark$ & Strong impact \\
\hline & & High & $\begin{array}{c}\checkmark \checkmark \\
\checkmark\end{array}$ & $\begin{array}{l}\text { Moderate impact } \\
\text { Low impact }\end{array}$ \\
\hline \multicolumn{5}{|c|}{ Figure 23. R\&D topics to enable supersized blades } \\
\hline
\end{tabular}

Additional work was completed on the feasibility of transporting a highly flexible blade by rail. Following is an excerpt from "Innovative Rail Transport of a Supersized Land-Based Wind Turbine Blade" (Carron, Scott, and Bortolotti 2020):

This work presents a study on the transportability of 100-m long wind turbine blades across the U.S. rail system via controlled blade flexing. A conventional design, named BAR- $00^{14}$, is found to violate the transportation constraints and is found to be not transportable on rail. An alternative design, named BAR-01, is investigated in terms of clearance, curvatures, and reaction forces. The BAR-01 blade is assessed as having deployment potential along the main rail lines of the United States for horizontal curvatures up to 13 degrees. Vertical curvatures up to 2,000- $\mathrm{ft}$ radius are found not to be limiting.

Overall, the study concludes that within the U.S. land-based market, coordinated advancements in blade design and rail transport tooling have the potential to extend the deployment of one-piece blade lengths into the 100-m range. The current designs, however, push the current state of the art in terms of blade compliance, and an in-depth investigation into the aero-servo-elastic behavior of rail-transportable blades, such as increased torsional deformations and reduced flutter margins, is ongoing. The preliminary results are promising. Downwind rotor configurations are part of the analysis and will be compared to equivalent upwind configurations in terms of costs and performance. Advanced wind turbine system design and optimization processes that integrate rail

${ }^{14}$ BAR-00 and BAR-01 are referred to as BAR-UAG and BAR-DRG, respectively, in this document. 
logistic constraints and transport load cases directly into the blade design and certification process are suggested.

Future work should also include a detailed techno-economic analysis to assess the regional LCOE reduction potential of rail transport with blade flexing. The analysis should address rail transport costs, including tooling and load monitoring; rail line routing details; scalability; last mile delivery; fatigue life impact; and the costs associated with additional rail transport design load cases and blade certification. The optimized rotor technologies based on controlled blade flexing on the U.S. rail system should be compared, on a cost basis, to alternative solutions, such as segmentation and on-site manufacturing, to provide forward guidance on which technologies or combination of technologies can enable the largest LCOE reductions and market opportunities on a regional basis.

This work and the report from DNV GL helped the laboratory team sharpen the focus on R\&D in in Task 4 for highly flexible rail-transportable blades. 


\section{Task 3: Research-and-Development Opportunity Screening}

\subsection{Subtask 3.A: Development of Big Adaptive Rotor Innovation Concept Catalog}

The national laboratory team developed and evaluated a catalog of potential game-changing technologies to allow for the continued growth of land-based wind turbines in the United States. Following is an excerpt from the technical report on "Investigation of Innovative Rotor Concepts for the Big Adaptive Rotor Project" (Johnson et al. 2019) ${ }^{15}$ :

The national laboratory team, consisting of experts from the National Renewable Energy Laboratory (NREL) and Sandia National Laboratories (Sandia), conducted a literature review and developed a catalog of 16 innovative technology concepts that had the potential to enable very large land-based blades. The concepts considered are shown in Table 4. There is a large range of technology configurations that span from the more conventional to those with completely different topologies compared to modern commercial turbines.

\begin{tabular}{|l|l|l|l|l|}
\hline \multicolumn{4}{|c|}{ Table 4. BAR Concepts Broken Down by Innovation Category } \\
$\begin{array}{l}\text { Rotor } \\
\text { Topology }\end{array}$ & Orientation & \multicolumn{1}{|c|}{$\begin{array}{c}\text { Blade } \\
\text { Configuration }\end{array}$} & Blade Design Features & Hub Configuration \\
\hline $\begin{array}{l}\text { Three- } \\
\text { bladed }\end{array}$ & Upwind & $\begin{array}{l}\text { Scaled } \\
\text { conventional } \\
\text { blade }\end{array}$ & No advanced features & Normal hub \\
\hline $\begin{array}{l}\text { Two- } \\
\text { bladed }\end{array}$ & Downwind & $\begin{array}{l}\text { Slender, high } \\
\text { tip-speed-ratio } \\
\text { blades }\end{array}$ & Bi-wing blade & Large hub radius \\
\hline Dual-rotor & $\begin{array}{l}\text { Highly flexible } \\
\text { blades }\end{array}$ & $\begin{array}{l}\text { Segmented/modular } \\
\text { blades }\end{array}$ \\
\hline Multirotor & $\begin{array}{l}\text { Low-induction } \\
\text { rotor }\end{array}$ & $\begin{array}{l}\text { Inflatable blades } \\
\text { Wake- } \\
\text { optimized rotor }\end{array}$ & Variable coned rotor \\
\hline
\end{tabular}

\footnotetext{
${ }^{15}$ For citations in excerpt, please reference the original publication. Please note that some of the figure and table numbers are different between the original and what is reported here. This is for continuity of this document.
} 
The first row (in red) shows the current dominant configuration for modern commercial land-based wind turbine technology. The following lines show variations from the current configuration grouped into categories by design considerations. Some of the categories reflect fundamentally different topologies from the current dominant design configuration (e.g., the dual rotor or multirotor), while others are design features that could apply to numerous concepts (i.e., blade segmentation is compatible with machines of two or three blades with upwind or downwind orientations). For the purposes of isolating and exploring the potential of each innovation, all of the above concepts were treated separately. In the following subsections, each concept is described at a high level in terms of the concept definition as well as potential benefits and challenges of the technology from a BAR project perspective.

\subsection{Subtask 3.B: Information Gathering To Support Techno-Economic Analysis and Identification of Science and Engineering Challenges}

After the lab team identified the promising technologies from Subtask 3.A, a workshop was held to elicit expert feedback and evaluate the performance impacts and science and engineering challenges associated with the BAR concepts. A technical report was issued on this topic, "Investigation of Innovative Rotor Concepts for the Big Adaptive Rotor Project" (Johnson et al. 2019). Following are a summary and excerpts:

In all, 65 wind energy experts from the national laboratories, DOE, academia, and the wind industry reviewed and evaluated the concepts at the workshop. There was also a brainstorming session where concepts not in the catalog were considered for evaluation. The inflatable blade and two-bladed concepts were introduced during the brainstorming session. Participants were placed into breakout groups. Each group evaluated the potential benefits and challenges of about eight concepts. Not all groups evaluated the same concepts, but at least three groups evaluated each of the concepts in order to reduce the uncertainty in the results. Numerical scores were assigned to each metric category, comments on the relative strengths and weaknesses were collected, and the data were aggregated by the national laboratory team. A similar exercise was conducted internally by experts at NREL and Sandia.

During the workshop, participants were asked to first evaluate the BAR concepts according to their potential to impact various metrics associated with system cost and performance. Then, for the same concepts, the participants were asked to consider the science and engineering challenges that might limit the ability to realize the BAR concepts.

Through this evaluation process, the goal was to produce a qualitative understanding of the research and development $(\mathrm{R} \& \mathrm{D})$ pathways to the realization of the various technologies and the expected impact to system performance and costs if the concepts were successfully commercialized.

Participants were first asked to assess the potential of different BAR concepts to influence key wind plant (not just turbine) performance and cost metrics. The classic metric for evaluating an innovation from a full wind power plant perspective is in terms of the LCOE, which aggregates CAPEX (that include both turbine capital costs [TCC] 
and BOS costs) and OPEX (that include O\&M costs, financing [F] terms, and AEP) into a single equation.

$$
L C O E=\frac{F * C A P E X+O P E X}{A E P}
$$

While still critical to the competitiveness of wind energy compared to other electricity generation technologies, there is increasing emphasis on metrics of system performance other than LCOE. As the share of wind energy in the overall electricity generation portfolio in many regions grows, there is growing interest in the value that wind energy has to the overall electricity system in terms of:

- Energy value: the correlation of wind energy production with energy demand as well as its predictability and dispatchability to apply downward pressure on time-varying electricity prices

- Capacity value: the correlation of wind energy production with demand profiles over the course of the year so that there is less need to back up wind energy with alternative electricity generation sources

- Ancillary services value: the ability to support grid reliability and stability at various time scales.

Although different systems have different needs for wind energy depending on the generation mix of a specific electric grid system, in an increasing renewable-energydominated electricity system there will be increasing pressure on wind power plants to not only provide competitive LCOE but also increasing value in the above categories to the electric grid. However, for the purposes of BAR concept development, critical evaluation of these system value metrics is very difficult such that the value to the system was simplified to the potential of the concepts to increase CF, which would in turn support all the above system value metrics.

Beyond LCOE and system value, additional metrics that could be considered are the ability of the concept to minimize negative societal and environmental impacts as well as maximize workforce and economic development. These were not directly included in the evaluation process but may be part of the BAR concept evaluation later in the project.

The final metrics for evaluation of the technologies are provided in Table 5.

\begin{tabular}{|c|c|c|c|c|c|}
\hline \multicolumn{6}{|c|}{ Table 5. Cost and Performance Metrics for BAR Concept Evaluation } \\
\hline \multicolumn{5}{|c|}{ Levelized Cost of Energy } & $\begin{array}{l}\text { Economic } \\
\text { Value }\end{array}$ \\
\hline $\begin{array}{l}\text { Turbine } \\
\text { Capital Costs }\end{array}$ & $\begin{array}{l}\text { Turbine } \\
\text { Spacing }\end{array}$ & $\begin{array}{l}\text { Foundations/ } \\
\text { Transportation/Erection }\end{array}$ & $\begin{array}{l}\text { Annual Energy } \\
\text { Production }\end{array}$ & $\begin{array}{l}\text { Operational } \\
\text { Expenditures }\end{array}$ & Capacity Factor \\
\hline
\end{tabular}


challenges associated with the concepts (i.e., gaps in understanding in wind energy physics that would impact the concept). Participants were specifically asked to consider (1) What is new/novel about the concept that may challenge the current state-of-the-art in understanding wind turbine and plant physics? and (2) What about the operating environment of these machines is not well understood? Table 6 provides the categories of fundamental science that were considered for each BAR concept.

\begin{tabular}{|l|l|l|l|}
\hline \multicolumn{2}{|c|}{ Table 6. Science Challenges } \\
\hline
\end{tabular}

For each subcategory, reviewers were asked to evaluate whether the state-of-the-art physical models were appropriate to analyze the new concept. For blade aerodynamics, the capability of the state-of-the-art model to characterize unsteady aerodynamics for the new concept was assessed. For wakes, the capability of the state-of-the-art model to characterize wake merging or reenergization was assessed. For aeroelasticity, the capability of the state-of-the-art model to characterize things like flutter was assessed. For noise, the capability of the state-of-the-art model to characterize noise generation was assessed. For atmosphere, the capability of the state-of-the-art model to characterize inflow conditions at heights above $200 \mathrm{~m}$ was assessed.

While Table 6 focuses on gaps in understanding, it is necessary to characterize the engineering challenges associated with the various BAR concepts. Participants were asked to consider how BAR concept technology development would face various engineering challenges that may require $R \& D$ investment to overcome. Table 7 summarizes these categories.

\begin{tabular}{|c|c|c|c|c|c|c|c|}
\hline & Blade Design & & Controls & Manufacturing & Logistics & Reliability & $\begin{array}{l}\text { System } \\
\text { Impacts }\end{array}$ \\
\hline Materials & Aerodynamics & Structures & $\begin{array}{l}\text { Controls/ } \\
\text { Sensors }\end{array}$ & $\begin{array}{l}\text { Integration/ } \\
\text { Manufacturing }\end{array}$ & $\begin{array}{l}\text { Transport/ } \\
\text { Logistics/ } \\
\text { Installation }\end{array}$ & Reliability & $\begin{array}{l}\text { Rest of } \\
\text { System } \\
\text { Design }\end{array}$ \\
\hline
\end{tabular}

Again, in Table 7, many subcategories were considered. For each subcategory, reviewers were asked to evaluate whether the new concept presented any technology development challenges. For blade materials, reviewers considered the ability to make the necessary materials needed for the concept. Reviewers considered the ability to design airfoils and structures needed for the concept. Reviewers considered the ability to design unique controller and sensor requirements needed for the concept. They also considered any 
challenges with integration of the technology and/or manufacturing of the concept. Transportation logistics and installation were considered for each concept, understanding that there are physical size limits to transportation. Many of the novel concepts may impact reliability either positively or negatively. Reviewers also considered impacts to the rest of the system (e.g., tower, foundation, yaw system).

\subsection{Subtask 3.C: Big Adaptive Rotor Innovation Concept Initial Techno- Economic Analysis and Investigation of Science and Engineering Challenges}

After the workshop was completed, the national lab team analyzed the information collected from the blade design experts. To further quantify the data, the team averaged the scores for each category and between the different groups that evaluated the same concepts. Additionally, the national lab team evaluated each concept using the metrics shown in Table 5-Table 7. Figure 24 shows the average workshop results, internal lab results, and the average combined results for the cost and performance metric. Each concept was evaluated on a scale from 1-5, with 5 representing a positive effect on the cost and performance. Results show that the concepts on the left side of the chart are deemed to have a positive impact on the cost and performance.

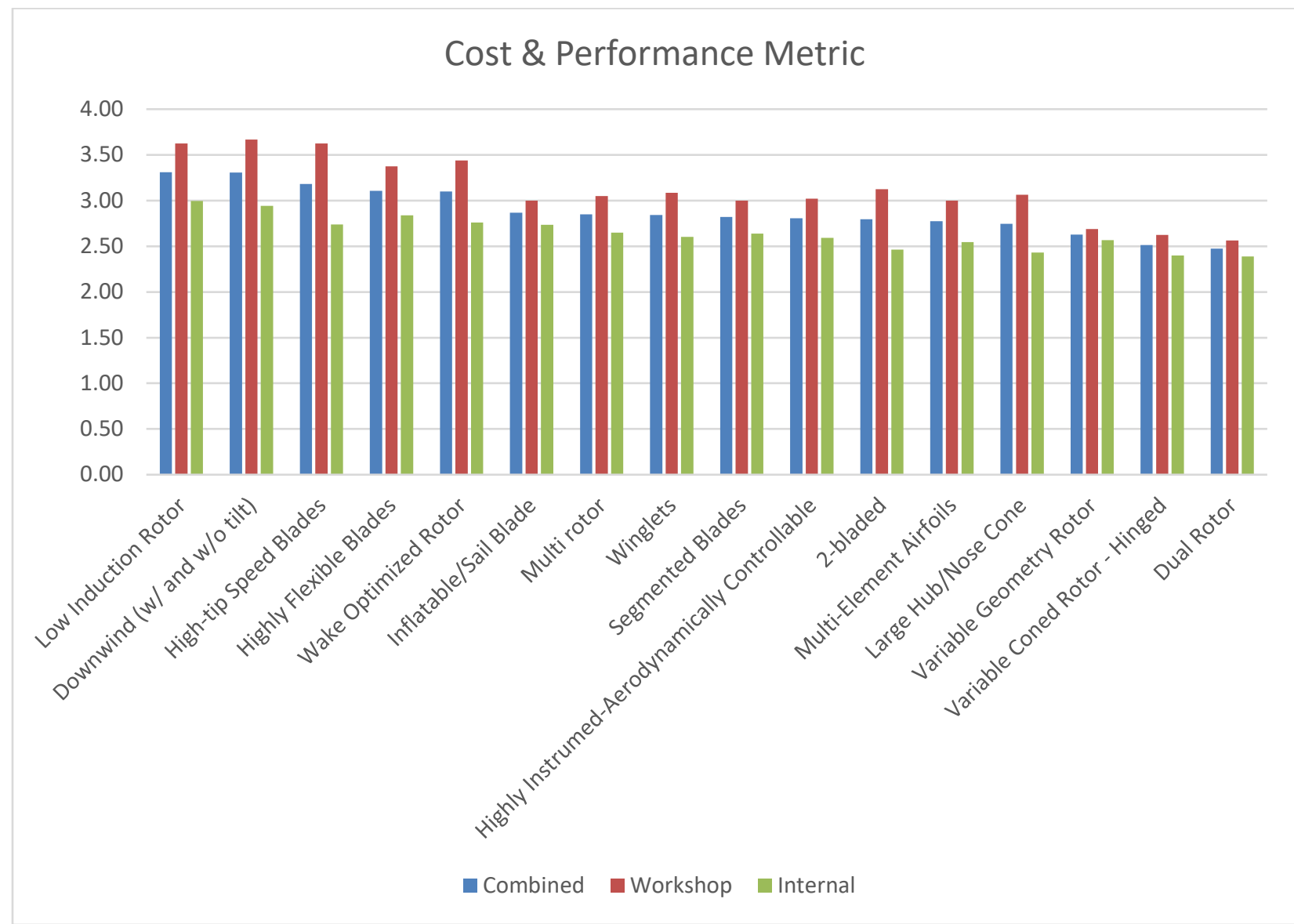

Figure 24. Performance metric for all concepts

For simplicity, the metrics from Table 6 and Table 7 were evaluated together, as shown in Figure 25 . Again, each concept was evaluated on a scale from 1-5, with 5 representing the least amount 
of science and engineering challenges associated with the concept. Results show that the concepts on the right side of the chart have the most open challenges and therefore are the ripest for R\&D.

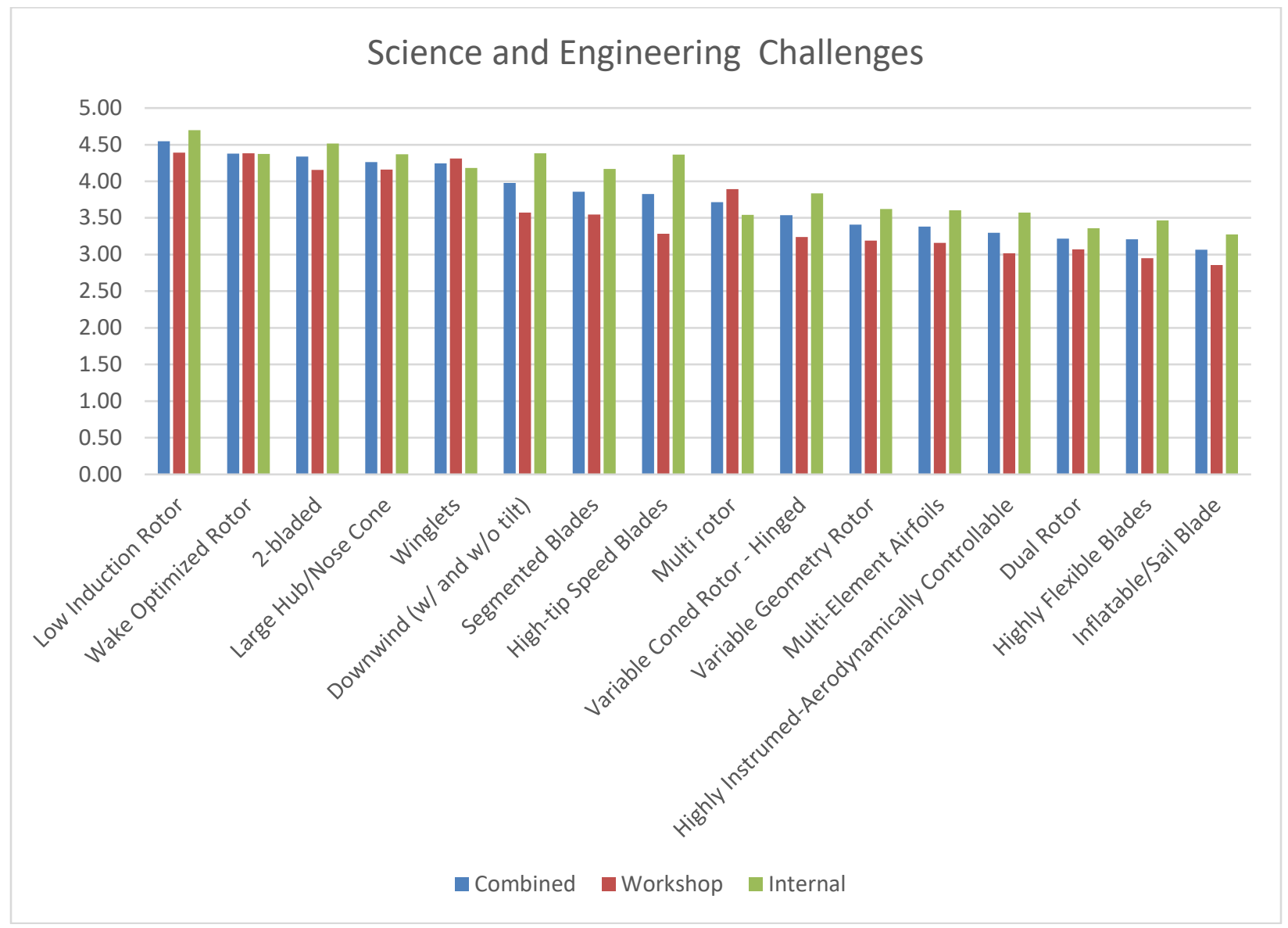

Figure 25. Science and engineering challenges

We established a combined metric to qualify the competing interests between capital cost and performance improvements with concepts that require investment in $\mathrm{R} \& \mathrm{D}$. The combined metric is defined as the cost and performance score divided by the science and engineering score. The combined metric gives the best insight into concepts that are both highly impactful and require R\&D investment (shown in Figure 26). 


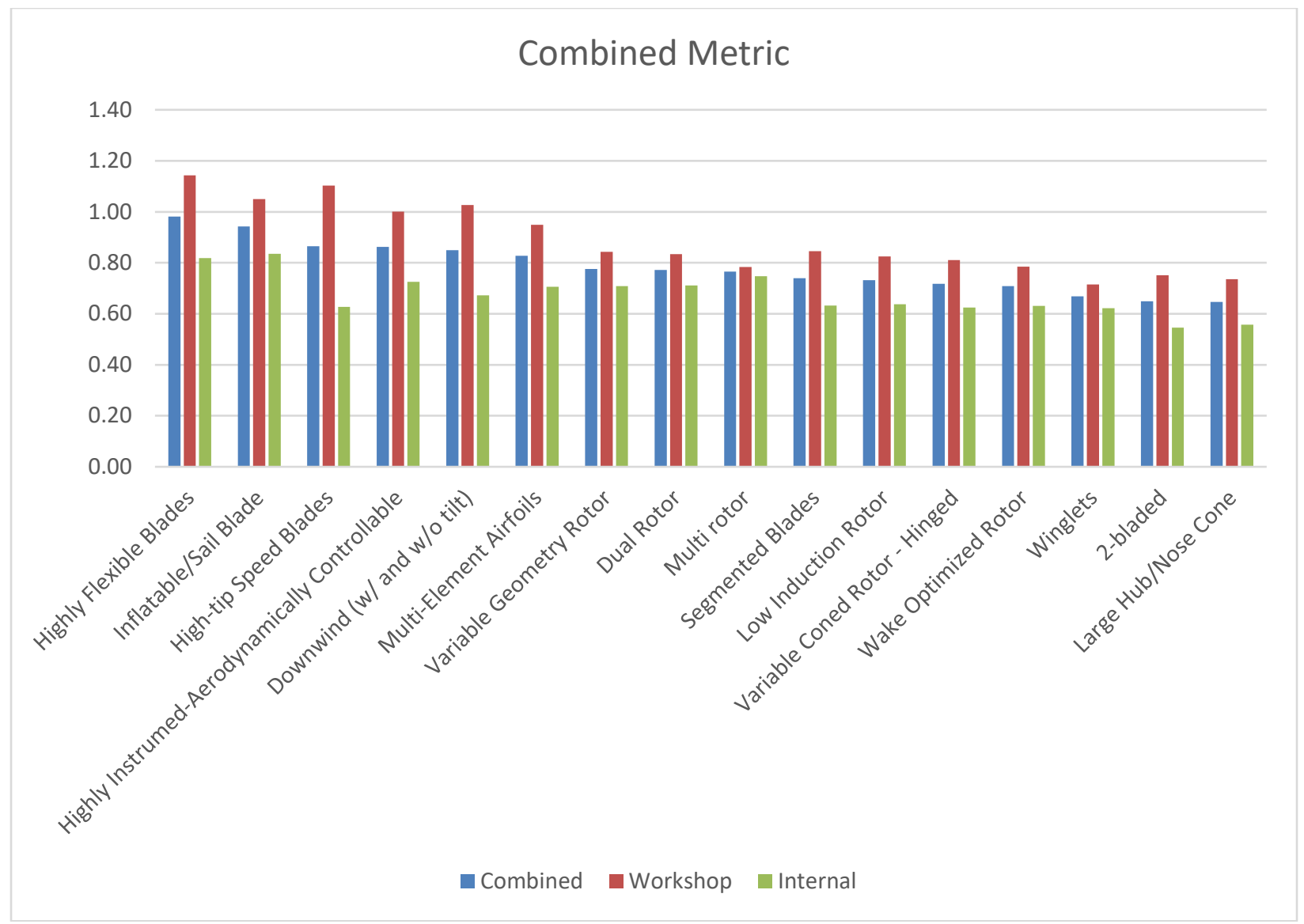

Figure 26. Combined metric performance

This method was a combination of a qualitative and a quantitative metric to determine the concepts for the down-selection. Based on this process, the following concepts were selected for continued analysis and detailed design: highly flexible rail-transportable blades, downwind rotors, DAC devices, inflatable blades, bi-wing blades, and five-bladed rotors. A detailed design of these concepts was completed in Task 4 and is summarized in Section 4. 


\section{Task 4: Detailed Design Studies}

This task started with a survey of the important science and engineering challenges and identification of modeling upgrades needed to enable the analysis and design of the innovative BAR concepts. After the models were updated and improved, the team completed detailed design of the BAR concepts to determine the feasibility of each concept.

\subsection{Subtask 4.A: Identification of Science and Engineering Challenges and Modeling Gaps}

Once the down-selection was completed in Task 3, the national lab team conducted a phenomena identification and ranking table (PIRT) analysis, as shown in Table 8. This analysis helped the team identify the area where further R\&D would be the most beneficial. The PIRT identifies the phenomena of importance for each concept and allowed the team to evaluate the ability to model the physics, the current code capability, and the level of model validation for the given phenomena based on a low-medium-high designation. The green, yellow, and red color coding is used to designate the acceptability between necessary model adequacy and the current estimated ability, with green noting acceptable and red noting unacceptable (Hills 2015). Finally, based on the prior analysis, a planning priority level is determined, with "high" being the top priority and "low" being the bottom priority. 
Table 8. PIRT

\begin{tabular}{|c|c|c|c|c|c|c|c|}
\hline \multirow{2}{*}{ Concept } & \multirow{2}{*}{ Challenge } & \multirow{2}{*}{ Phenomenon } & \multirow{2}{*}{$\begin{array}{c}\text { Importance at } \\
\text { Application } \\
\text { Level }\end{array}$} & \multicolumn{3}{|c|}{ Model Adequacy } & \multirow{2}{*}{$\begin{array}{c}\text { Planning } \\
\text { Priority }\end{array}$} \\
\hline & & & & Physics & Code & Validation & \\
\hline \multirow{5}{*}{ Downwind } & Tower shadow on passing blades & Tower shadow & High & High & Medium & Medium & Medium \\
\hline & Dynamic stall from incoming vortex & Unsteady aerodynamics & High & Medium & High & High & Low \\
\hline & Model flow for tilted rotors & Wake propagation & Low & Medium & Low & Low & Low \\
\hline & Controls; pitch to stall & Controls & Medium & Medium & Medium & Low & Medium \\
\hline & Noise & Aeroacoustic noise & High & Medium & Medium & Low & High \\
\hline \multirow{8}{*}{$\begin{array}{l}\text { Highly Flexible } \\
\text { Blades }\end{array}$} & Out-of-plane aerodynamics; high deflections and coning & Blade aerodynamics & Medium & Medium & Low & Low & High \\
\hline & Wake modeling of blades with large deflections & Blade/wake interactions & Low & Medium & Low & Low & Low \\
\hline & Get accurate Campbell diagrams and damping & Modal analysis of flexible blades & High & High & Medium & Medium & Medium \\
\hline & Changing cross-sectional geometry at the root & Structural response & Medium & High & Medium & Low & Low \\
\hline & High Reynolds number airfoil data & High Reynolds number & Medium & Unknown & High & Low & Low \\
\hline & Aeroelastic stability & Modal analysis & High & High & Medium & Low & Medium \\
\hline & Design of pitch system/actuator & Controls & High & High & Low & Medium & Medium \\
\hline & Higher noise rotor & Noise & High & Medium & Medium & low & Medium \\
\hline \multirow{2}{*}{$\begin{array}{l}\text { High-Tip-Speed- } \\
\text { Ratio Blades }\end{array}$} & Higher erosion & Material science & Medium & Medium & Low & Medium & Medium \\
\hline & Dynamic shape of airfoil & Unsteady aerodynamics & High & High & High & Medium & Low \\
\hline \multirow{5}{*}{$\begin{array}{c}\text { Distributed } \\
\text { Aerodynamics }\end{array}$} & Noise & Noise & High & Medium & Medium & low & Medium \\
\hline & Interaction with torsional degrees of freedom & Aeroelastics & Medium & High & Low & Low & Low \\
\hline & Aerodynamics of the flap; transients & Dynamic stall & Medium & Medium & Low & Low & High \\
\hline & Design of actuation system & Controls & High & High & Low & Medium & Medium \\
\hline & 3D effects & 3D aerodynamics & High & High & Low & Medium & Medium \\
\hline \multirow{3}{*}{ Bi-Wing Blade } & Noise from more sources & Noise & High & Medium & Medium & low & Medium \\
\hline & Structural analysis for more than one element & Structural response & High & High & Low & Low & High \\
\hline & Airfoil shape might change over time & Blade aerodynamics & High & High & High & High & Low \\
\hline Inflatable Blade & Optimization of decoupled aerodynamics/structure & Aeroelastics & High & High & High & Low & Medium \\
\hline
\end{tabular}


Based on the PIRT, the team also completed a modeling gaps analysis. This section presents the identified challenges, grouped per concept, excerpted from an internal NREL/DOE memo (Branlard, Bortolotti, and Jonkman 2019):

Downwind turbines have a series of science and engineering challenges:

- Blade aerodynamics: the understanding and the modeling of the tower shadow effects require improvements.

- Wake: the capability of highly tilted downwind rotors to capture energy from the flow above a wind farm has been numerically investigated, but it requires further research as well as a validation process.

- Noise: the generation of aeroacoustic noise in downwind turbines is one of the factors that have prevented the development of downwind machines in the past decades. Nonetheless, the acoustic emissions of very large downwind rotors are less clear. In addition, the numerical models to predict low-frequency noise have been used and validated far less than the models predicting noise in the acoustic range, with only a few studies available in the public domain.

- Structure: the benefits in structural design generated by the downwind orientation of the rotor should be better characterized with a detailed assessment.

- Reliability: the reliability of drive-train systems for downwind machines raised concerns among the workshops participants, and a suggestion for further investigation was provided.

Slender/high tip speed ratio/highly flexible blades call for improvements in the areas of aerodynamic modeling, wake characterization, aeroelasticity, noise, materials, airfoils, controls, and reliability:

- Blade aerodynamics: large out-of-plane deflections challenge some of the assumptions used by common aerodynamic models: actuator disk assumptions, radial independence of streamtubes. This problem is increased in the presence of highly coned rotors. Highly flexible blades may also increase the unsteadiness and the three-dimensional aerodynamic effects, possibly increasing the importance of dynamic stall models. In addition, for high tip speed blades compressibility effects start to have an impact on the aerodynamics, in particular in colder climate or lower air-density areas. Dedicated aerodynamic solvers or engineering models are required to assess the airfoil performances.

- Wake: with larger deflections of each individual blade, the near-wake may diverge from a uniform helical shape and the interaction between the individual blades and the local near wake may be increased. This will challenge the models that do not account for the position and dynamics of the near wake.

- Aeroelasticity: faster and more flexible rotors may complicate the dynamics of the machine, with more dangerous intersections between the natural frequencies and excitation frequencies.

- Noise: the generation of aeroacoustic noise is the actual factor currently limiting higher rotor velocities. Noise abating mechanisms should be a topic of research investigations.

- Materials: highly slender blades may require carbon fiber in some of the structural members, primarily the spar caps.

- Structures: structural models should be able to represent the large deflections that result from the increased flexibility of the blades. In particular, the proper account of material couplings is of increased importance as the deflections become larger. In addition, blades are often 
modeled via a combination of $2 \mathrm{D}^{16} \mathrm{FE}$ cross-sectional solvers (Variational Asymptotic Beam Sectional Analysis (VABS), Becas, ANisotropic Beam Analysis (ANBA), etc.) and beam models. This setup assumes no distortion of the cross-sectional shape. This assumption may lose validity for very flexible blades.

- Airfoils: faster rotating blades may accelerate erosion issues, which suggests the need for airfoils less sensitive to roughness and/or materials less susceptible to erosion. High tip speed blades would also operate at Reynolds number values above 10 million. Airfoil performances reported from wind tunnel measurements are usually obtained for Reynolds number between 1 and 6 million depending on the wind tunnel capacity. Further, CFD simulations at high Reynolds number are challenging, in part due to the difficulty of modelling the extremely thin boundary layer and determining the transition. The uncertainty on the airfoil performances is hence greater at high tip speeds and larger rotors.

- Controls: the ability to model the full pitch system including actuators is becoming more critical because of the size and weight of wind turbine blades. In addition, controllers may need to be capable of reacting faster than is currently possible on this rotor size and might need additional sensors.

- Reliability: faster rotating blades may require improved leading-edge protection to limit leading-edge erosion.

Distributed aerodynamic controls returned challenges in several disciplines, mostly blade aerodynamics, noise, controls, integration \& manufacturing and reliability.

- Aerodynamics: distributed aerodynamic controls consist of a large family of concepts, all with their specific characteristics. This being said, challenges in the understanding and modeling of the aerodynamic effects of the various concepts is certainly a major challenge. The unsteady aerodynamics of variable shape airfoil is a complex phenomenon. An accurate treatment is computationally expensive since it requires the resolution of the boundary layer and a simulation tool that can account for moving boundaries. Few engineering models and validation cases are available. They are limited to $2 \mathrm{D}$ and require tuning for different airfoil shapes.

- Noise: the nature of distributed aerodynamic controls is to perturb the flow along the wind turbine blade. This leads to potential benefits but may also easily cause undesired increments in the aeroacoustic noise.

- Controls: distributed devices on the blades require a description of the blades within the control system by several degrees of freedom, all potential controllable.

- Integration and manufacturing: blades become more complex to manufacture, transport and install.

- Reliability: distributed control devices have so far been limited in their deployment mostly because of concerns in their reliability, where the risk of incurring higher maintenance costs is real. Similarly, failure of part of a complex control system may pose serious hazards to the whole machine and should be investigated.

\footnotetext{
16 Two-dimensional (2D)
} 
Spanwise segmentation raises concerns in the fields of materials, structures, integration and manufacturing and reliability. Chordwise segmentation is instead a much more unexplored area, where the challenges are unknown.

- Materials: materials are key to keep the mass and the cost of the joint to a minimum.

- Integration and manufacturing: the largest challenges for blade segmentation consist in achieving a cost effective and reliable spanwise joint design.

Bi-wing blades have seldom been a topic of research and few workshop participants had a clear understanding of the concept and its potential benefits and challenges. This resulted in quite a scatter in the responses and large uncertainty:

- Aerodynamics: Blade element momentum (BEM) may lose accuracy and 3D CFD may rapidly become a necessity.

- Noise: the geometry of the structure may be beyond the validity range of existing noise models, especially in regard to trailing edge noise and the joint.

- Structures: the complex structures would most likely require a generic multi-body approach as well as 3D finite element model (FEM) studies. Concerns have been expressed in preserving torsional and shear stiffness.

- Installation, transport, reliability: the low technology readiness level (TRL) of the concept causes significant uncertainty in these fields.

Inflatable blades, similarly, to bi-wing blades, have so far been not well defined. Nonetheless, multiple challenges exist already at the preliminary level:

- Aerodynamics: unless the inflatable blades are filled with a very rigid (but then heavy) foam, airfoil shapes may be prone to flexibility of the outer membrane and of the filler. In that case, high-fidelity or engineering models will be required to capture the flexibility of the airfoil shape and account for the structural and aerodynamics effects. These effects may be captured via independent structural and aerodynamics models or using a fully coupled fluid-structure interaction (FSI) model, preferably in 3D.

- Noise: similarly, the noise generation may become much more complicated than in today's conventional blades.

- Materials: significant research in membrane technology may be needed.

- Structure: the structural and the aerodynamic designs may be uncoupled, with possible large benefits. This said, a detailed design optimization would be needed. In addition, the connection between the inflatable airfoils and the load carrying structure is to be conceived. Finally, inflatable blades are likely to suffer from a reduced torsional stiffness. In that case, flutter margins may be reduced.

- Integration and manufacturing: this is possibly the largest field of science and engineering challenges, with a high need of engineering studies required.

Four-to-five bladed rotors have not been evaluated in the workshops. A preliminary assessment of the challenges nonetheless returns the following issues:

- Aeroelasticity: most aeroelastic tools are not equipped to model more than three blades. Additionally, a higher number of blades would inherently be more flexible and subject to all the issues of highly flexible blades noted earlier.

- Structures: the hub design is complex and requires engineering studies. 
Next, a gaps analysis was completed based on phenomena. For out-of-plane aerodynamics, the BEM method relies on actuator-disk assumptions and hence should apply only to axisymmetric rotor loads contained in a plane. In practice, the assumptions have been relaxed, and the method has been successfully applied to small out-of-plane displacements and nonsymmetrical loads. Vorticity-based methods or other CFD methods, however, can more rigorously account for outof-plane aerodynamics. Because computational time is critical for aeroelastic design, computationally heavy options are considered only for validation purposes or for detailed design studies. Vortex code computational times might also become an issue, and speed-up techniques need to be addressed.

For distributed aerodynamic control devices, changes in airfoil shapes might occur if the airfoil structure is flexible or if active techniques are used to modify the airfoil shape (e.g., flaps). There is no dedicated implementation for flaps or other variable-shape airfoil aerodynamics in the OpenFAST tool set. Additionally, there is no control signal passed between the aerodynamic and servo module.

Noise modeling is relevant for the study of turbines at high-tip-speed ratios and for downwind turbines because of the aeroacoustic interaction with the tower. The recommendation is that noise models should also be integrated into the design procedures.

Structural modeling of the highly flexible blade is paramount. A nonlinear beam model, BeamDyn, is implemented in OpenFAST, but upgrades are necessary in the area of modal and stability analysis. These are very important features when designing highly flexible blades. We completed improvements for the lower fidelity structural models in the Wind-Plant Integrated System Design \& Engineering Model (WISDEM ${ }^{\circledR}$ ). To get the cross-sectional properties for the nonlinear beam model, a commercial code was necessary; however, there are open-source options - for example, Structural Optimization aNd AeroelasTic Analysis (SONATA) and ANBA4. ANBA4 is a 2-D cross-sectional solver, SONATA parameterizes the design inputs, and postprocesses and visualizes the results. We incorporated these models into the open-source framework of WISDEM to allow for slightly higher fidelity optimization and analysis of composite structures. Finally, improvements are necessary for the Numerical Manufacturing And Design Tool (NuMAD) 3D FEM code, specifically in the ability to optimize blade designs. Efforts will be made to improve the optimization functionality of the code.

\subsection{Subtask 4.B: Model Development and Integration}

Based on the findings from Subtask 4.A, the national lab team implemented the following modeling upgrades to enable the analysis of the BAR innovation concepts.

- Slender highly flexible blade:

- Vortex model for improved aerodynamic analysis

○ Structural improvements for WISDEM

- Structural improvements for NuMAD

○ Aeroacoustics model for high-tip-speed-ratio blades

- Downwind: 
○ Nacelle blockage

- Active aerodynamic devices:

- Implemented active aerodynamic modeling capability in the aerodynamic and controls modules of OpenFAST.

\subsection{1 cOnvecting LAgrangian Filaments}

cOnvecting LAgrangian Filaments (OLAF) is a free vortex wake module used to compute the aerodynamic forces on moving two- or three-bladed horizontal-axis wind turbines. This module has been incorporated into the NREL physics-based engineering tool OpenFAST, which solves the aero-hydro-servo-elastic dynamics of individual wind turbines. OLAF is incorporated into the OpenFAST module AeroDyn v15 as an alternative to the traditional BEM option. Incorporating the OLAF module into OpenFAST allows for the modeling of highly flexible turbine blades along with the aero-hydro-servo-elastic response capabilities of OpenFAST.

The OLAF module uses a lifting-line representation of the blades, which is characterized by a distribution of bound circulation. The spatial and time variation of the bound circulation results in free vorticity being emitted in the wake. OLAF solves for the turbine wake in a time-accurate manner, which allows the vortices to convect, stretch, and diffuse. The OLAF model is based on a Lagrangian approach, in which the turbine wake is discretized into Lagrangian markers. There are many methods of representing the wake with Lagrangian markers. In this work, we used a hybrid lattice/filament method, as depicted in Figure 27. Here, the position of the Lagrangian markers is defined in terms of wake age, $\zeta$, and azimuthal position, $\psi$. A lattice method is used in the near wake of the blade. The near wake spans a user-specified angle or distance for nonrotating cases. After the near-wake region, the wake is assumed to instantaneously roll up into a tip vortex and a root vortex, which are assumed to be the most dominant features for the remainder of the wake. Each Lagrangian marker is connected to adjacent markers by straight-line vortex filaments, approximated to second-order accuracy. The wake is discretized based on the spanwise location of the blade sections and a specified time. After an optional initialization period, the wake is allowed to move and distort, thus changing its structure as the markers are convected downstream. To limit computational expense, the root and tip vortices are truncated after a specified distance downstream from the turbine. 


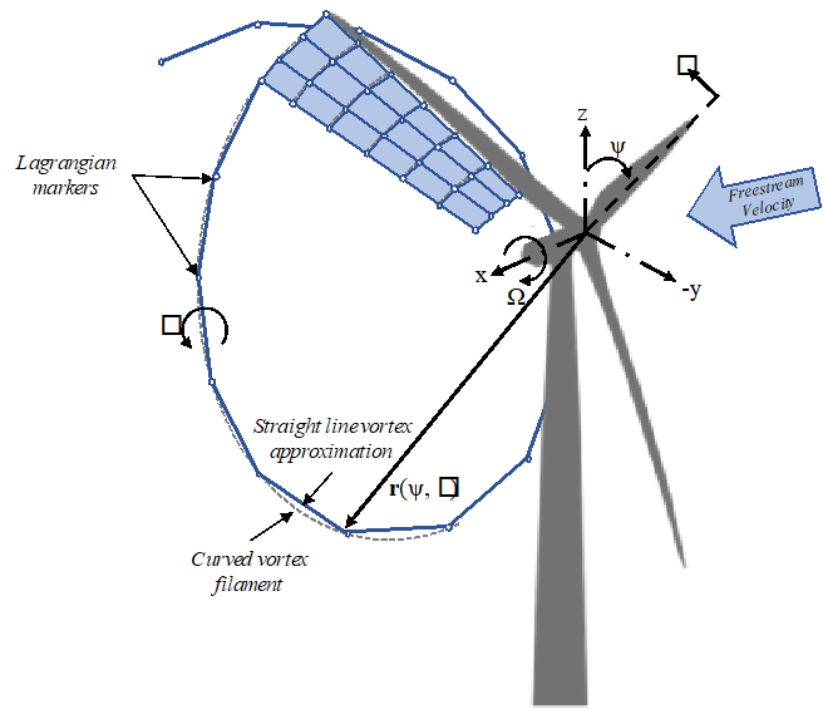

Figure 27. Evolution of near-wake lattice, blade-tip vortex, and Lagrangian markers. Image credit: OLAF User's Guide and Theory Manual (Shaler, Branlard, Platt 2020)

As part of OpenFAST, induced velocities at the lifting line/blade are transferred to AeroDyn v15 and used to compute the effective blade angle of attack at each blade section, which is then used to compute the aerodynamic forces on the blades. The OLAF method returns the same information as the BEM method, but it allows for more accurate calculations in areas where BEM assumptions are violated. Further details on the OLAF theory and implementation can be found in the OLAF User's Guide and Theory Manual (Shaler, Branlard, Platt 2020).

\subsubsection{WISDEM}

NREL has developed the systems engineering framework WISDEM for more than a decade, which now has a level of maturity that allows users to conduct a wide portfolio of investigations into wind turbine design. WISDEM is freely available online, ${ }^{17}$ and it comes with online documentation that is regularly updated ${ }^{18}$ and that contains a list of all the publications that are used in WISDEM. Throughout the duration of the BAR project, WISDEM has been extensively exercised and improved, from release 1.0 to the latest release, 3.2.0.

WISDEM implements the solver PreComp (Bir 2006), which offers the attractive advantages of being quick and simple to use, but it is known to be less accurate than commercial, state-of-theart, cross-sectional solvers, such as VABS (Chen, Yu, \& Capellaro, 2010), and it lacks the ability to estimate the shear stiffnesses (it populates only the 4-by-4 stiffness matrix). The geometrically exact beam model BeamDyn (Wang et al. 2017) implemented in OpenFAST requires the full matrices, and to overcome this limitation, we adopted and improved the open-source Python framework SONATA (Feil et al. 2020a). SONATA was originally developed at the Institute of Helicopter Technology at the Technical University of Munich, Germany, and performs the 2D

\footnotetext{
${ }^{17}$ WISDEM GitHub repository: https://github.com/WISDEM/WISDEM
}

${ }^{18}$ WISDEM documentation: https://wisdem.readthedocs.io/en/master/ 
meshing of arbitrary composite cross sections. Although it was originally conceived for helicopter applications, it has now been updated to support wind turbine blades.

Another crucial development of SONATA that happened under BAR involved coupling SONATA, which was originally coupled only to VABS, to the open-source cross-sectional tool ANBA4. A study then compared the ANBA4 and VABS codes and validated the outputs for some canonical beam experiments available in the literature. Both ANBA4 and VABS are capable of fully populating the 6-by-6 stiffness and inertia matrices, which are then used by the geometrically exact beam model, BeamDyn.

\subsubsection{NUMAD}

Within the BAR project, we upgraded NuMAD from release 2.0 to release 3.0. NuMAD 3.0 allows users to conduct detailed structural analysis of wind turbine blades and minimize blade mass through optimization. The user experience in NuMAD 2.0 was entirely dependent on a graphical user interface. The graphical user interface prevented the automation required in optimization routines. NuMAD 3.0 includes an object-oriented approach to manage the data structures for wind turbine blades. Although analysis of tip deflection, buckling, material rupture, total mass, and frequencies were possible in NuMAD 2.0, the new version automates the transfer of data from ANSYS back to NuMAD for the optimizer. In addition, two entirely new analysis procedures were developed for NuMAD during the BAR project. We developed a technique to determine the design loads. Increasing the scope of the structural aspects of the analysis required simplification from the loads; thus, the thousands of section forces and moments calculated during the dynamic structural analyses required in the system-level optimization were reduced to nine critical load cases. The other newly developed analysis procedure allowed users to evaluate fatigue damage for every material layer at various cross sections of a blade. Finally, we made compatible with the input/output YAML input file adopted by WISDEM, an implementation that allowed a quick turnaround of the design iterations between the two frameworks, improving the quality of the analysis.

\subsubsection{Aeroacoustics}

The BAR team coupled the latest release of OpenFAST with aeroacoustics models. NREL has decades of experience with the aeroacoustics emission of wind turbines, but the numerical models used to predict the noise emissions of the blades had never been formally coupled to the aeroelastic models. Within BAR, the models that originally belonged to NAFNoise and that were once poorly linked to FAST v7 have been cleaned, commented, documented, and improved. The results of the work are documented in (Bortolotti et al. 2020). The new code is now fully integrated into the OpenFAST GitHub repository. ${ }^{19}$ Recently, the models have been the subject of verification studies conducted within the International Energy Agency (IEA) Wind Task 39 on Quiet Wind Turbine Technology and validation studies conducted within the Aeroacoustics Assessment project supported by DOE at NREL (Bortolotti et al. 2021a).

\footnotetext{
${ }^{19}$ OpenFAST GitHub repository: https://github.com/OpenFAST/openfast
} 


\subsubsection{Distributed Aerodynamic Controls}

The fourth area of model development comprised supporting the numerical simulations of distributed aerodynamic control devices in OpenFAST and WISDEM. In OpenFAST, we updated the AeroDyn module to allow for the input of more than one set of airfoil polar data for a given flap angle or other condition for an arbitrary DAC device. This approach enables the use of multiple flap angles within a single simulation by interpolating polar data at given points, so not every discreet point must be given. This work was completed by Bonnie Jonkman at Envision Energy, under an industry partnership. Until this point, we assumed the aerodynamic polars to be known from some outside source; however, polars for the profiles that are being used with flaps being deflected in both the positive and negative directions were unavailable. Further, in a codesign situation, the profile and flap geometries might need to change; thus, within the WISDEM framework, a method is needed to calculate the aerodynamic polars for arbitrary geometries. This was done by creating a Python wrapper code that could call XFOIL to calculate the polars based on the interpolated base geometries. We also used XFOIL to obtain the profile geometries with flaps. This capability to create profile coordinates with flaps and run XFOIL to obtain aerodynamic polars for arbitrary geometries was added to the CCAirfoil instance within CCBlade. Finally, we modified the AeroelasticSE model to read and write the AeroDyn polar files that contain multiple polars for each blade station where a flap is located (as defined by the blade input file). Only stations where flaps are present will call the CCAirfoil instances to create the flap polars. If the flaps are not present at a given blade station, we used the interpolated polars from the blade input file.

\subsection{Subtask 4.C: Detailed Design for Big Adaptive Rotor Innovation Concepts}

After the modeling capability was upgraded, the NREL and Sandia National Laboratories (Sandia) teams conducted design studies of the flexible rail-transportable rotor concept (BAR designs), inflatable blade, the bi-wing blade, five-bladed rotors, and blades with distributed aerodynamic controls.

\subsubsection{Big Adaptive Rotor Designs}

The next subsections summarize the systems-level design analysis conducted at NREL and the detailed structural design optimizations conducted at Sandia. Five designs were developed for the project:

1. BAR-UAG (upwind - air transport - glass-fiber spar caps). This design is a baseline upwind configuration with no constraints on the maximum chord and represents a scaledup version of commercially available blades. The resulting design has a maximum chord of $5.3 \mathrm{~m}$, which would prevent transporting the blade on roads or rails and thus assumes an air transportation method that does not currently exist. A longer chord would marginally improve aerodynamic performance but would also incur increased manufacturing costs, as predicted by (Bortolotti et al. 2019). The diameter at the blade root was assumed to be equal to $4.5 \%$ of the blade length, which is $4.5 \mathrm{~m}$. The blade has a prebend of $4 \mathrm{~m}$ at the blade tip. This value is compatible with standard manufacturing limits. The blade adopts a conventional, glass-fiber, unidirectional laminate in the spar caps. 
2. BAR-DRG (downwind - rail transport - glass-fiber spar caps). This design has a flapwise stiffness that meets the requirements for controlled bending during rail transportation (Carron, Scott, and Bortolotti 2020). The spar caps are made of glass fiber and are thinner than in BAR-UAG. We reduced the chord to decrease the absolute thickness of the blade and to comply with a maximum value of $4.75 \mathrm{~m}$. Finally, we removed the prebend to relax the transportation constraints. The flexible blades are mounted in a downwind configuration to prevent tower strikes while keeping conventional values of rotor precone and nacelle uptilt angles. The blade deflections toward the tower are further mitigated with two changes to the conventional control strategy of upwind rotors. First, we reduced the cut-out wind speed from $25 \mathrm{~m} \mathrm{~s}^{-1}$ to $19 \mathrm{~m}$ $\mathrm{s}^{-1}$. Second, we switched the shutdown maneuvers below rated wind speed from standard pitch to feather to pitch to stall. The simulations conducted in OpenFAST showed that such a solution causes limited or no increase to the blade loads while changing the direction of the blade deflections, which now happens away from the tower because of the simultaneous increase in drag and lift during the shutdown.

3. BAR-DRC (downwind - rail transport - carbon-fiber spar caps). This design meets the same requirements of BAR-DRG, adopting the industry-baseline, pultruded carbonfiber laminate in the spar caps. The stiffer spar caps impose even thinner spar caps, which are no longer driven by ultimate tip deflection but by the buckling constraint. To meet the blade flapwise stiffness dictated by rail transportation requirements, the optimizer reduced blade chord and moved the airfoils inboard, effectively reducing both relative and absolute thickness along the span. The BAR-DRC rotor adopted the same control logic of BAR-DRG.

4. BAR-USC (upwind - segmented - carbon-fiber spar caps). This design aimed to represent a 100-m blade designed with today's technology. The design mounted a mechanical joint at a span location of $70 \mathrm{~m}$. The joint was assumed to weigh 2 tons and to cost $\$ 50,000$. The BAR-USC blade adopted the same chord and prebend of BAR-UAG, with the exception that the chord was limited to $4.75 \mathrm{~m}$ to allow for rail and blade transportation. We chose fairly small values of rotor cone, 4 degrees, and nacelle uptilt angle, 2 degrees, to obtain high power performance.

5. BAR-URC (upwind - rail transport - carbon-fiber spar caps). The last design adopted the same blade outer shape of BAR-DRC, but the blades were mounted in an upwind configuration. To prevent tower strikes, the rotor cone was set to 4 degrees and the nacelle uptilt to 8 degrees.

Readers interested in more details are invited to read the following two papers: "Land-Based Wind Turbines with Flexible Rail Transportable Blades - Part I: Conceptual Design and Aeroservoelastic Performance" (Bortolotti et al. 2021b) and "Part II: 3D FEM Design Optimization of the Rotor Blades," (Camarena et al. 2021) submitted to Wind Energy Science in the first half of 2021. The next subsections summarize the design approaches discussed in the two journal articles and provide a more technical look at the five wind turbine designs.

\subsubsection{Wind Turbine Systems Analysis}

Within the BAR project, we used WISDEM to design the wind turbine concepts at a preliminary level, with a special focus on the rotor. The rotor design optimizations parametrized the blade chord, blade twist, airfoil positions along the span, and spar cap thickness to minimize the LCOE. The constraints limited the ultimate strains in the spar caps to 3,500 microstrain and, for 
the upwind configurations, the maximum blade tip deflection. In addition, a novel model to simulate the rail transportation of blades via controlled bending added a constraint on blade flapwise stiffness (Carron, Scott, and Bortolotti 2020). A separate optimization sized the tower for the baseline configuration, whereas the recently updated drivetrain model was used iteratively within the design loop to have a preliminary sizing of the geared drivetrain configuration for all designs. At the end of the workflow, WISDEM called a turbine capital cost model, a detailed blade cost model (Bortolotti et al. 2019), and a finance cost model that led to the LCOE (Stehly \& Beiter, 2019). The blue block in Figure 28 represents this workflow, which was built based on the optimization library OpenMDAO (Gray et al. 2019).

Once WISDEM converged to a solution, we used its outputs to feed four other numerical frameworks, as illustrated in Figure 28. First, we tuned the dynamic controller Reference OpenSource Controller (ROSCO) for the aeroservoelastic simulations for each design iteration of the five wind turbines.

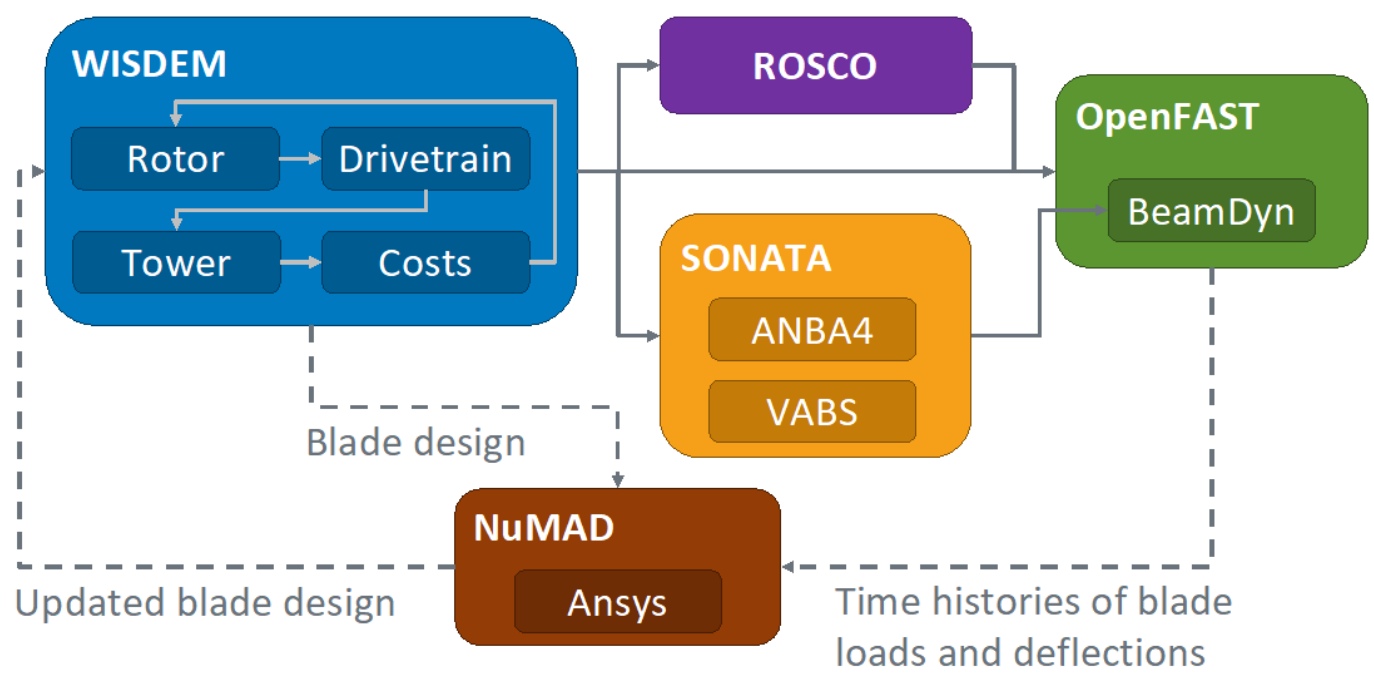

Figure 28. The design workflow adopted to develop the flexible rail-transportable BAR rotors ROSCO is an open-source controller and is available on GitHub. ${ }^{20}$ The controller can be tuned using a Python-based library that contains automated processes to generate proportional-integral gains for the generator torque and blade pitch controllers. In parallel, we loaded the blade design into the novel cross-sectional analysis framework SONATA (Feil et al. 2020b) which generated the BeamDyn models of the blades. Together with the BeamDyn files generated by SONATA, we automatically generated the rest of the OpenFAST model with the newly developed framework, called Wind Energy with Integrated Servo-control (WEIS). ${ }^{21}$ Within WEIS, we ran aeroservoelastic simulations covering normal operation, coherent gusts, shutdowns, and storm events per IEC-61400-1. We ran the design load cases 1.1, 1.3, 1.4, 1.5, 5.1, 6.1, and 6.3 with six 10-min. turbulent wind speeds using the Eagle, DOE's high-performance computing system.

${ }^{20}$ ROSCO GitHub repository: https://github.com/NREL/ROSCO

${ }^{21}$ WEIS GitHub repository: https://github.com/WISDEM/WEIS 
Further, we adjusted the rotor precone angle and the nacelle uptilt angles to ensure that the deflections estimated by BeamDyn did not violate the tower clearance constraint. Notably, the deflections estimated by WISDEM were always close enough that the corrections in cone and tilt angles did not exceed 1 degree.

As a last step of the iterative design workflow illustrated in Figure 28, the rotor blade model generated by WISDEM together with the time histories of loads and deflections computed by OpenFAST were passed to NuMAD, which is the blade structural optimization framework developed at Sandia. The next subsection elaborates on the design approach adopted by NuMAD and how the model was fed back into WISDEM for a new iteration.

\subsubsection{Detailed Structural Optimization of the Rotor Blades}

The system design studies conducted at NREL assumed two major simplifications in the structural analysis and design of the wind turbine blades. First, NREL optimized blade structures only in terms of spar cap thickness, with all other elements of the blade structure assumed a priori, starting from the IEA Wind Task 37 10-MW reference wind turbine (Bortolotti 2019). Second, the design constraints checked only that the maximum blade deflections and maximum spar cap strains were within the maximum allowable values. Such simplifications are acceptable in a preliminary phase but must be complemented with a separate structural optimization at higher fidelity. This step was performed at Sandia to ensure that the resulting blades in this study are feasible in all their components and to properly account for all design drivers.

Sandia performed detailed structural optimization of the NuMAD model in MATLAB by adopting the built-in function fmincon, a general nonlinear, gradient-based, optimization solver. The objective function was defined to be the total blade mass, with penalty terms added to enforce the constraints on maximum tip deflection, material rupture, buckling stability, fatigue damage, and natural frequencies in the flap and edge directions. At each design state in the optimization, the optimizer called upon NuMAD and the ANSYS finite element shell model to evaluate the state of these performance constraints under the appropriate loading conditions and form the total objective value for that design state. The gradient of the objective function was obtained with central differencing.

We optimized the model by modifying the thicknesses of the main blade layers/components along the spanwise length as well as the nominal width of the spar caps and extent of the shear webs. The outer shape and aerodynamic properties of each blade were not allowed to be modified in this process and were optimized only in WISDEM. We defined design variables to represent the thickness of each blade component at six evenly distributed points along the span, with the thicknesses of the intermediate points defined with spline interpolation. The thickness distribution of each component for the optimized blade designs is given in the next section.

Increasing the fidelity from the NREL beam model to the Sandia shell model required an increased amount of material properties. Table 9 and Table 10 list the required material properties for the NuMAD optimization. A difficulty encountered is that nine elastic properties and seven rupture properties are required for each of the five composite materials. Only the uniaxial glass composite had a set of properties completely defined by physical experiments. The rest of the properties had to be determined by analytical and numerical homogenization schemes. 
The BAR blades adopted standard unidirectional, biaxial, and triaxial glass-fiber laminates and a regular, aerospace-type, pultruded carbon fiber, referred to as industry standard. Within the BAR project, the team investigated an alternative lower-cost pultruded carbon-fiber laminate (Ennis et al. 2019).

Table 9. Mechanical Properties of Composite Laminates by Fiber Type. All Strength Properties Are Design Values (Not the Characteristic Values) and Are Obtained With $\gamma_{\sigma}=1.74$.

\begin{tabular}{|c|c|c|c|c|c|}
\hline Fiber Type & $\begin{array}{l}\text { Uniaxial } \\
\text { Glass }\end{array}$ & $\begin{array}{l}\text { Biaxial } \\
\text { Glass }\end{array}$ & $\begin{array}{l}\text { Triaxial } \\
\text { Glass }\end{array}$ & $\begin{array}{l}\text { Uniaxial Industry- } \\
\text { Standard Carbon }\end{array}$ & $\begin{array}{l}\text { Uniaxial Heavy- } \\
\text { Tow Carbon }\end{array}$ \\
\hline$E_{1}^{*}[\mathrm{GPa}]$ & 43.70 & 11.02 & 28.21 & 157.6 & 160.6 \\
\hline$E_{2}^{*}[\mathrm{GPa}]$ & 16.50 & 11.02 & 16.24 & $9.1^{a}$ & $9.1^{\mathrm{a}}$ \\
\hline$E_{3}^{*}[\mathrm{GPa}]$ & 15.45 & 16.05 & 15.84 & $9.1^{\mathrm{a}}$ & $9.1^{\mathrm{a}}$ \\
\hline$G_{12}^{*}[\mathrm{GPa}]$ & 3.265 & 13.23 & 8.248 & 4.131 & 4.131 \\
\hline$G_{13}^{*}[\mathrm{GPa}]$ & 3.495 & 3.488 & 3.491 & 4.131 & 4.131 \\
\hline$G_{23}^{*}[\mathrm{GPa}]$ & 3.480 & 3.488 & 3.491 & 2.689 & 2.689 \\
\hline$v_{12}^{*}[]$ & 0.262 & 0.6881 & 0.4975 & 0.3133 & 0.3133 \\
\hline$v_{13}^{*}[]$ & 0.264 & 0.1172 & 0.1809 & 0.3133 & 0.3133 \\
\hline $\boldsymbol{v}_{23}^{*}[]$ & 0.35 & 0.1172 & 0.2748 & 0.4707 & 0.4707 \\
\hline$\gamma_{\sigma}^{-1} X[\mathrm{MPa}]$ & 640.23 & 46.21 & 435.63 & 1285 & 772.7 \\
\hline$\gamma_{\sigma}^{-1} X^{\prime}[\mathrm{MPa}]$ & -370.7 & -70.69 & -343.1 & -878.2 & -673.5 \\
\hline$\gamma_{\sigma}^{-1} Y[\mathrm{MPa}]$ & 38.1 & 46.21 & 76.44 & $38.1^{\mathrm{b}}$ & $38.1^{\mathrm{b}}$ \\
\hline$\gamma_{\sigma}^{-1} Y^{\prime}[\mathrm{MPa}]$ & -82.18 & -70.69 & -174.7 & $-82.18^{b}$ & $-82.18^{b}$ \\
\hline$\gamma_{\sigma}^{-1} S[\mathrm{MPa}]$ & 30.17 & 124.5 & 85.06 & $30.17^{b}$ & $30.17^{b}$ \\
\hline$\gamma_{\sigma}^{-1} R[\mathrm{MPa}]$ & 18.97 & $18.97^{b}$ & $18.97^{b}$ & $18.97^{b}$ & $18.97^{b}$ \\
\hline$\gamma_{\sigma}^{-1} T[\mathrm{MPa}]$ & 6.21 & $6.21^{b}$ & $6.21^{b}$ & $6.21^{\mathrm{b}}$ & $6.21^{b}$ \\
\hline $\boldsymbol{m}[\mathrm{]}$ & 10 & 10 & 10 & 16.1 & 45.4 \\
\hline$\rho\left[\mathrm{kg} \mathrm{m}^{-3}\right]$ & 1940 & 1940 & 1940 & 1600 & 1600 \\
\hline
\end{tabular}

${ }^{a}$ Test result from a smaller fiber volume fraction. (Industry baseline pultrusion tests at $62 \%$ fiber volume fraction [Miller et al. 2019])

b Unidirectional glass composite value

Table 10. Mechanical Properties of the Isotropic Constituents. All Strength Properties Are Design Values (Not the Characteristic Values) and Are Obtained With $\gamma_{\sigma}=1.92$ (A larger $\gamma_{\sigma}$ is required by IEC (IEC-61400-1, 2005) for the foam.)

\begin{tabular}{|l|c|c|c|c|c|c|}
\hline & $\begin{array}{l}E \\
{[\mathrm{GPa}]}\end{array}$ & $v$ & $\begin{array}{l}\gamma_{\sigma}^{-1} X^{\prime} \\
{[\mathrm{MPa}]}\end{array}$ & $\begin{array}{l}\gamma_{\sigma}^{-1} X^{\prime} \\
{[\mathrm{MPa}]}\end{array}$ & $\begin{array}{l}\gamma_{\sigma}^{-1} S \\
{[\mathrm{MPa}]}\end{array}$ & $\begin{array}{l}\rho[\mathrm{kg} \\
\left.\mathrm{m}^{-3}\right]\end{array}$ \\
\hline Gelcoat & 3.44 & 0.3 & - & - & - & 1,235 \\
\hline Foam & 0.1425 & 0.3194 & 2.083 & 1.563 & 1.250 & 130 \\
\hline
\end{tabular}




\subsubsection{The Big Adaptive Rotor Structural Designs}

This section covers the BAR designs including airfoil polar data, geometry of the blades, and structure of the blade. We chose the Aeronautical Research Institute of Sweden (FFA) airfoils used in the IEA Wind Task 37 offshore reference wind turbine. Because the minimum thickness of the set was $21 \%$, we added the thinner airfoil NACA63-618 to the set. In addition, Sandia designed a $50 \%$ thick airfoil with a $10 \%$ thick flat-back trailing edge. The polars were computed running the panel solver XFOIL using default settings. Table 11 lists the airfoils used, whereas Figure 29 and Figure 30 show the airfoil coordinates and polars, respectively.

Table 11. Airfoils Used in the Design Process

\begin{tabular}{|l|c|}
\hline Airfoil & Relative Thickness (\%) \\
\hline Cylinder & 100 \\
\hline FFA-W3-360 & 36 \\
\hline FFA-W3-241 & 24.1 \\
\hline NACA63-618 & 18 \\
\hline SNL-FFA-W3-500 & 50 \\
\hline FFA-W3-301 & 30.1 \\
\hline FFA-W3-211 & 21.1 \\
\hline
\end{tabular}




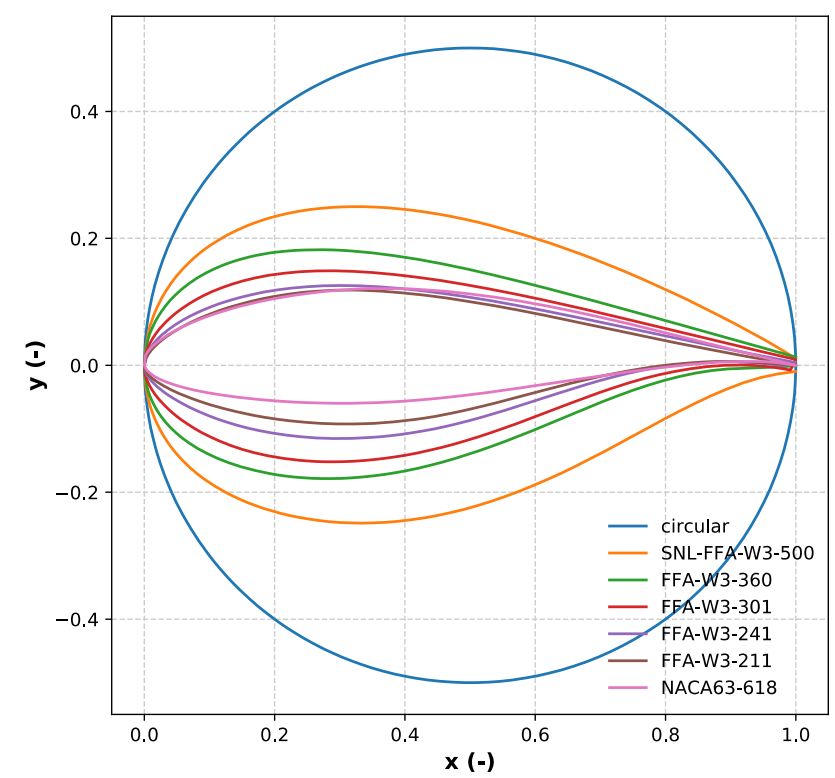

Figure 29. Coordinates of the airfoils used in the design of the BAR flexible blades
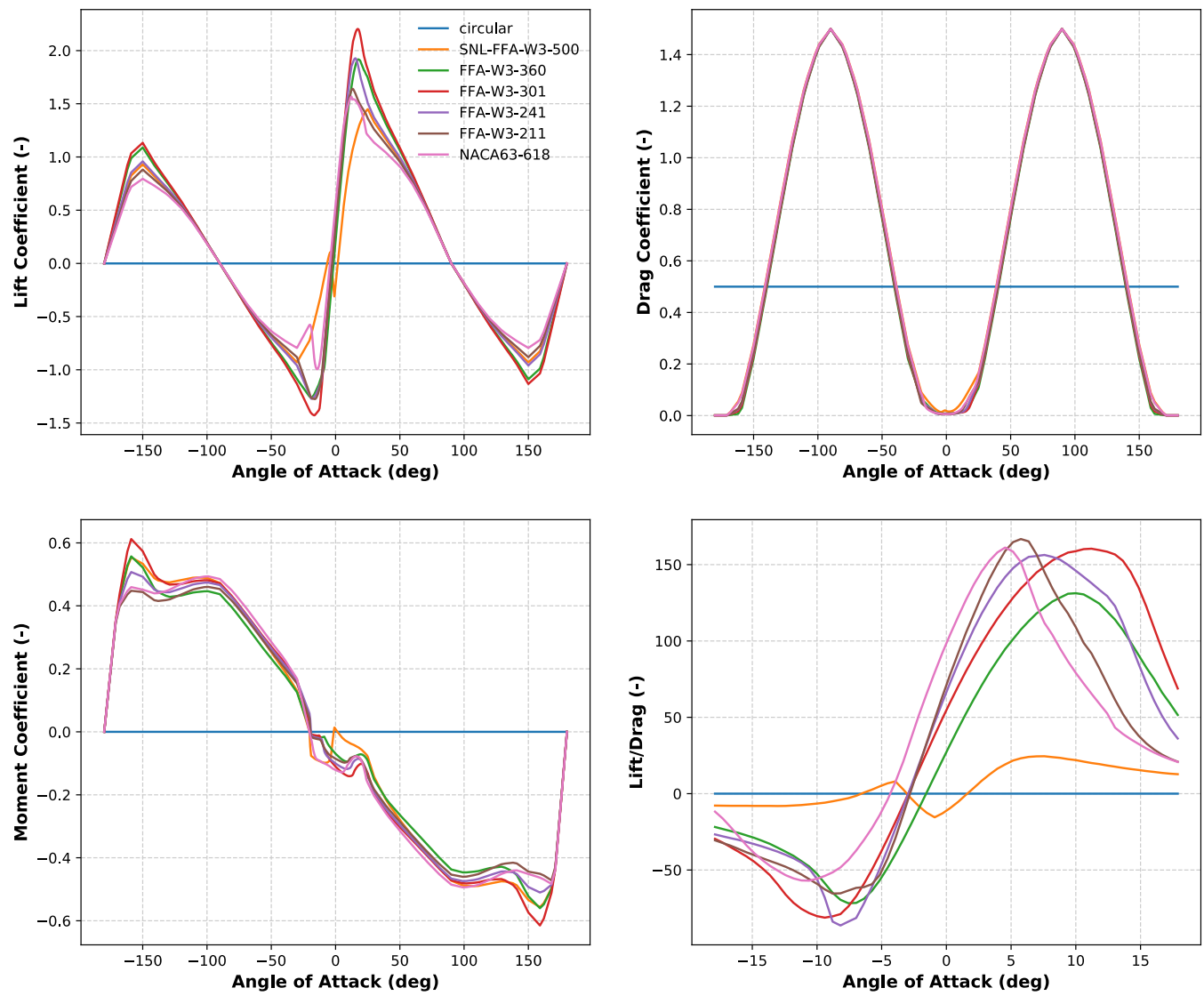

Figure 30. Polars of the airfoils used in the design of the BAR flexible blades

The wind turbine blade configurations are described in Figure 31 in terms of chord, twist, relative thickness, and prebend distributions. 

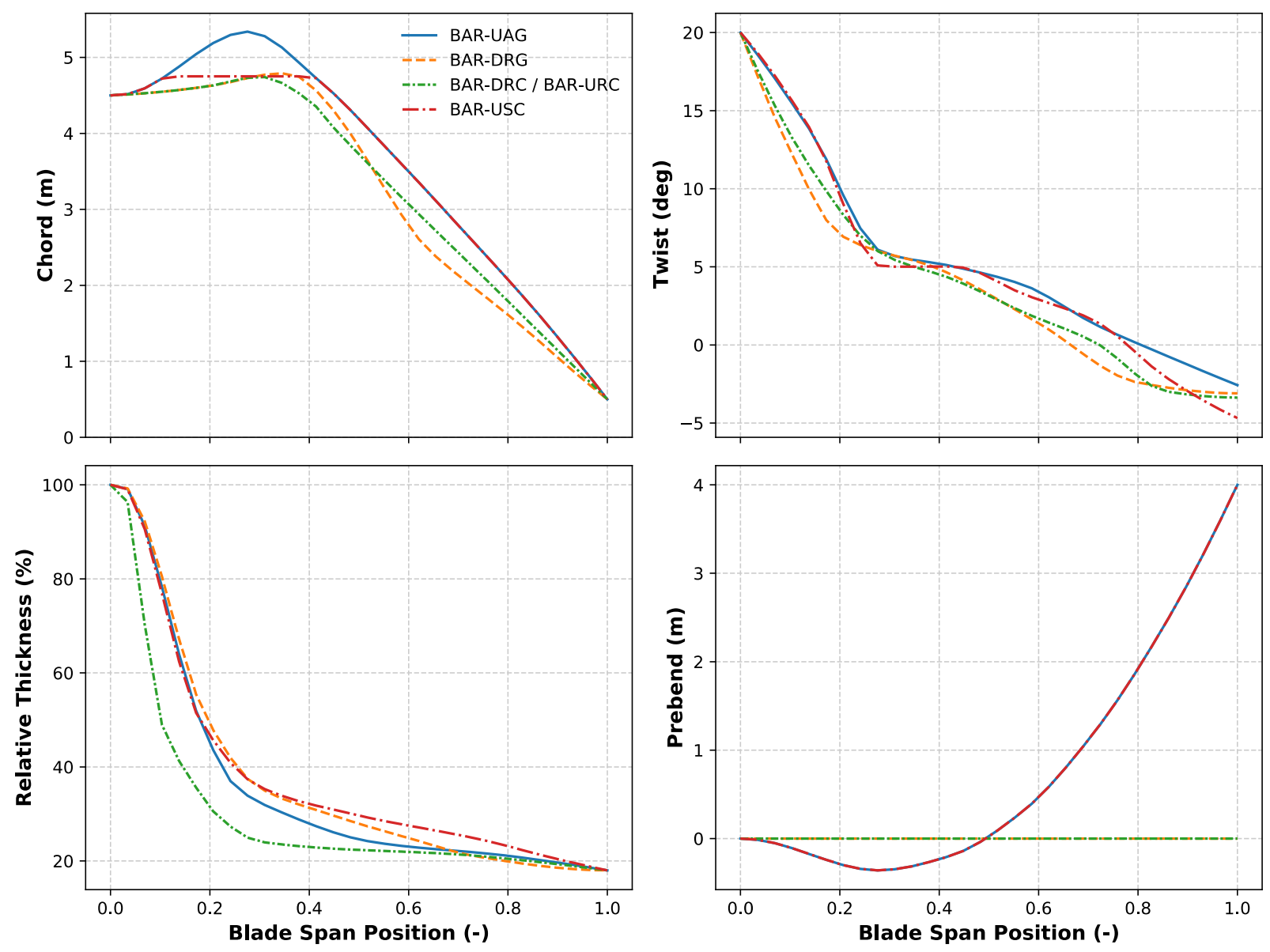

Figure 31. Chord, twist, relative thickness, and prebend distributions of the five BAR designs

Alleviating the limit on maximum tip deflection either by using a downwind turbine configuration or by increasing tilt angle allowed us to reduce the blade flapwise stiffness and mass in the design iterations conducted in both WISDEM and NuMAD. In NuMAD, the resulting flexible designs became primarily driven by the constraints on material rupture and buckling rather than tip deflection. Results also showed that rail-transportable blades are confined to a more limited range of feasible design space because they must strike a compromise between conflicting constraints on structural performance and flexibility. Figures 32 through 37 show the comparisons of the final optimized spanwise thickness distributions for each component in the different blade designs. 

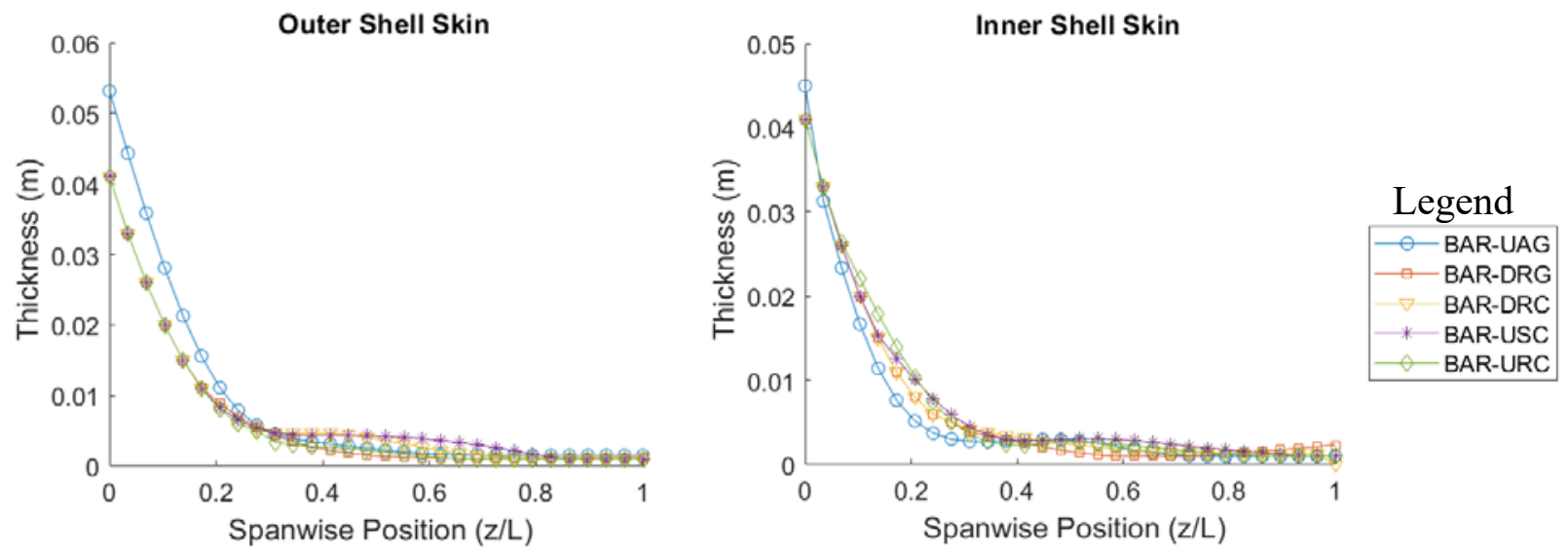

Figure 32. Spanwise thickness distribution of shell skins for optimized BAR designs
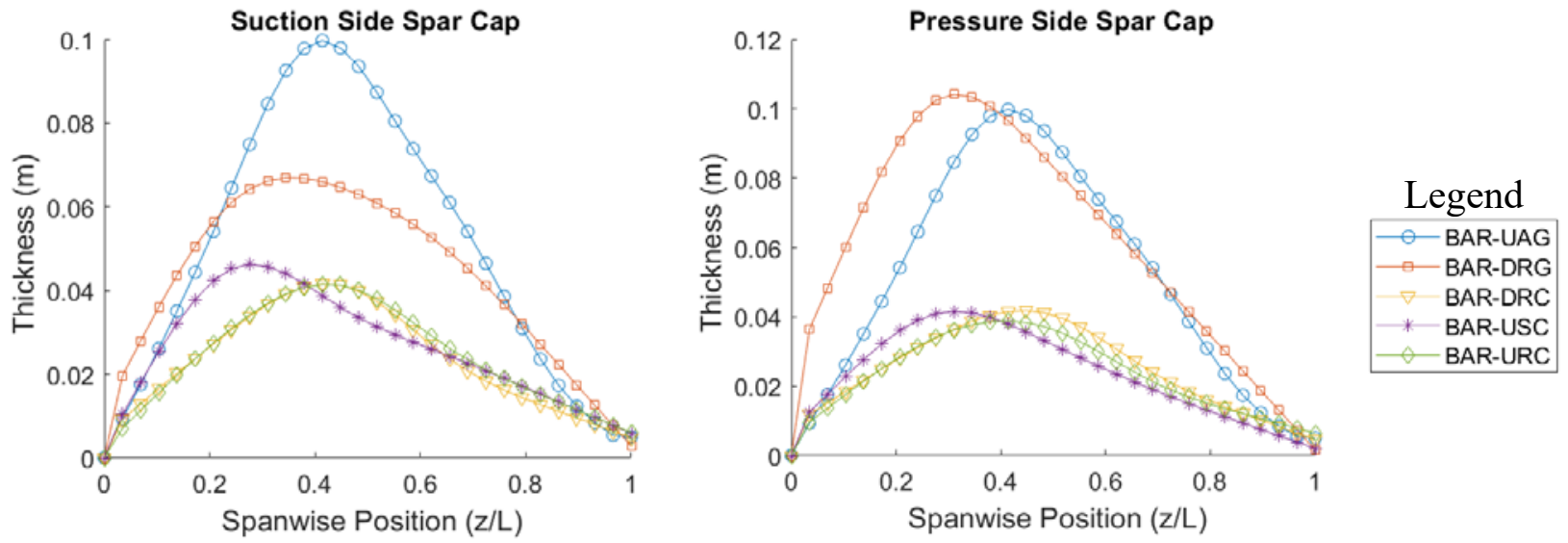

Figure 33. Spanwise thickness distribution of spar caps for optimized BAR designs
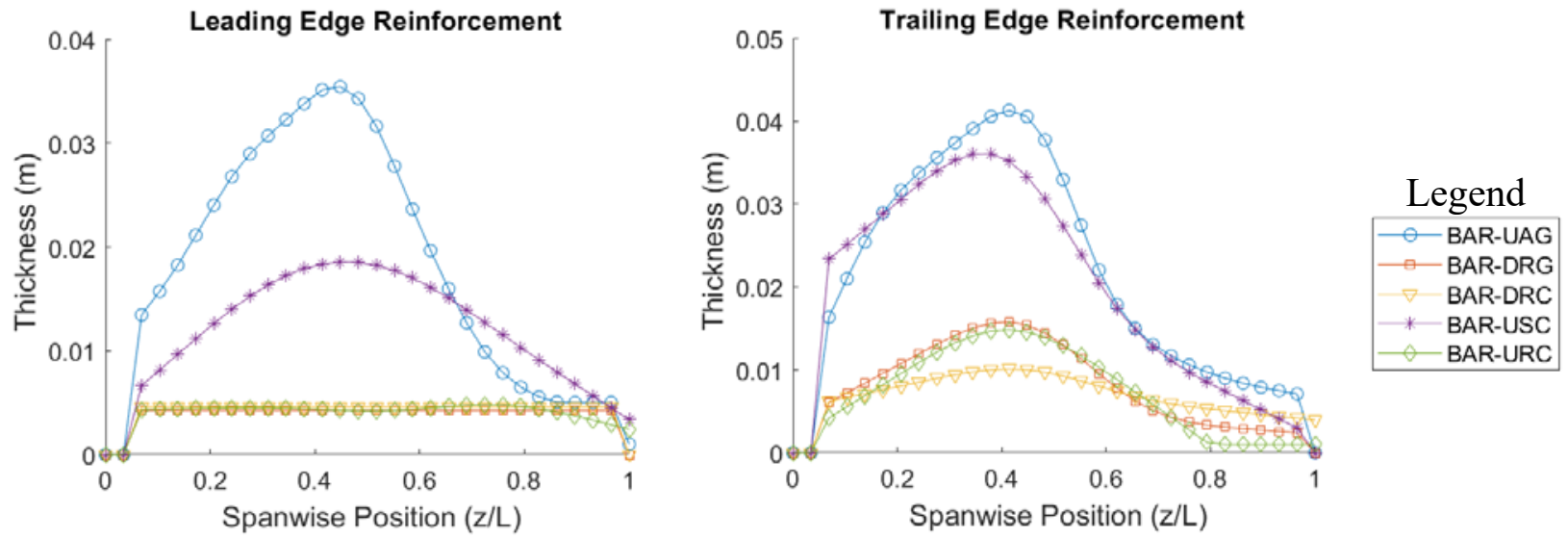

Figure 34. Spanwise thickness distribution of leading- and trailing-edge reinforcement for optimized BAR designs 

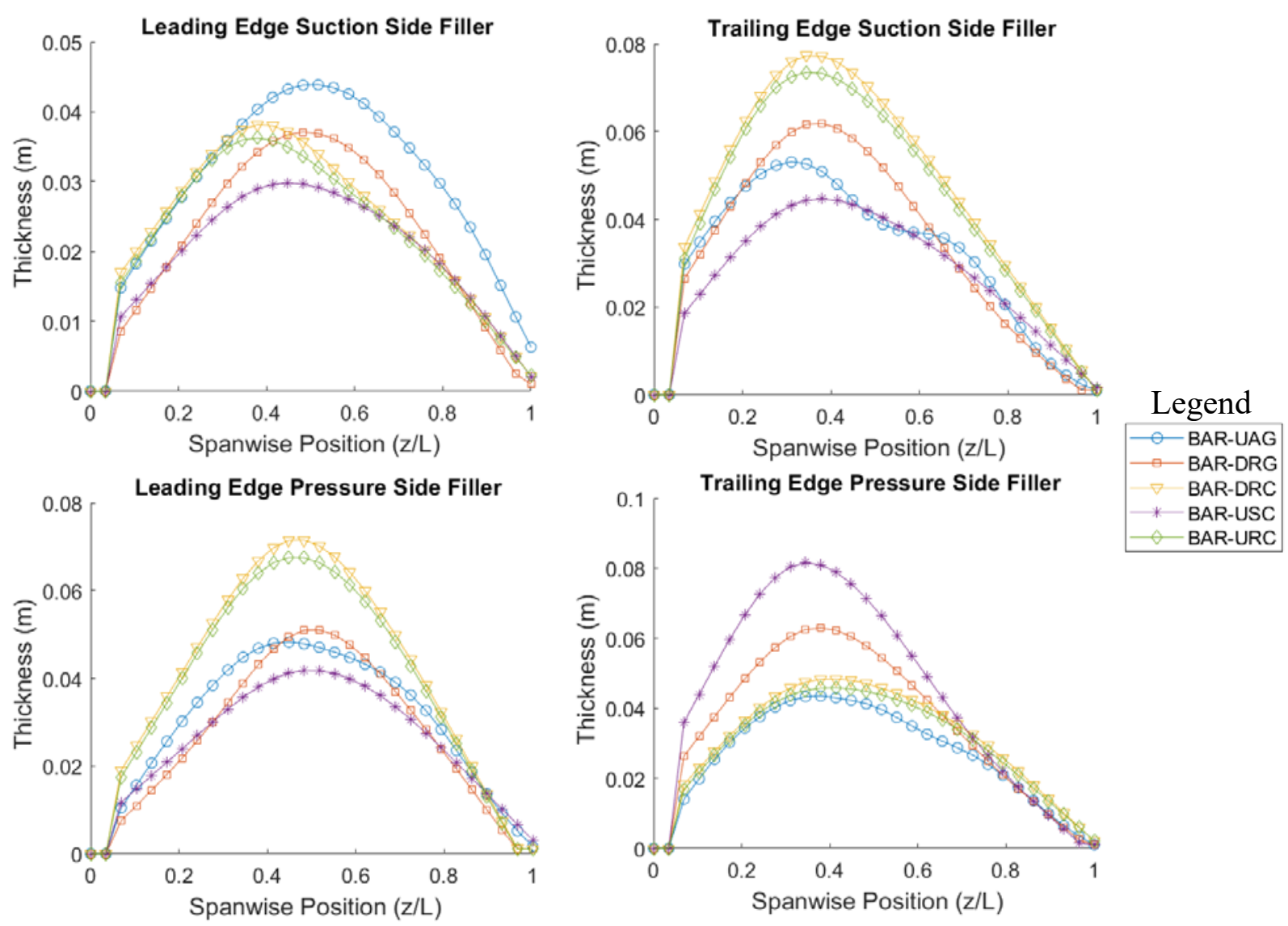

Figure 35. Spanwise thickness distribution of core filler for optimized BAR designs
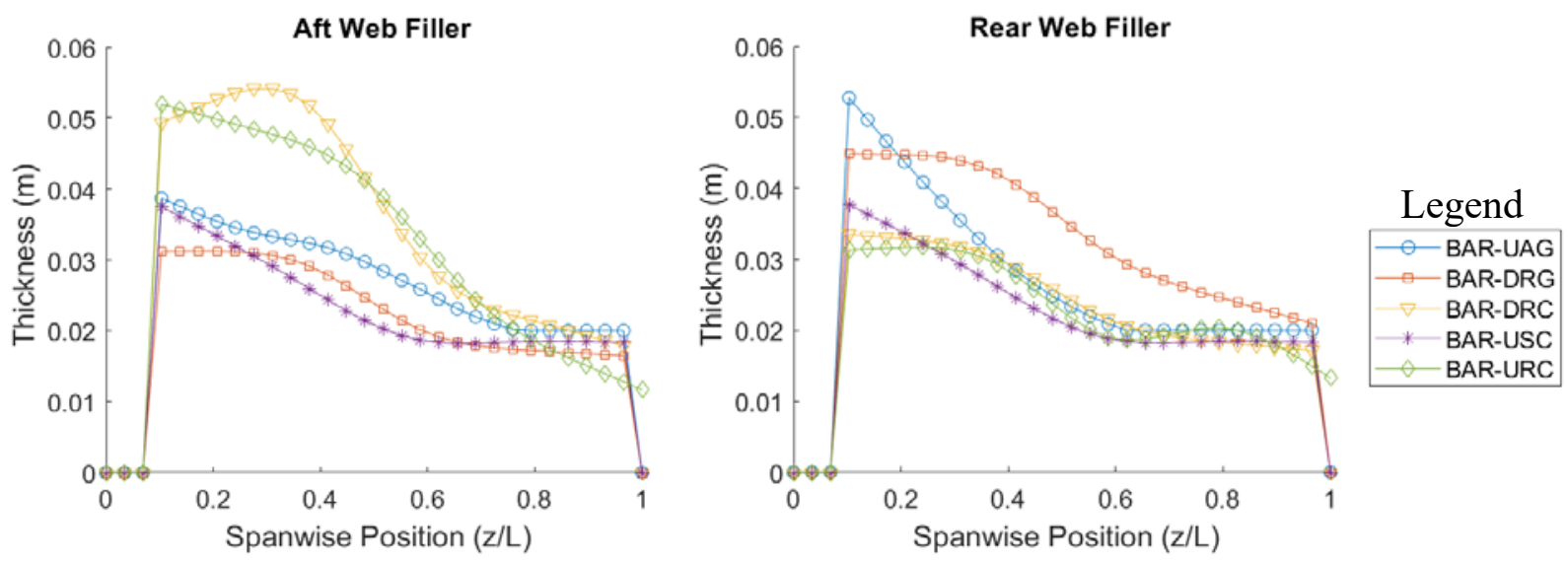

Figure 36. Spanwise thickness distribution of shear web filler for optimized BAR designs 

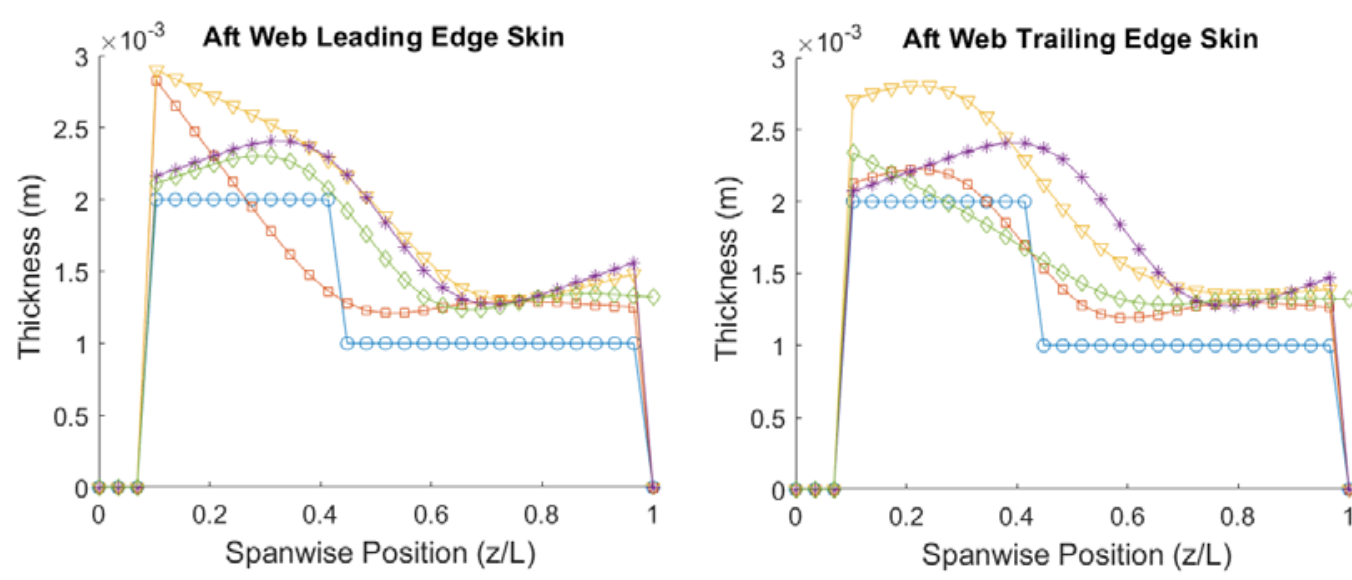

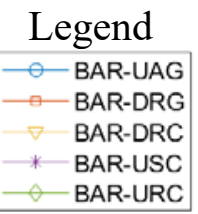
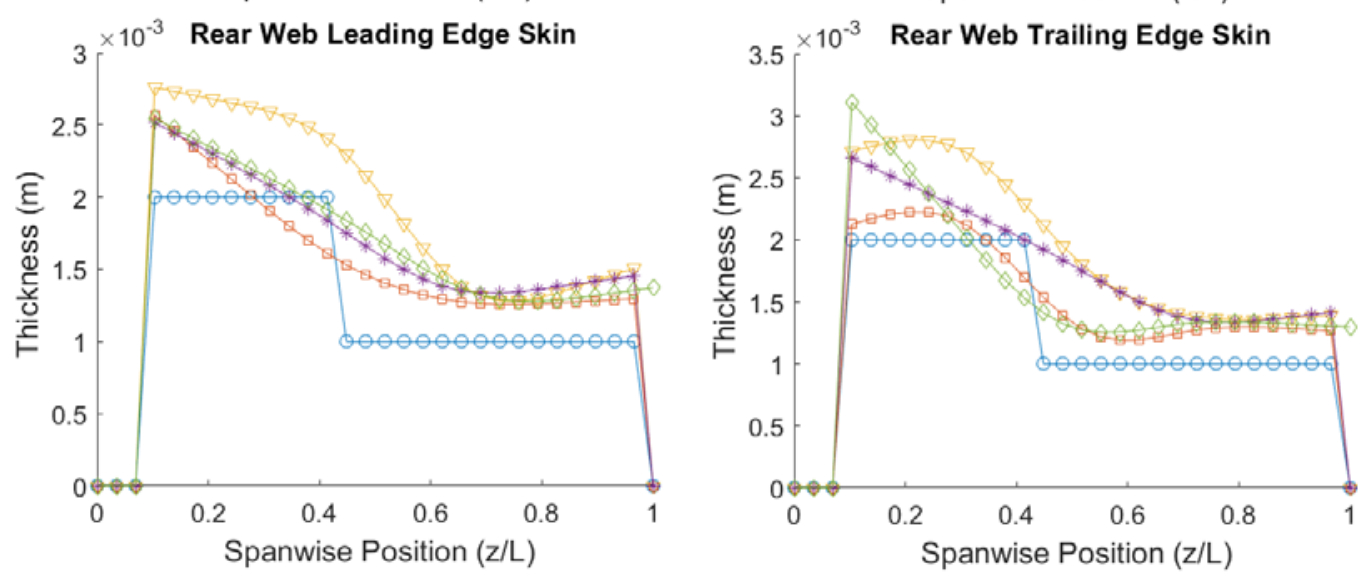

Figure 37. Spanwise thickness distribution of web skins for optimized BAR designs

The primary driving constraints in the final state for each blade design and the components and approximate spanwise location they pertain to, if applicable, are highlighted in Table 12. For designs that are primarily driven by material rupture and/or buckling - namely BAR-DRG, BAR-DRC, and BAR-URC - the rupture failure index is plotted along the blade structure in Figure 38, Figure 39, and Figure 40. The displacement plots for the most critical buckling modes for the same blades are shown in Figure 41, Figure 42, and Figure 43. These figures highlight key areas of potential vulnerability along the blade span, showing that discontinuous featuressuch as the ends of the shear webs and airfoil transitions - tend to be areas of relative weakness.

Table 12. Summary of Driving Constraints for Different Blade Designs in Structural Optimization

\begin{tabular}{|c|c|}
\hline Design & Driving Constraints \\
\hline BAR-UAG & Tip deflection \\
\hline BAR-DRG & $\begin{array}{l}\text { Rupture in shear web skins, } 20 \% \text { span } \\
\text { Buckling in leading-edge suction-side panels, } 25 \% \text { span }\end{array}$ \\
\hline BAR-DRC & $\begin{array}{l}\text { Rupture in shear web skins, } 10 \%-15 \% \text { span } \\
\text { Rupture in trailing-edge reinforcement, } 10 \% \text { span } \\
\text { Buckling in suction-side spar caps, } 25 \% \text { span }\end{array}$ \\
\hline BAR-USC & Tip deflection \\
\hline BAR-URC & $\begin{array}{l}\text { Rupture in shear web skins, } 10 \%-15 \% \text { span } \\
\text { Rupture in trailing-edge reinforcement, } 10 \% \text { span } \\
\text { Buckling in suction-side spar cap, } 25 \% \text { span }\end{array}$ \\
\hline
\end{tabular}


(a)

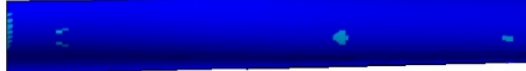

(b)

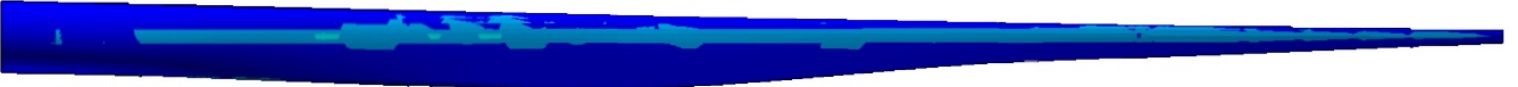

(c)

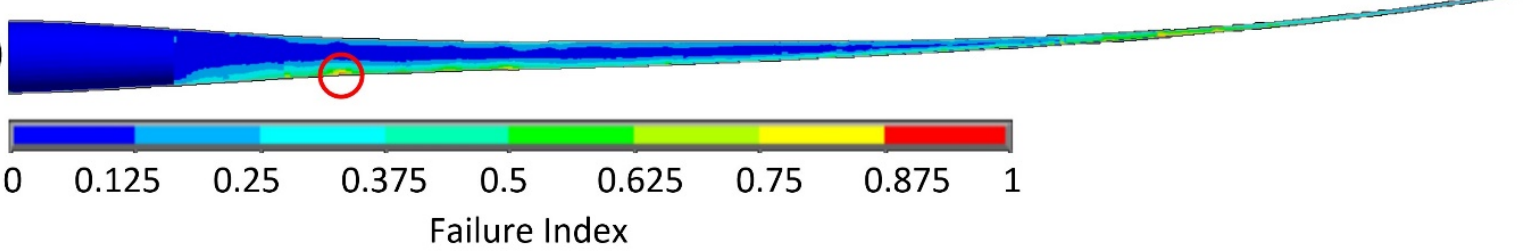

Figure 38. Tsai-Wu failure index under loading for BAR-DRG from the (a) suction side, (b) pressure side, and (c) trailing edge (cutaway view). The vulnerable point is circled in red.

(a)

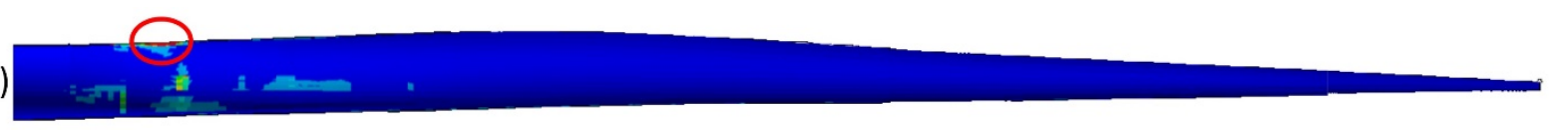

(b)
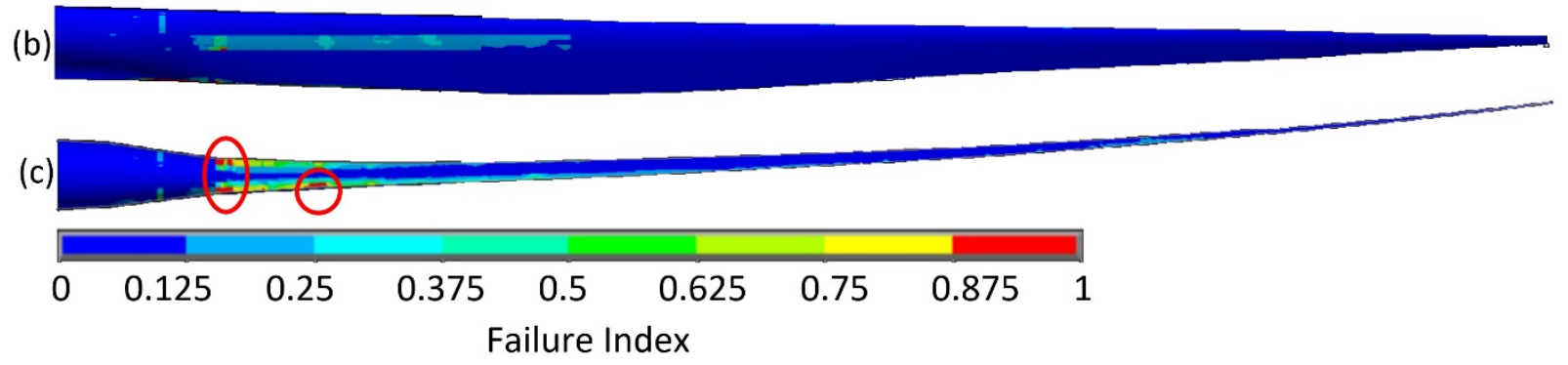

Figure 39. Tsai-Wu failure index under loading for BAR-DRC from the (a) suction side, (b) pressure side, and (c) trailing edge (cutaway view). Vulnerable points are circled in red. 
(a)

(b)

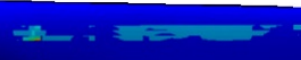

(c)

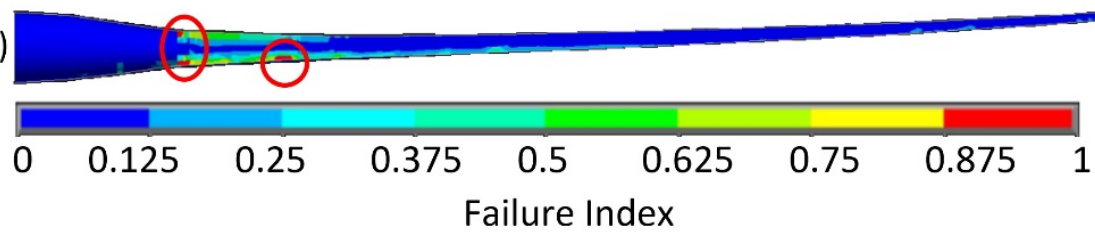

Figure 40. Tsai-Wu failure index under loading for BAR-URC from the (a) suction side, (b) pressure side, and (c) trailing edge (cutaway view). Vulnerable points are circled in red.

(a)

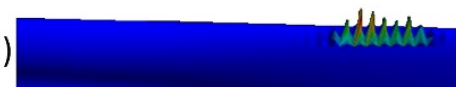

(b)

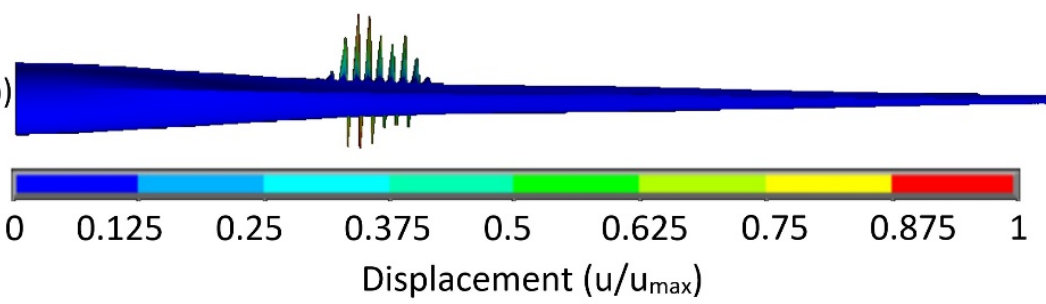

Figure 41. Displacement plot of most critical buckling mode under loading for BAR-DRG from the (a) suction side and (b) trailing edge

(a)

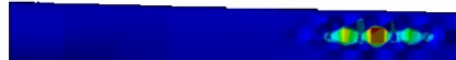

(b)

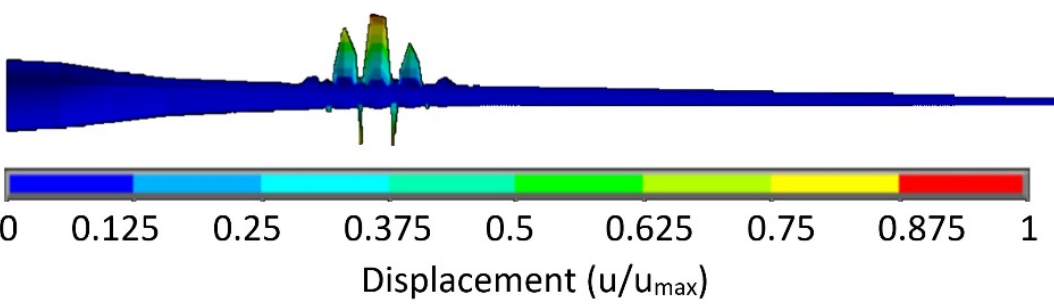

Figure 42. Displacement plot of most critical buckling mode under loading for BAR-DRC from the (a) suction side and (b) trailing edge 


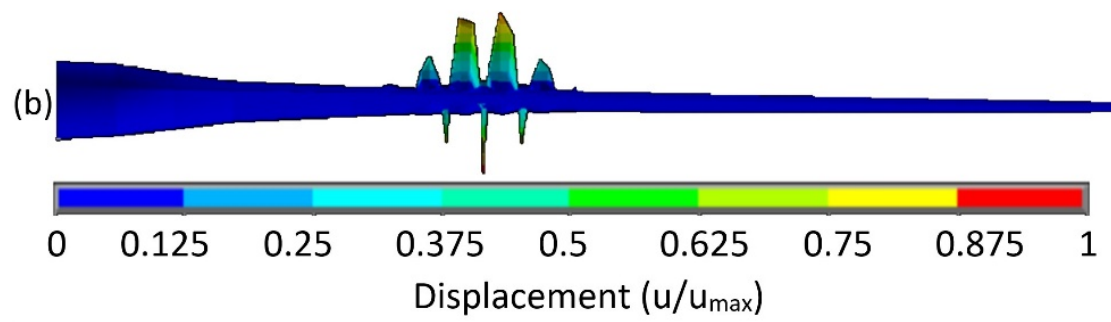

Figure 43. Displacement plot of most critical buckling mode under loading for BAR-URC from the (a) suction side and (b) trailing edge

Table 13 provides a summary of the five BAR designs. BAR-USC generates the highest aerodynamic performance at the expense of high loads and the highest turbine capital costs. On the contrary, BAR-DRC and BAR-URC show a different trade-off, wherein a lower AEP is more than balanced by the savings in capital costs. Overall, BAR-URC generates the lowest LCOE, $\$ 43.7 / \mathrm{MWh}^{-1}$, followed by BAR-DRC, with $\$ 44.4 / \mathrm{MWh}^{-1}$. The advantage of BAR-URC over BAR-DRC can be explained by the higher performance generated by the higher rotor area under loading that upwind rotors create compared to downwind ones. The highest LCOE is instead generated by the industry reference BAR-USC, which, despite the highest AEP, suffers from high costs coming from the blade mechanical joint and the high amount of carbon fiber in the spar caps needed to prevent tower strikes of the upwind rotor.

Table 13. Summary of the Design Characteristics of the BAR Flexible Rotors

\begin{tabular}{|c|c|c|c|c|c|}
\hline Design & BAR-UAG & BAR-DRG & BAR-DRC & BAR-USC & BAR-URC \\
\hline Orientation & Upwind & \multicolumn{2}{|c|}{ Downwind } & \multicolumn{2}{|c|}{ Upwind } \\
\hline Transport & Air & \multicolumn{2}{|c|}{ Rail } & Segmented & Rail \\
\hline Fabric spar caps & \multicolumn{2}{|c|}{ Glass } & \multicolumn{3}{|c|}{ Carbon } \\
\hline Prebend at tip (m) & 4 & \multicolumn{2}{|c|}{0} & 4 & 0 \\
\hline Cone/tilt angles (deg) & $4 / 6$ & $2 / 5$ & $2 / 5$ & $2 / 4$ & $4 / 8$ \\
\hline Cut-out speed $\left(\mathrm{m} \mathrm{s}^{-1}\right)$ & 25 & \multicolumn{2}{|c|}{19} & \multicolumn{2}{|c|}{25} \\
\hline Blade mass (tons) & 64.8 & 53.0 & 41.6 & 49.4 & 41.1 \\
\hline Blade cost (k\$) & 450 & 407 & 472 & 563 & 466 \\
\hline $\begin{array}{l}\text { Turbine capital costs (\$ } \\
\mathbf{k W}^{-1} \text { ) }\end{array}$ & 1375 & 1324 & 1343 & 1424 & 1336 \\
\hline AEP (gigawatt-hour) & 23.8 & 23.3 & 23.8 & 24.6 & 24.1 \\
\hline LCOE (\$ MWh-1) & 44.9 & 44.9 & 44.4 & 45.0 & 43.7 \\
\hline
\end{tabular}

The other conclusion that can be drawn from Table 13 is that the wind turbine blades adopting pultruded carbon fiber generate lighter blades, which, despite the higher blade costs, support higher power performance and lower systems costs, ultimately resulting in lower values of LCOE. In this scenario, we conducted a further investigation in NuMAD comparing the optimized designs with carbon composite spar caps (BAR-DRC, BAR-USC, and BAR-URC) using the industry-standard unidirectional composite and blades adopting heavy-tow unidirectional composite. The comparison indicated only slight differences in the optimized 
designs, but thanks to the relative low cost of the heavy-tow composite material, the results suggested a significant potential reduction in blade cost resulting from the use of heavy-tow carbon composite spar caps. Table 14 compares the overall blade cost analysis results for each case. Notably, because the design solutions were similar between industry-standard and heavytow carbon fiber, the design iterations for the blades with heavy-tow carbon did not loop back to WISDEM.

Table 14. Comparison of Total Cost Between Blades Adopting Industry-Standard Unidirectional Carbon Composite Spar Caps vs. Heavy-Tow Unidirectional Carbon Composite Spar Caps

\begin{tabular}{|c|c|c|c|}
\hline Design & $\begin{array}{c}\text { Total Cost, } \\
\text { Industry Standard }\end{array}$ & $\begin{array}{c}\text { Total Cost, } \\
\text { Heavy Tow }\end{array}$ & $\begin{array}{c}\text { Percentage } \\
\text { Reduction }\end{array}$ \\
\hline BAR-DRC & $\$ 472$ & $\$ 413$ & $12.6 \%$ \\
\hline BAR-USC & $\$ 563$ & $\$ 513$ & $8.9 \%$ \\
\hline BAR-URC & $\$ 466$ & $\$ 416$ & $10.6 \%$ \\
\hline
\end{tabular}

In the next phase of the BAR project, the team plans to increase the fidelity of the cost modeling to include the impacts of transportation costs. AEP will be calculated using OLAF and BeamDyn to determine if there are any inaccuracies from the BEM model that might skew the results. Finally, advanced control methods will be implemented for the downwind concept to increase cut-out wind speed and reduce loads for the shutdown cases.

\subsubsection{Inflatable Blade}

The goal of the inflatable blade concept was to enable larger rotors by mitigating the transportation constraints in two ways: (1) by reducing the maximum chord and associated stiffness during transportation (coupled with on-site manufacturing), and (2) by reducing the blade weight. This is accomplished by decoupling the external aerodynamic shape from the internal load-carrying structure, then inflating the external shape on-site. The research objective was to design a wind turbine blade that matched the structural stiffness of a conventional blade but with reduced mass and cost. Matching the stiffnesses reduces many potential risks associated with inflatable blades, such as shape deformation and unsteady aerodynamics. This research studied the effects of different structural architectures, materials and composites, fabrication techniques, and topologies on the structural stiffnesses, mass, and cost of the blade. Inflatable blade designs were compared to the BAR-UAG baseline blade, which had been matured through several structural iterations. Results showed that the inflatable blade was able to successfully match the flap, edge, and torsional stiffness of the baseline blade while reducing the mass by $15 \%-55 \%$ and the blade material cost by $5 \%-15 \%$ along the blade span. This was a result of using different materials and architectures for the structure of the beam.

The team made multiple design decisions to reduce the risk and increase the technology level of this inflatable blade concept to the greatest extent possible. We employed conventional materials and composite manufacturing techniques in combination with incorporating established textile manufacturing processes from other industries. On-site manufacturing offers several opportunities over traditional factory manufacturing, and we explored solutions to the technical challenges of on-site manufacturing. On-site manufacturing introduced high uncertainty in the costs. Loads analysis, loads transfer, and higher-fidelity structural analysis (fatigue, buckling, and so on) are open questions for follow-on work. This research demonstrated that this inflatable 
blade concept is feasible and practical and can offer some significant blade mass and material cost reductions. More details can be found in (Mendoza et al. 2021).

\subsubsection{Bi-Wing Blade}

The bi-wing blade design concept uses two inboard blade sections that join together outboard. This allows for a lower chord length inboard as well as the potential to remove the nonloadbearing structure (i.e., panels and aerodynamic shells) from the structure, ideally leaving only airfoil-shaped columns. Previous investigations (Roth-Johnson, Wirz, and Lin 2014) found a potential for a $46 \%$ reduction in mass and a $25 \%-35 \%$ reduction in tip deflection compared to conventional designs. Further, (Ragheb and Selig 2011) found the possibility of $\mathrm{Cl} / \mathrm{Cd}$ increases of $40 \%-60 \%$ when multiple airfoil sections were used, depending on the arrangement. The challenges to this design are potential increased drag from structural interference between the airfoils; controlling beam buckling in the downwind, inboard section; and the requirement of a blade joint. Given the aforementioned issues with pitch rates of large flexible blades, however, and given that previous studies constrained the blade to having a joined, conventional, round root section, the team investigated a modified concept. The design allows the inner blade sections to be separated farther from each other than the root diameter to create higher structural efficiency in the flap direction. Additionally, the root sections are assumed to be fixed, with the outboard joint acting as the pitch mechanism. Previous studies by Agarwala and Ro (2015) found partial span pitch control of the outer $30 \%$ of a blade to be sufficient for load control; thus, the concept might be able to solve both the logistics and control challenges that have been identified by the project as well as potentially decrease blade mass.

For this study, we used a Prandtl/Glauert empirical interference lift reduction for the aerodynamics of the inboard section. Increased drag from the joint is not accounted for, but neither is decreased drag from thinner airfoils that can potentially be used inboard. A simplified structure was used, assuming a rigid joint connecting bi-plane sections to the outboard section. We did not account for panel buckling because it is typically addressed with lightweight core materials. We assumed a simplified structural model of the inboard sections, whereby the airfoil sections are assumed to be mostly constructed of solid unidirectional glass composite. Inboard stiffnesses were targeted to be at least matched, whereas we assumed the outboard section to be identical to the reference design blade. Finally, we calculated the pitch system mass according to the NREL Wind Turbine Design Cost and Scaling Model (Fingersh, Hand, and Laxon 2006). Conclusions from this investigation are as follows:

- The glass design has $15 \%$ reduced mass and cost compared to the BAR-UAG baseline blade.

- The calculated pitch system mass is approximately $15 \%$ of the estimated joint mass in the Supersized Wind Turbine Blade study by DNV GL.

- There are increases in edgewise bending moment from the pitch system but reasonable strains from offset elements.

- Aerodynamic penalties are small for given chord/separation distances.

- Beam buckling is not trivial to eliminate, but it can be done.

- A larger downwind cross section (approximately $65 \%$ of the reference chord) and a smaller upwind cross section (approximately $35 \%$ of the reference chord) increases buckling resistance. 
- Centrifugal effects increase buckling resistance, but they are small.

- Edgewise stiffness can be recovered only through offsetting sections chordwise or with the addition of a third segment.

- Chordwise offsets will cause bend-twist coupling to some extent, so direction can be chosen to pitch toward feather to alleviate loads.

\subsubsection{Five-Bladed Rotors}

The team conducted a preliminary investigation into the potential of wind turbine rotors with more than three blades because to make the blade transportable by rail, it needs to be more flexible, and a rotor with more blades will have smaller chords and inherently greater flexibility. We evaluated a five-bladed rotor in WISDEM starting from the baseline design BAR-UAG. The tip-speed ratio was maintained at its nominal value of 10.5 , whereas the chord distribution was reduced by $3 / 5$. In terms of blade internal structure, we did not change the thickness of all components, the width of the spar caps, the width of the leading- and trailing-edge reinforcements, and the shear web positions, whereas we scaled the width of the sandwich panels following the smaller chord. By adopting these simple changes, each blade became $26 \%$ lighter and $33 \%$ cheaper than the baseline, but the rotor became $18 \%$ heavier and $12 \%$ more expensive. In addition, the reduced absolute thickness of the blades generated by the smaller chord resulted in lower flapwise stiffness, and the blades violated the tip deflection constraint by $93 \%$. The blade also showed dangerous crossings in the Campbell diagram, with the first flapwise frequency between the first and second rotor harmonics, and the first edgewise frequency between the second and third harmonics. The tower was not redesigned, but the rotor nacelle assembly increased by $12 \%$, and the maximum rotor thrust stayed constant. AEP increased only marginally $(+0.4 \%)$. Satisfying the blade-tower clearance constraint would have caused a tremendous increase in the spar-cap thickness and, in turn, the blade mass.

Therefore, it was decided to stop investigating the five-bladed rotor concept. Further research could continue the studies by turning the rotor downwind, by redesigning the chord more finely than the crude scaling adopted here, and/or by varying the tip-speed ratio. Still, we did not believe that increasing the number of blades could successfully address the challenges of modern wind turbine rotors.

\subsubsection{Distributed Aerodynamic Controls}

NREL developed capability for codesign of a blade with the DAC using WISDEM and OpenFAST through this project. The publication "Distributed Aerodynamic Control Using Active Trailing-Edge Flaps for Large Wind Turbines" (Feil et al. 2020a) was released, and the outcomes are summarized here:

Recent efforts for developing an advanced multidisciplinary modeling environment that combines the capabilities of multiple tools, including OpenFAST, WISDEM, ROSCO, and XFoil, are presented. The approach is well suited to analyze and optimize arbitrary DAC devices and enables comprehensive codesign studies. This work focuses on presenting the newly developed modeling capabilities and evaluates different trailing-edge-flap designs and locations along the radius of the modified BAR baseline blade. The following specific conclusions have been drawn: 
- Within a numerical optimization framework, introducing automated controller tuning methods for a specific blade and DAC design was essential to evaluate performance characteristics of DAC devices. To enable DAC optimization, the current workflow is embedded in WISDEM and automatically tunes the pitch and trailing-edge-flap controller.

- The conducted parameterization and optimization studies showed that a specific flap design's ability to achieve a desired control objective peaked at the radial station of maximum thrust per unit span.

- The area of optimal flap placement along the blade span was relatively flat and therefore not sensitive to small placement variations within the outboard $40 \%$ of the blade.

- In general, larger flaps had a greater influence on the control objective. The characteristics between different sizes were nonlinear and depended on the load distribution along the blade span (i.e., the amount of thrust that is being manipulated).

- DAC devices were found effective in mitigating loads during both coherent gusts and turbulent wind. This helped mitigate the detrimental effects of slow blade-pitch rates that affect modern large rotors.

- Incorporating DAC devices can be used to improve system-wide performance metrics. The reduction of extreme and fatigue loads on the blade itself, as well as for associated components, such as the pitch-control system, has a large potential to help lower LCOE.

In conclusion, the established modeling environment is well-suited to investigate DAC devices for wind turbine application. Future work includes further improving the modeling capabilities by accounting for different DAC devices, effects on the blade-structural dynamics when integrating trailing-edge flaps, and optimizing the controller tuning parameters.

By developing cost functions, including reliability implications, to address the increased complexity and varying blade structure with integrated DAC devices, conducting codesign optimization studies over a range of design load cases, the established modeling environment will furthermore be applied to analyze the impact on the blade's design when using optimized DAC devices and to evaluate its potential for lowering LCOE. The current work on the design and controllability of DAC devices intends to further the understanding of such technologies, and therefore will support mitigating the challenges of current and future wind turbines that incorporate increasing blade length and flexibility.

\subsection{Subtask 4.D: Identification of Key Validation Needs for Phase II}

After starting with a large number of potential design innovations to enable the design, manufacture, transportation, and operation of low-specific-power rotors, Phase I of the BAR project down-selected to specific configurations and technologies that allow for the design of slender flexible blades. In the design and optimization process, the team identified several validation needs that, if not addressed, would be expected to lead to high uncertainty in the commercial design of these technologies. These needs are divided into three categories, as shown in Figure 44: highly flexible blades, distributed aero controls, and downwind rotors. 


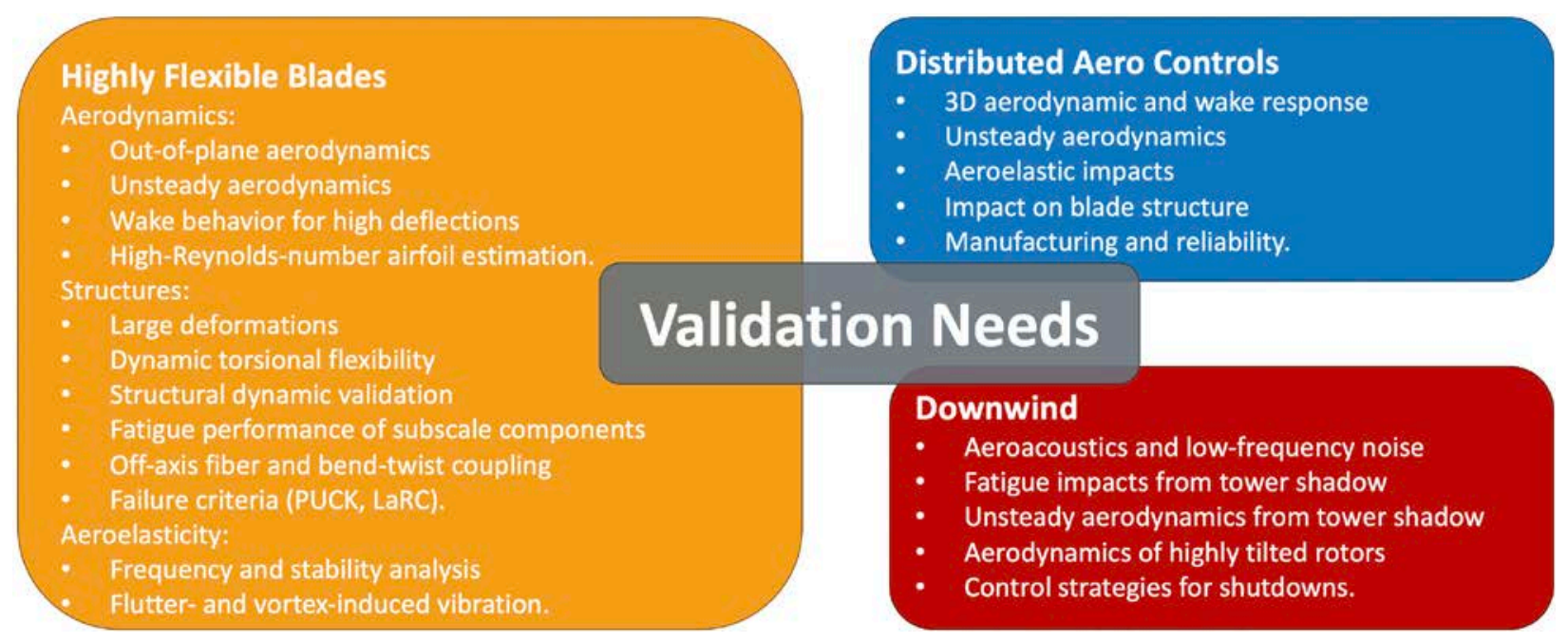

Figure 44. Validation needs for BAR Phase II

The identified validation needs for the BAR concepts include a comprehensive list of phenomena that, if validated, would remove many existing barriers to industry adoption of the technologies and, in turn, reduce the cost of land-based wind. For the highly flexible, controlled bending blade, there are many questions about the capability of the design tools to accurately predict the aerodynamics, structural impacts, stability, and coupled aeroelastic impacts of such a design. For the DAC concept, questions focus on the ability to model the aerodynamic and structural impacts of the device on the blades. For the downwind concept, there are questions about the ability of the models to accurately predict the impact of the tower wake on the noise emissions and the induced unsteady effects on the aerodynamics and structural dynamics of the blades. The comprehensive validation needs identified here have impacts beyond the BAR project and are in line with needs from other DOE projects, including high-fidelity modeling and the integrated systems design and analysis projects.

An ideal experimental validation campaign would include all these quantities of interest on a wind turbine at a scale similar to the BAR baseline turbine; however, such an experiment would easily cost tens of millions of dollars, if not more, and would likely require partnership with an existing turbine manufacturer. Alternatively, progress toward validating these technologies and phenomena can be made through targeted experiments at a variety of scales using existing DOE experimental infrastructure.

In the next phase of the BAR project, we propose four groups of experiments to address the validation needs: (1) downwind experiments, (2) materials and substructures experiments, (3) stability and flutter experiments, and (4) DAC experiments. Figure 45 shows the phenomena from the validation needs on the left. The hierarchy identifies which phenomena can be resolved at a given scale. Further, the figure shows the validation hierarchy in terms of the proposed Phase II experiments, and it shows which phenomena will be targeted with the experiments. Not all the validation needs are addressed in the proposed Phase II experiments. 
- Aeroacoustics/LFN

- Tower shadow effects

- Out-of-plane aerodynamics

- Unsteady aerodynamics

- Wake behavior for high deflections

- Fully coupled structural frequency

- Fully coupled flutter and stability

- Pitching/torsional flexibility

- High-Reynolds-number airfoil (2D) and rotor data (3D).

- DAC operation and aeroelastic response

- Large flapwise deformations of composite blades

- Flutter and stability of scaled blades

- Structural frequency of scaled blades

- Out-of-plane aerodynamics

- Dynamic torsional flexibility

- Unsteady aerodynamics of scaled blades.

- Mechanical properties of composites

Bend-twist coupling of composites

- Fatigue performance of subscale components

- High-Reynolds-number airfoil estimation.

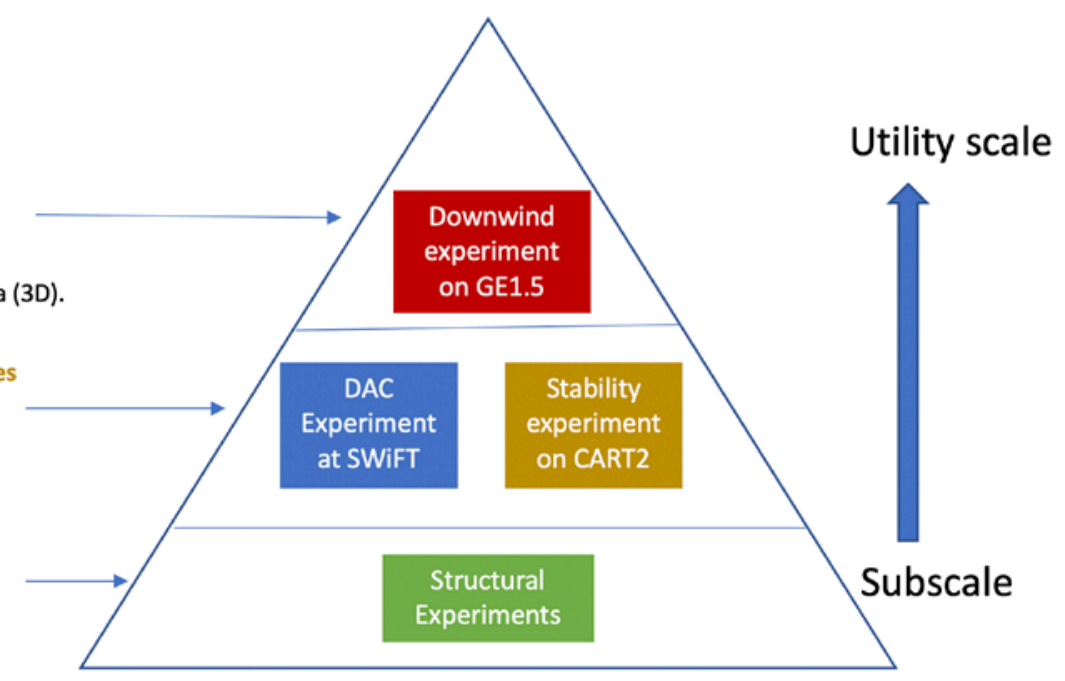

Figure 45. Resolved phenomena at scale

Note: SWiFT (Scaled Wind Farm Technology) facility; CART2 (two-bladed Controls Advanced Research Turbine)

The proposed Phase II experiments were partly selected based on equipment availability and importance ranking of the validation needs. All the experimental facilities are already owned by DOE and operated by DOE labs. The downwind experiment is proposed at utility scale on a GE 1.5-MW wind turbine located at NREL. At the intermediate scale, the DAC and stability experiments are proposed on the Sandia SWiFT turbines and NREL CART2 turbine, respectively. The SWiFT turbines are Vestas V27 turbines with a rated power of $200 \mathrm{~kW}$, and the CART2 is a $600-\mathrm{kW}$ two-bladed downwind turbine with a rotor diameter of $43.3 \mathrm{~m}$. Finally, structural testing is proposed at subscale at NREL and Montana State University.

Some phenomena, such as flutter and stability, can be resolved at midscale, but they might still be missing some physics. For example, a wind turbine blade can easily be scaled in flapwise stiffness but not necessarily in edgewise stiffness, and although this is still useful for model validation, it would not be completely representative of a full-scale specimen especially regarding atmospheric conditions, Reynolds number, and gravity loads. Note that as the scale increases, the costs of the experiments also increase. These costs can quickly become prohibitive. In general, both the presence of the phenomena of interest and the measurement uncertainty increases with scale. The most relevant experiments, from an industry-adoption perspective, are most likely at full scale; however, there is value in the hierarchical approach to model validation by targeting specific phenomena at relevant scales.

Again, however, to fully address all the validation needs, the most ideal experiment for model validation and demonstration would be based on a multimegawatt wind turbine in a downwind configuration with highly flexible blades. To keep everything open source, this turbine would need to be designed and built by the experimental team in partnership with a turbine manufacturer. This would be a world-class experiment that would include detailed inflow and outflow characterization across the rotor plane and detailed structural and aerodynamic measurements. This experiment would be impactful beyond the BAR project and would provide 
useful validation data for high-performance computing models, wake models, and wind power plant models. A full-scale experiment is important because the Reynolds number does not scale in the field, whereas parameters such as blade stiffness scale only partially. Full-scale experiments would remove a large source of uncertainty and would allow for easier adoption by industry. The resources required for a full-scale demonstration and validation of technology are far greater than what is available for BAR Phase II. Such an experiment should be considered in the future if sufficient funding is allocated. 


\section{Task 5: Optimized Carbon Fiber for Wind Turbines}

\subsection{Subtask 5.A: Blade and Material Cost Model Development}

This task is a continuation of a previous project that was established to assess the commercial viability of developing cost-competitive, carbon-fiber composites specifically suited for the unique loading experienced by wind turbine blades. This project characterized carbon-fiber materials made using low-cost acrylic precursors derived from the textile industry in addition to a baseline carbon-fiber material common to the wind industry. The textile carbon-fiber materials are processed in a heavy-tow size, in which there are a large number of fibers in a tow bundle, with a resulting 3-4 times greater linear density. The cost modeling results revealed a 56\% reduction in cost for the study heavy-tow textile carbon-fiber material compared to the industrybaseline carbon fiber, with reductions in all production cost categories of raw materials, capital, labor, and energy. To assess the value of the novel textile carbon fiber, we preformed mechanical testing in the project to compare the two carbon systems in relevant material forms. For blade spar caps using carbon fiber, pultruded planks are produced as an intermediate step to blade manufacturing. Being an automated process, pultrusions ensure adequate and consistent wetting of the resin system on the fibers and on the process tensions and straightens the fibers in manufacturing, resulting in high-performing carbon-fiber systems with reduced variability. The pultrusion process employs carbon fiber in tow formats that are lower in cost than fabrics or preforms used for resin infusion processes. A pultrusion cost model has also been developed in the work to estimate the cost of this additional processing step to produce the intermediate composite form.

We used the cost modeling and mechanical testing results to estimate the model input performance and cost properties of the two carbon systems in a $68 \%$ fiber volume fraction pultrusion. In addition to the cost reduction for the heavy-tow textile carbon fiber, we estimated the material to have improvements in cost-specific properties for the pultruded composite compared to the baseline material. The heavy-tow textile carbon pultrusion was found to have a $22 \%$ increase in cost-specific tensile strength, a 56\% increase in cost-specific compressive strength, and a 100\% increase in cost-specific modulus (with an equivalent modulus for both systems). For wind turbines, the most important static properties are the compressive strength and modulus, as tensile strength is typically underused because of spar-cap symmetry with similar magnitude tensile and compressive loads from bending. Additionally, the fatigue properties appear to be more stable with the heavy-tow textile fiber than for the baseline materials. The absolute strength for the heavy-tow textile carbon pultrusion was less than the baseline, so it is also important to compare the performance in reference blade models. We performed this blade design comparison for a high-energy-capture, 3-MW, land-based design as well as for a high-wind-resource, 10-MW, offshore reference design. In both cases, the heavytow textile carbon fiber was found to reduce the spar-cap material cost in the blade by approximately $40 \%$ over the baseline carbon, producing blades with a similar weight that were approximately 25\% lighter than a fiberglass spar cap design (with the higher-strength baseline carbon blade being slightly lighter). The final results can be found in (Ennis et al. 2019).

This task is a continuation of the prior work and extends the analysis beyond the blade spar cap and by assessing a broader range of carbon-fiber materials. The task work is ongoing, and results 
will be published in a separate final report (Ennis et al. Forthcoming). The task work in this project is divided into two main subtasks, as described in the following two sections.

\subsection{Subtask 5.A: Blade and Material Cost Model Development}

This subtask includes characterization work that will be used to generate inputs for blade design studies. A major deliverable is producing a catalog of carbon-fiber cost and property modeling and data from this task and related work to associate carbon-fiber cost and mechanical properties for the most relevant materials and forms to the wind turbine blade application. Figure 46 shows the tow properties of the 10 carbon-fiber materials that constitute the carbon-fiber catalog, which span various precursor materials and processing approaches. Component-level testing is being performed to move up in the testing hierarchy (from coupon-level testing in the original project) to improve confidence in mechanical performance in more relevant structures. The two main objectives of the mechanical testing are to produce data that illustrate (1) the variation in mechanical performance when testing in relevant structures and (2) the impact of realistic combined loading in fatigue performance (compared to pure axial loading and intermittently applied multiaxial loads). We are further refining the pultrusion cost model to provide more accurate manufacturing cost predictions with varying dimension and processing speeds consistent with carbon- and glass-fiber pultrusions. This model will be used to compare various approaches in designing with carbon fiber as well as to assess the value of glass pultrusions for wind turbine spar caps. 


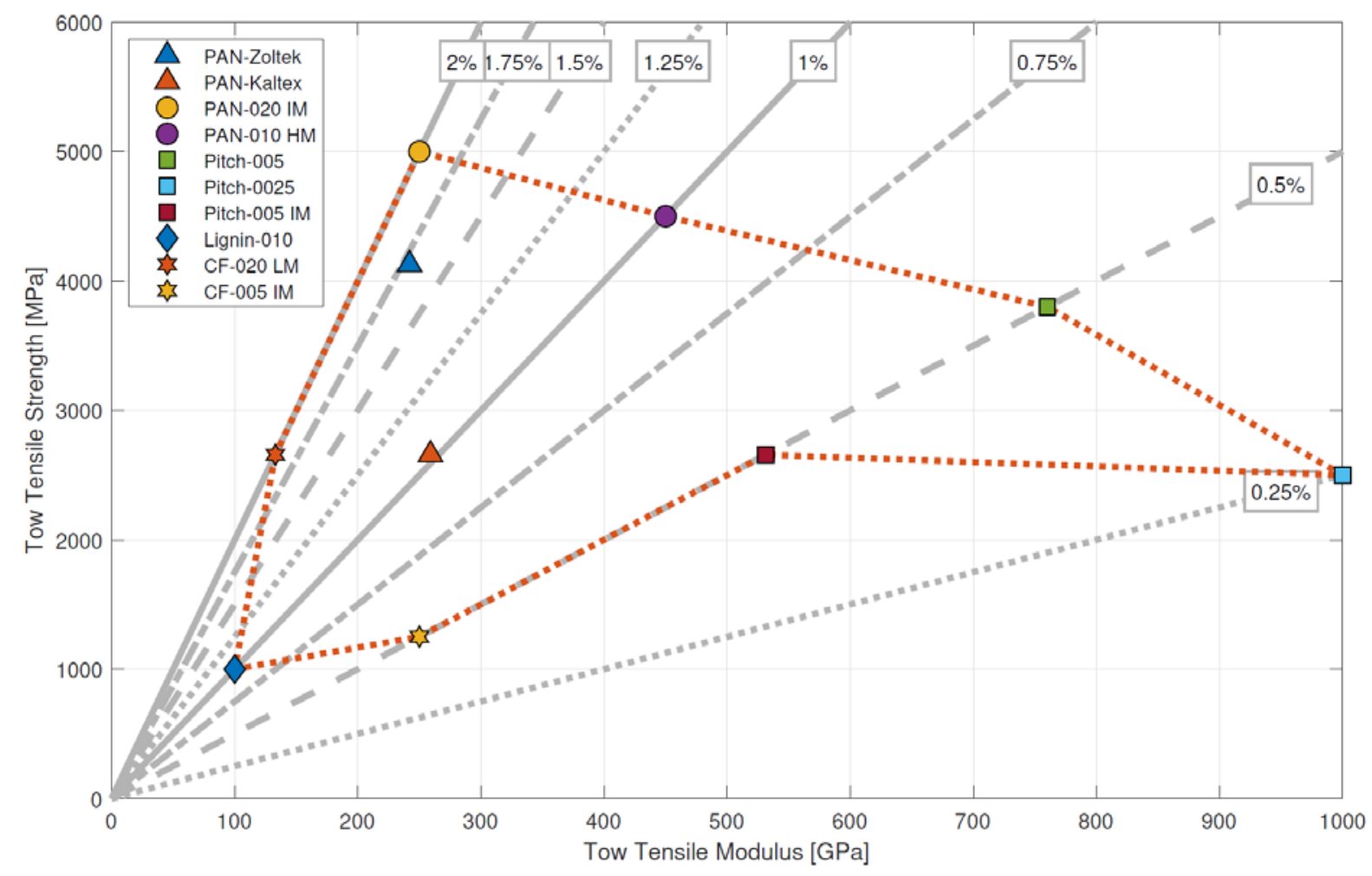

Figure 46. Carbon-fiber catalog tow properties representing a wide range of available commercial materials

\subsection{Subtask 5.B: Rotor Cost Optimization Studies}

The carbon-fiber characterization task results will be used to assess the various carbon systems in three relevant portions of a wind turbine blade: (1) the spar cap, (2) trailing-edge reinforcement, and (3) within the blade shell material. The spar-cap studies in this task will broaden the range of materials considered to identify the fundamental trade-offs among modulus, compressive strength, and failure strain to identify an optimal pathway for carbon-fiber material development. We will perform the studies looking at trailing-edge reinforcement and aeroelastic tailoring to determine the value of carbon-fiber composites in these less traditional applications to identify the properties of greatest significance. This work will be used to determine the value of various carbon-fiber systems that are not currently used by the wind energy industry and to identify pathways for further development of carbon-fiber materials, as summarized in Figure 47. 


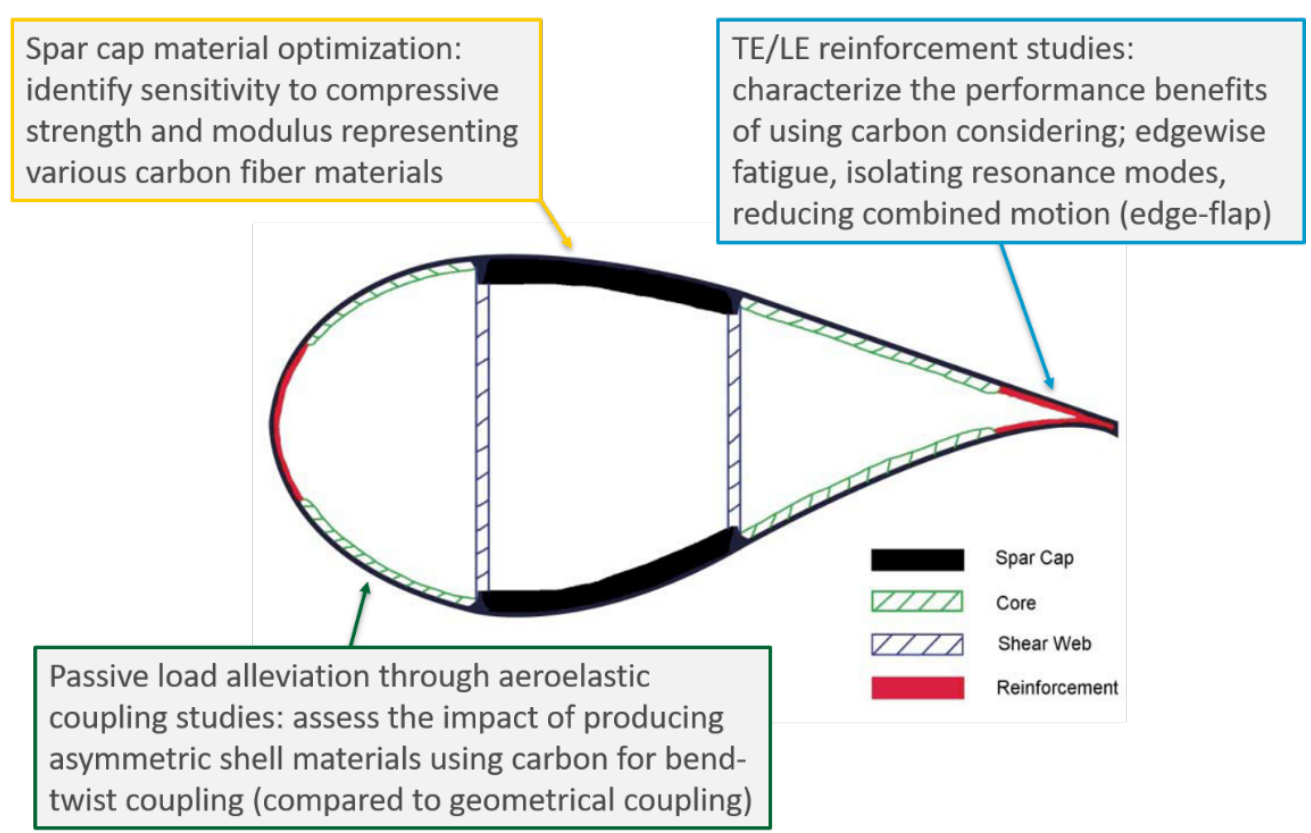

Figure 47. Design studies to be performed in Task 5 to assess the value of carbon fiber beyond the blade spar cap. Image credit: Optimized Carbon Fiber Composites in Wind Turbine Blade Design (Ennis et al. 2019) 


\section{Conclusions and Future Work}

The BAR program covered a variety of topics related to the development and deployment of very large, low-specific-power land-based wind turbines. Seventeen publications were released in Phase I of the BAR project. Overall, the project identified low-specific-power turbines as important to continued LCOE reductions for land-based turbines. Additionally, the benefits of low-specific-power turbines go beyond LCOE and are most valuable in regions with a high penetration of wind energy. Further, the project identified technical and logistical challenges to realizing these goals and proposed a set of viable solutions to address the challenges. We discovered that the highly flexible rail-transportable blade, downwind, and DAC concepts have the potential to enable continued reductions of LCOE. The most promising technologies that were identified in Phase I of the project will be further matured and derisked in Phase II of the BAR project.

Future work in Phase II will address the science and engineering challenges in designing systems identified in Phase I. Additionally, a refined techno-economic analysis will be conducted that includes some omissions from Phase I. Specifically, the BAR team will address the transportation and O\&M costs for the rail-transportable blades and the segmented blades. Improved cost models will be implemented for the DAC devices to enable more insight into the trade-offs involved with implementation. Experiments will be conducted to demonstrate new capabilities and gather important open-source data for model validation. 


\section{References}

Agarwala, Ranjeet, and Paul I. Ro. 2015. "Separated Pitch Control at Tip: Innovative Blade Design Explortions for Large MW Wind Turbine Blades.” Journal of Wind Energy 2015. http://dx.doi.org/10.1155/2015/895974.

Bir, Gunjit S. 2006. User's Guide to PreComp (pre-processor for computing composite blade properties). Golden, CO: National Renewable Energy Laboratory. NREL/TP-500-38929. https://www.nrel.gov/docs/fy06osti/38929.pdf.

BloombergNEF. 2018. "2H 2018 Wind Turbine Price Index.”

Bolinger, Mark, et al. 2020. "Opportunities for and challenges to further reductions in the "specific power" rating of wind turbines installed in the United States." Journal of Wind Engineering 45 (2): 351-368. https://doi.org/10.1177/0309524X19901012.

Bortolotti, Pietro, et al. 2019. A Detailed Wind Turbine Blade Cost Model. Golden, CO: National Renewable Energy Laboratory. NREL/TP-5000-73585. https://dx.doi.org/10.2172/1529217.

Bortolotti, Pietro, et al. 2020. Aeroacoustics Noise Model of OpenFAST. Golden, CO: National Renewable Energy Laboratory. NREL/TP-5000-75731. https://doi.org/10.2172/1660130.

Bortolotti, Pietro, et al. 2021a. "Validation Efforts of an Open-Source Aeroacoustics Model for Wind Turbines." Presented at the Ninth International Conference on Wind Turbine Noise May 18-21, 2021. https://www.nrel.gov/docs/fy21osti/79383.pdf.

Bortolotti, Pietro, Nick Johnson, Nikhar J. Abbas, Evan Anderson, Ernesto Camarena, and Joshua Paquette. 2021b. "Land-based wind turbines with flexible rail-transportable blades-Part 1: Conceptual design and aeroservoelastic performance." Journal of Wind Energy Science 6 (5): 1277-1290. https://doi.org/10.5194/wes-6-1277-2021.

Branlard, E., Pietro Bortolotti, Jason Jonkman. 2019. Aeroelastic Modelling Requirements for the BAR Project. Internal memo.

Burton, Tony, David Sharpe D, Nick Jenkins, et al. 2001. Wind Energy: Handbook. Chichester, New York: John Wiley.

Camarena, Ernesto, et al. Forthcoming. Part II: 3D FEM Design Optimization of the Rotor Blades. Albuquerque, NM: Sandia National Laboratories.

Carron, William Scott, and Pietro Bortolotti. 2020. "Innovative rail transport of a supersized land-based wind turbine blade." Journal of Physics: Conference Series 1618 (4) https://doi.org/10.1088/1742-6596/1618/4/042041. 
Chen, Hui, Wenbin Yu, and Mark Capellaro. 2010. "A critical assessment of computer tools for calculating composite wind turbine blade properties." Journal of Wind Energy 13 (6): 497-516. https://doi.org/10.1002/we.372.

Cole, Wesley, Will Frazier, Pual Donohoo-Vallett, et al. 2018. 2018 Standard Scenarios Report: A U.S. Electricity Sector Outlook. Golden, CO: National Renewable Energy Laboratory.

NREL/PR-6A20-72839. https://www.nrel.gov/docs/fy19osti/72839.pdf.

Ennis, Brandon, et al. 2019. Optimized Carbon Fiber Composites in Wind Turbine Blade Design. Albuquerque, NM: Sandia National Laboratories. SAND2019-14173.

https://doi.org/10.2172/1592956.

Ennis, Brandon, et al. Forthcoming. Optimized Carbon Fiber Composites in Wind Turbine Blade Design: Follow-on Studies. Albuquerque, NM: Sandia National Laboratories.

Feil, Roland, Nikhar Abbas, Pietro Bortolotti, Nick Johnson, and Ben Mertz. 2020a. "Distributed Aerodynamic Control using Active Trailing-Edge Flaps for Large Wind Turbines." Journal of Physics: Conference Series 1618 (4). https://doi.org/10.1088/1742-6596/1618/4/042026.

Feil, Roland, Tobias Pflumm, Pietro Bortolotti, and Marco Morandini. 2020b. "A cross-sectional aeroelastic analysis and structural optimization tool for slender composite structures." Journal of Composite Structures 253. https://doi.org/10.1016/j.compstruct.2020.112755.

Fingersh, Lee, M. Hand, and A. Laxon. 2006. Wind Turbine Design Cost and Scaling Model. Golden, CO: National Renewable Energy Laboratory. NREL/TP-500-40566. https://www.nrel.gov/docs/fy07osti/40566.pdf.

Garrett, Peter, Klaus Ronde. 2011a. Life Cycle Assessment of Electricity Production from a V802.0 MW Gridstreamer Wind Plant. Aarhus, Denmark: Vestas Wind Systems A/S.

Garrett, Peter, Klaus Ronde. 2011b. Life Cycle Assessment of Electricity Production from a V902.0 MW Gridstreamer Wind Plant. Aarhus, Denmark: Vestas Wind Systems A/S. https://www.vestas.com/content/dam/vestas-com/global/en/sustainability/reports-andratings/lcas/LCAV10020MW181215.pdf.coredownload.inline.pdf.

Gray, Justin, John Hwang, Joaquim Martins, Kenneth Moore, and Bret Naylor. 2019. "OpenMDAO: an open-source framework for multidisciplinary design, analysis, and optimization." Journal of Structural and Multidisciplinary Optimization 59 (4): 1075-1104. https://doi.org/10.1007/s00158-019-02211-z.

Hills, Richard, David Maniaci, and Jonathan Naughton. 2015. V\&V framework. Albuquerque, NM: Sandia National Laboratories. SAND 2015-7455. https://doi.org/10.2172/1214246.

Johnson, Nick, et al. 2019. Investigation of Innovative Rotor Concepts for the Big Adaptive Rotor Project. Golden, CO: National Renewable Energy Laboratory. NREL/TP-5000-73605. https://doi.org/10.2172/1563139. 
Lantz Eric, Owen Roberts, Jake Nunemaker, et al. 2019. Increasing Wind Turbine Tower Heights: Opportunities and Challenges. Golden, CO: National Renewable Energy Laboratory. NREL/TP-5000-73629. https://www.energy.gov/sites/default/files/2019/05/f63/73629.pdf.

Mendoza, Nicole, Roland Feil, Nick Johnson, and Benjamin Anderson. 2021. "Conceptual Designs of the Structure of Inflatable Blades for Enabling Larger Turbines." In AIAA Scitech 2021 Forum. p. 0815. https://doi.org/10.2514/6.2021-0815.

Miller, David, Daniel Samborsky, and Brandon Ennis. 2019. Mechanical Testing Summary: Optimized Carbon Fiber Composites in Wind Turbine Blade Design. Albuquerque, NM: Sandia National Laboratories. SAND2019-10780. https://doi.org/10.2172/1562792.

Moné Chris, Tyler Stehly, Ben Maples, and Edward Settle. 2015. 2014 Cost of Wind Energy Review. Golden, CO: National Renewable Energy Laboratory. NREL/TP-6A20-64281. https://www.nrel.gov/docs/fy16osti/64281.pdf.

Ragheb, Adam, and Michael Selig. 2011. "Multi-Element Airfoil Configurations for Wind Turbines." In 29th AIAA Applied aerodynamics conference, p. 3971. https://doi.org/10.2514/6.2011-3971.

Razdan, Priyanka, and Peter Garrett. 2015a. Life cycle assessment of electricity production from an onshore V100-2.0 MW Wind Plant. Aarhus, Denmark: Vestas Wind Systems A/S.

Razdan, Priyanka, and Peter Garrett. 2015b. Life cycle assessment of electricity production from an onshore V100-2.0 MW Wind Plant. Aarhus, Denmark: Vestas Wind Systems A/S.

Razdan, Priyanka, and Peter Garrett. 2018a. Life cycle assessment of electricity production from an onshore V116-2.0 MW Wind Plant. Aarhus, Denmark: Vestas Wind Systems A/S.

Razdan, Priyanka, and Peter Garrett. 2018b. Life cycle assessment of electricity production from an onshore V120-2.0 MW Wind Plant. Aarhus, Denmark: Vestas Wind Systems A/S.

Roth-Johnson, Perry, Richard Wirz, and Edward Lin. 2014. "Structural design of spars for 100-m biplane wind turbine blades." Journal of Renewable Energy 71: 133-155.

https://doi.org/10.1016/j.renene.2014.05.030.

Shaler, Kelsey, Emmanuel Branlard, and Andy Platt. 2020. OLAF User's Guide and Theory Manual. Golden, CO: National Renewable Energy Laboratory. NREL/TP-5000-75959. https://www.nrel.gov/docs/fy20osti/75959.pdf.

Sieros, Georgios, Panagiotis Chaviaropoulos, John D. Sørensen, Bernard H. Bulder, Peter Jamieson. 2012. "Upscaling wind turbines: theoretical and practical aspects and their impact on the cost of energy." Wind Energy 15(1): 3-17. https://doi.org/10.1002/we.527.

Smith, Kevin J., and Dayton A. Griffin. 2019. Supersized Wind Turbine Blade Study: R\&D Pathways for Supersized Wind Turbine Blades. Berkeley, CA: Lawrence Berkeley National Laboratory. https://doi.org/10.2172/1498695. 
Stehly, Tyler, Donna Heimiller, George Scott. 2017. 2016 Cost of Wind Energy Review. Golden, CO: National Renewable Energy Laboratory. NREL/TP-6A20-70363.

https://www.nrel.gov/docs/fy18osti/70363.pdf.

Stehly, Tyler, Philipp Beiter. 2019. 2018 Cost of Wind Energy Review. Golden, CO: National Renewable Energy Laboratory. NREL/TP-5000-74598.

https://www.nrel.gov/docs/fy20osti/74598.pdf.

U.S. Department of Energy. 2008. 20\% Wind Energy by 2030: Increasing Wind Energy's Contribution to U.S. Electricity Supply. Washington, DC.

https://www.nrel.gov/docs/fy08osti/41869.pdf.

U.S. Department of Energy. 2015. Wind Vision: A New Era for Wind Power in the United States. Washington, DC. https://www.energy.gov/sites/default/files/2015/03/f20/wv full_report.pdf.

Wang, Qi, Michael Sprague, Jason Jonkman, Nick Johnson, and Bonnie Jonkman. 2017. "BeamDyn: a high-fidelity wind turbine blade solver in the FAST modular framework." Journal of Wind Energy 20(8): 1439-1462. https://www.osti.gov/servlets/purl/1371521.

Wiser, Ryan, Mark Bolinger. 2019. 2018 Wind Technologies Market Report. Washington DC. https://escholarship.org/uc/item/2tc1819q.

Wiser, Ryan, Dev Millstein, Mark Bolinger, Seongeun Jeong, and Andrew Mills. 2021. "The hidden value of large-rotor, tall-tower wind turbines in the United States." Journal of Wind Engineering 45 (4): 857-871. https://doi.org/10.1177/0309524X20933949.

Wiser, Ryan, et al. 2012. "Recent developments in the levelized cost of energy from US wind power projects." Presentation, February 2012.

Wiser, Ryan, Zhenbin Yang, Maureen Hand, et al. 2011. Wind Energy. In: Edenhofer O, PichsMadruga R, Sokona Y, et al (eds) IPCC Special Report on Renewable Energy Sources and Climate Change Mitigation. Cambridge; New York: Cambridge University Press, pp. 535-607. https://www.ipcc.ch/site/assets/uploads/2018/03/Chapter-7-Wind-Energy-1.pdf. 


\section{Appendix A: Publications}

The following list shows all the conference papers, journal articles, and technical reports that were published during the Big Adaptive Rotor project.

Task 1:

1. Opportunities for and Challenges to Further Reductions in the "Specific Power" Rating of Wind Turbines Installed in the United States. https://doi.org/10.1177/0309524X19901012

2. The Hidden Value of Large-Rotor, Tall-Tower Wind turbines in the United States. https://doi.org/10.1177/0309524X20933949

Task 2:

1. Investigation of Innovative Rotor Concepts for the Big Adaptive Rotor Project. https://doi.org/10.2172/1563139

Task 3:

1. Supersized Wind Turbine Blade Study: R\&D Pathways for Supersized Wind Turbine Blades. https://doi.org/10.2172/1498695

Task 4:

1. A Detailed Wind Turbine Blade Cost Model. https://dx.doi.org/10.2172/1529217

2. Aeroacoustics Noise Model of OpenFAST. https://doi.org/10.2172/1660130

3. OLAF User's Guide and Theory Manual. https://www.nrel.gov/docs/fy20osti/75959.pdf

4. A Cross-Sectional Aeroelastic Analysis and Structural Optimization Tool for Slender composite Structures. https://doi.org/10.1016/j.compstruct.2020.112755

5. Distributed Aerodynamic Control using Active Trailing-Edge Flaps for Large Wind Turbines. https://doi.org/10.1088/1742-6596/1618/4/042026

6. Investigation of the Nacelle Blockage Effect for a Downwind Turbine. https://doi:10.1088/1742-6596/1618/6/062062

7. Innovative Rail Transport of a Supersized Land-Based Wind Turbine Blade. https://doi.org/10.1088/1742-6596/1618/4/042041

8. Investigation of Flutter for Large, Highly Flexible Wind Turbine Blades. https://doi.org/10.1088/1742-6596/1618/5/052078

9. Conceptual Designs of the Structure of Inflatable Blades for Enabling Larger Turbines. https://doi.org/10.2514/6.2021-0815

10. Land-Based Wind Turbines with Flexible Rail Transportable Blades - Part I: Conceptual Design and Aeroservoelastic Performance. https://doi.org/10.5194/wes-6-1277-2021

11. Part II: 3D FEM Design Optimization of the Rotor Blades (Forthcoming)

12. Preliminary Design of a Bi-Wing Wind Blade with Partial Span Pitch Control (Forthcoming)

Task 5:

1. Optimized Carbon Fiber Composites in Wind Turbine Blade Design. https://doi.org/10.2172/1592956 
2. Optimized Carbon Fiber Composites in Wind Turbine Blade Design: Follow-on Studies (Forthcoming) 